\title{
Computing $\mathcal{L}$-invariants for the symmetric square of an elliptic curve
}

by Daniel Delbourgo and Hamish Gilmore ${ }^{1}$

Department of Mathematics, University of Waikato, 3240 New Zealand.

email: daniel.delbourgo@waikato.ac.nz hjg10@students.waikato.ac.nz

2000 Mathematics Subject Classification: Primary 11R23; Secondary 11E95, 11G40

Abstract: Let $E$ be an elliptic curve over $\mathbb{Q}$, and $p \neq 2$ a prime of good ordinary reduction. The $p$-adic $L$-function for $\operatorname{Sym}^{2} E$ always vanishes at $s=1$, even though the complex $L$-function does not have a zero there. The $\mathcal{L}$-invariant itself appears on the right-hand side of the formula

$$
\left.\frac{\mathrm{d}}{\mathrm{d} s} \mathbf{L}_{p}\left(\operatorname{Sym}^{2} E, s\right)\right|_{s=1}=\mathcal{L}_{p}\left(\operatorname{Sym}^{2} E\right) \times\left(1-\alpha_{p}^{-2}\right)\left(1-p \alpha_{p}^{-2}\right) \times \frac{L_{\infty}\left(\mathrm{Sym}^{2} E, 1\right)}{(2 \pi i)^{-1} \Omega_{E}^{+} \Omega_{E}^{-}}
$$

where $X^{2}-a_{p}(E) X+p=\left(X-\alpha_{p}\right)\left(X-\beta_{p}\right)$ with $\alpha_{p} \in \mathbb{Z}_{p}^{\times}$.

We first devise a method to calculate $\mathcal{L}_{p}\left(\mathrm{Sym}^{2} E\right)$ effectively, then show it is non-trivial for all elliptic curves $E$ of conductor $N_{E} \leq 300$ with $4 \mid N_{E}$, and almost all ordinary primes $p<17$. Hence, in these cases at least, the order of the zero in $\mathbf{L}_{p}\left(\operatorname{Sym}^{2} E, s\right)$ at $s=1$ is exactly one.

\section{Contents}

1 Introduction 2

2 The Analytic Theory $\quad 5$

2.1 Petersson inner product identities for $f^{0} \ldots \ldots \ldots \ldots \ldots$

2.2 The $q$-expansion of the modular form $R_{m, e} \ldots \ldots \ldots \ldots \ldots$

2.3 Expressing $R_{m, e} \mid U_{p}^{2 m-1}$ in terms of a rational basis . . . . . . . . . . . 11

2.4 An explicit formula for $\mathcal{L}_{p}^{\text {an }}\left(\operatorname{Sym}^{2} E\right)$ modulo $p^{m}$, when $D(E, 1) \neq 0 \ldots \ldots \ldots$

2.5 Attempts at evaluating the moments $\int x^{j} \cdot \mathrm{d} \mu_{E}^{\text {imp }}$ for $j \neq 0$ ? . . . . . . . 14

3 The Basic Method $\quad 16$

3.1 An algorithm to compute the $\mathcal{L}$-invariant numerically $\ldots \ldots \ldots \ldots$

3.2 A general formula for $\mathbf{L}_{p}\left(\operatorname{Sym}^{2} E, 1\right)^{\prime}$ modulo $p^{m}$, even when $D(E, 1)=0 \ldots \ldots$

3.3 The connection with deformation theory . . . . . . . . . . . . 20

A Determining the set $S_{1}$, and the bad factors $H_{l}(X)$ with $l \in S_{1} \quad \mathbf{2 2}$

B Results for odd primes $p \leq 13$, in the range $11 \leq N_{E} \leq 300 \quad 23$

B.1 Tables of $\mathcal{L}$-invariants for elliptic curves $E$ with $D(E, 1) \neq 0 \ldots \ldots \ldots$

B.2 Tables of $\mathcal{L}$-invariants for elliptic curves $E$ with $D(E, 1)=0 \ldots \ldots \ldots$

\footnotetext{
${ }^{1}$ To form a part of this author's $\mathrm{PhD}$ thesis
} 


\section{Introduction}

Let $p$ be an odd prime. For a pure motive $M$ defined over $\mathbb{Q}$ of weight zero, there is a conjectural recipe to attach a $p$-adic $L$-function, $\mathbf{L}_{p}(M, s)$, provided its Galois representation is $p$-ordinary (see [6] for the precise details). The interpolated $p$-adic $L$-function should be related to its complex cousin, $L_{\infty}(M, s)$, at the critical point $s=0$, via the formula

$$
\mathbf{L}_{p}(M, 0)=\mathcal{E}_{p}(M, 0) \times \frac{L_{\infty}(M, 0)}{\Omega_{\infty}(M)} .
$$

Here $\mathcal{E}_{p}(M, s)$ is a product of certain Euler factors at $p$, and $\Omega_{\infty}(M)$ denotes the Deligne period.

Curiously, sometimes $\mathcal{E}_{p}(M, s)$ can vanish at $s=0$ even when $L_{\infty}(M, 0) \neq 0$, in which case we say that $M$ has an exceptional p-adic zero. Let us factorise out the trivial zero contribution into $\mathcal{E}_{p}(M, s)=\mathcal{E}_{p}^{\dagger}(M, s) \times \mathcal{E}_{p}^{\text {triv }}(M, s)$, where $\mathcal{E}_{p}^{\dagger}(M, 0) \neq 0$ and $\operatorname{order}_{s=0}\left(\mathcal{E}_{p}^{\text {triv }}(M, s)\right)=\mathbf{e}_{p}$. Greenberg [19] has associated an explicit invariant $\mathcal{L}_{p}^{\mathrm{Gr}}(M) \in \mathbb{Q}_{p}$, and he predicts that

$$
\left.\frac{\mathrm{d}^{\mathbf{e}_{p}} \mathbf{L}_{p}(M, s)}{\mathrm{d} s^{\mathbf{e}_{p}}}\right|_{s=0}=\mathcal{L}_{p}^{\mathrm{Gr}}(M) \times \mathcal{E}_{p}^{\dagger}(M, 0) \times \frac{L_{\infty}(M, 0)}{\Omega_{\infty}(M)} .
$$

One is naturally left to address the following problem.

Question. For a given motive $M$ as described above, and for an ordinary prime $p$ satisfying the exceptional zero condition, is Greenberg's $\mathcal{L}$-invariant term $\mathcal{L}_{p}^{\mathrm{Gr}}(M)$ non-zero?

For example, let $f$ be a primitive eigenform of weight $k \geq 2$, level $N$ and trivial nebentypus. Then the symmetric square motives $M=\operatorname{Sym}^{2}(f)(k-1)$ and $M=\operatorname{Sym}^{2}(f)(k)$ both exhibit exceptional $p$-adic zero phenomena at ordinary primes $p \nmid N$.

Over two decades ago, Coates and Greenberg suggested to the first-named author to compute $\mathcal{L}_{p}^{\mathrm{Gr}}\left(\operatorname{Sym}^{2}(f)(k-1)\right)$ as part of his $\mathrm{PhD}$, but he was unsuccessful and the project was shelved. Recently there has been a renewed interest in this topic $[2,22,30]$, in particular with the construction of global cohomology classes (an Euler system) for the motive $M(f \otimes f)$ in [27].

Under some standard assumptions, Hida has shown [26] that in a $\Lambda$-adic family of modular forms $\left\{\mathcal{F}_{k}\right\}_{k \in \mathcal{W}}$, the quantity $\mathcal{L}_{p}^{\mathrm{Gr}}\left(\operatorname{Sym}^{2}\left(\mathcal{F}_{k}\right)(k-1)\right)$ can vanish at only finitely many points in the weight-space $\mathcal{W}$. It therefore seems an appropriate time to revisit this open problem of non-vanishing for the symmetric square $\mathcal{L}$-invariant, albeit from a computational perspective.

Goal. To calculate $\mathcal{L}_{p}^{\mathrm{Gr}}\left(\operatorname{Sym}^{2}(f)(1)\right) \bmod p^{m}$ numerically when $f$ arises from an elliptic curve, and then check whether the associated $\mathcal{L}$-invariant is non-vanishing in a variety of examples.

Let $E$ be an elliptic curve over $\mathbb{Q}$, so that $E$ is necessarily modular by the work in $[4,35]$. Provided $\operatorname{Re}(s)>2$, the symmetric square $L$-function for $E$ is given by an Euler product

$$
L_{\infty}\left(\operatorname{Sym}^{2} E, s\right)=\left.\prod_{\text {primes } l} \operatorname{det}\left(1-\operatorname{Frob}_{l}^{-1} X \mid \operatorname{Sym}^{2} H_{\text {ét }}\left(\bar{E}, \mathbb{Q}_{q}(1)\right)^{I_{l}}\right)^{-1}\right|_{X=l^{-s}}
$$

where $q$ is any prime different from $l$, Frob $_{l}$ is an arithmetic Frobenius element, and $I_{l} \subset G_{\mathbb{Q}_{l}}$ denotes the inertia subgroup at $l$. If the prime number $l$ does not divide the $\mathbb{Q}$-conductor $N_{E}$ of the elliptic curve, then

$$
\operatorname{det}\left(1-\operatorname{Frob}_{l}^{-1} X \mid \operatorname{Sym}^{2} H_{\text {ét }}\left(\bar{E}, \mathbb{Q}_{q}(1)\right)^{I_{l}}\right)=\left(1-\alpha_{l}^{2} X\right)\left(1-\beta_{l}^{2} X\right)(1-l X)
$$

where $1-a_{l}(E) X+l X^{2}=\left(1-\alpha_{l} X\right)\left(1-\beta_{l} X\right)$ is the factorisation of the Hecke polynomial at $l$. Gelbart and Jacquet [17] showed that the function $L_{\infty}\left(\mathrm{Sym}^{2} E, s\right)$ has an analytic continuation to all $s \in \mathbb{C}$, and satisfies a functional equation linking the value at $s$ with the value at $3-s$. 
To describe Greenberg's $\mathcal{L}$-invariant term in detail, let us first fix an ordinary prime $p$. Consider the Galois representation $V=\operatorname{Sym}^{2}\left(H_{\text {ét }}\left(E, \mathbb{Q}_{p}(1)\right)^{*}\right) \cong \mathbb{Q}_{p} \otimes_{\mathbb{Z}_{p}} \operatorname{Sym}^{2}\left(\operatorname{Ta}_{p}(E)\right)$ where $\operatorname{Ta}_{p}(E)=\varliminf_{n} E_{p^{n}}$ is the $p$-adic Tate module of $E$. Viewed as a $G_{\mathbb{Q}_{p}}$-module, there is a filtration

$$
0=\mathrm{Fil}^{3} V \subset \mathrm{Fil}^{2} V \subset \mathrm{Fil}^{1} V \subset \mathrm{Fil}^{0} V=V
$$

where each quotient $\frac{\mathrm{Fil}^{i} V}{\mathrm{Fil}^{i+1} V}$ is isomorphic to $\mathbb{Q}_{p}(i)$ as an $I_{p}$-representation, for $i \in\{0,1,2\}$.

Let $\Sigma$ denote a finite set of primes containing $p$ and the primes of bad reduction for $E$. Associated to the $\operatorname{Gal}\left(\mathbb{Q}_{\Sigma} / \mathbb{Q}\right)$-representation $V$ in $[3]$ are the Bloch-Kato Selmer groups

$$
H_{f,\{p\}}^{1}(\mathbb{Q}, V):=\operatorname{Ker}\left(H^{1}\left(\operatorname{Gal}\left(\mathbb{Q}_{\Sigma} / \mathbb{Q}\right), V\right) \stackrel{\oplus \operatorname{res}_{l}}{\longrightarrow} \bigoplus_{l \in \Sigma, l \neq p} H^{1}\left(I_{l}, V\right)\right)
$$

and

$$
H_{f}^{1}(\mathbb{Q}, V):=\operatorname{Ker}\left(H_{f,\{p\}}^{1}(\mathbb{Q}, V) \stackrel{\operatorname{res}_{p}}{\longrightarrow} \frac{H^{1}\left(G_{\mathbb{Q}_{p}}, V\right)}{H_{f}^{1}\left(G_{\mathbb{Q}_{p}}, V\right)}\right)
$$

where $H_{f}^{1}\left(G_{\mathbb{Q}_{p}}, V\right)$ denotes the kernel of the mapping from $H^{1}\left(G_{\mathbb{Q}_{p}}, V\right)$ to $H^{1}\left(G_{\mathbb{Q}_{p}}, V \otimes B_{\text {cris }}\right)$. Flach et al. $[16,35,12]$ have shown $H_{f}^{1}(\mathbb{Q}, V)=\{0\}$, which implies that $\operatorname{dim}_{\mathbb{Q}_{p}} H_{f,\{p\}}^{1}(\mathbb{Q}, V)=1$. Let us fix a generator $\eta$ of this line, so that $H_{f,\{p\}}^{1}(\mathbb{Q}, V)=\mathbb{Q}_{p} \cdot \eta$.

We now explain how to choose coordinates. Observe first that $H^{1}\left(G_{\mathbb{Q}_{p}}, V\right)=H^{1}\left(G_{\mathbb{Q}_{p}}, \mathrm{Fil}^{1} V\right)$, an assertion that can be checked from the local formula

$$
\operatorname{dim}_{\mathbb{Q}_{p}} H^{1}\left(G_{\mathbb{Q}_{p}}, W\right)=\operatorname{dim}_{\mathbb{Q}_{p}}\left(W \otimes B_{\text {cris }}\right){ }^{G_{\mathbb{Q}_{p}}}+\operatorname{dim}_{\mathbb{Q}_{p}} H^{0}\left(G_{\mathbb{Q}_{p}}, W\right)+\operatorname{dim}_{\mathbb{Q}_{p}} H^{0}\left(G_{\mathbb{Q}_{p}}, W^{*}(1)\right)
$$

which yields the value $3+0+0$ if $W=V$, and $2+0+1$ if $W=$ Fil $^{1} V$. Applying Kummer theory, there is a natural identification $H^{1}\left(G_{\mathbb{Q}_{p}}, \mathbb{Q}_{p}(1)\right) \cong \mathbb{Q}_{p} \otimes_{\mathbb{Z}_{p}}\left(\lim _{n} \mathbb{Q}_{p}^{\times} / \mathbb{Q}_{p}^{\times} p^{n}\right)$, from which one obtains the homomorphism

$\mathfrak{q}: H^{1}\left(G_{\mathbb{Q}_{p}}, V\right)=H^{1}\left(G_{\mathbb{Q}_{p}}\right.$, Fil $\left.^{1} V\right) \stackrel{\operatorname{mod~Fii}}{\longrightarrow} H^{1}\left(G_{\mathbb{Q}_{p}}\right.$, Fil $^{1} V /$ Fil $\left.^{2} V\right) \stackrel{\sim}{\longrightarrow} \mathbb{Q}_{p} \otimes_{\mathbb{Z}_{p}}\left({\underset{\leftarrow}{n}}_{\lim _{p}} \mathbb{Q}_{p}^{\times} / \mathbb{Q}_{p}^{\times} p^{n}\right)$.

Furthermore, on the right-hand target space there is an isomorphism

$$
\mathbb{Q}_{p} \otimes_{\mathbb{Z}_{p}}\left({\underset{\leftarrow}{n}}_{\lim _{n}} \mathbb{Q}_{p}^{\times} / \mathbb{Q}_{p}^{\times p^{n}}\right) \stackrel{\sim}{\longrightarrow} \mathbb{Q}_{p} \times \mathbb{Q}_{p} \quad \text { sending } q \mapsto\left(\log _{p}(q), \operatorname{ord}_{p}(q)\right),
$$

where $\log _{p}: \mathbb{Q}_{p}^{\times} \rightarrow \mathbb{Q}_{p}$ denotes Iwasawa's logarithm map, normalised so that $\log _{p}(p)=0$.

Definition 1.1. The arithmetic $\mathcal{L}$-invariant is defined to be the slope of $\mathfrak{q} \circ \operatorname{res}_{p}(\eta)$ inside the vector space $H^{1}\left(G_{\mathbb{Q}_{p}}, \mathrm{Fil}^{1} V / \mathrm{Fil}^{2} V\right) \cong \mathbb{Q}_{p} \times \mathbb{Q}_{p}$, i.e.

$$
\mathcal{L}_{p}^{\mathrm{Gr}}\left(\operatorname{Sym}^{2} E\right):=\frac{\log _{p}\left(\mathfrak{q}\left(\operatorname{res}_{p}(\eta)\right)\right)}{\operatorname{ord}_{p}\left(\mathfrak{q}\left(\operatorname{res}_{p}(\eta)\right)\right)}
$$

which is clearly independent of the choice of generator $\eta$ for the $\mathbb{Q}_{p}$-line $H_{f,\{p\}}^{1}(\mathbb{Q}, V)$.

In fact, there is a more analytic way to introduce the $\mathcal{L}$-invariant if we work with the $p$-adic $L$-function directly. Depending on the reduction type of the curve, $p$-adic $L$-functions which interpolate Dirichlet twists of $\operatorname{Sym}^{2}\left(h^{1}(E)\right)(1)$ have been constructed in $[7,9,11,21,24,31]$. Now if $E$ has good ordinary reduction at $p$, there exists $\mathcal{F}(X) \in X \cdot \mathbb{Z}_{p} \llbracket X \rrbracket \otimes \mathbb{Q}$ such that

$$
\mathcal{F}(\chi(1+p)-1)=\frac{\tau(\bar{\chi})}{\alpha_{p}^{2 m_{\chi}}} \times \frac{L_{\infty}\left(\mathrm{Sym}^{2} E \otimes \chi, 1\right)}{(2 \pi i)^{-1} \Omega_{E}^{+} \Omega_{E}^{-}}
$$


at all non-trivial characters $\chi$ of conductor $\mathfrak{f}_{\chi}=p^{m_{\chi}}>1$ satisfying $\left.\chi\right|_{\mathbb{F}_{p}^{\times}}=\mathbf{1}$, while $\mathcal{F}(0)=0$. Here $\alpha_{p}$ is the $p$-adic unit root of $X^{2}-a_{p}(E) X+p$, secondly $\tau(\bar{\chi})$ denotes a Gauss sum for $\chi^{-1}$, and lastly $\Omega_{E}^{ \pm}$are real/imaginary periods associated to a minimal Weierstrass equation for $E_{/ \mathbb{Z}}$.

Definition 1.2. We write $\mathbf{L}_{p}\left(\operatorname{Sym}^{2} E,-\right): \mathbb{Z}_{p} \rightarrow \mathbb{Q}_{p}$ for the Mazur-Mellin transform

$$
\mathbf{L}_{p}\left(\operatorname{Sym}^{2} E, s\right):=\mathcal{F}\left((1+p)^{s-1}-1\right),
$$

so that $\mathbf{L}_{p}\left(\operatorname{Sym}^{2} E, s\right)$ has an exceptional zero at $s=1$.

In the late 1980s, Coates and Greenberg made the following prediction about its first derivative.

Conjecture 1.3. If $E$ has good ordinary reduction at $p$, the $\mathcal{L}$-invariant given by the ratio

$$
\mathcal{L}_{p}^{\mathrm{an}}\left(\operatorname{Sym}^{2} E\right):=\left.\frac{\mathrm{d}}{\mathrm{d} s} \mathbf{L}_{p}\left(\operatorname{Sym}^{2} E, s\right)\right|_{s=1} \times\left(\left(1-\alpha_{p}^{-2}\right)\left(1-p \alpha_{p}^{-2}\right) \times \frac{L_{\infty}\left(\operatorname{Sym}^{2} E, 1\right)}{(2 \pi i)^{-1} \Omega_{E}^{+} \Omega_{E}^{-}}\right)^{-1}
$$

is a non-zero $p$-adic number, so in particular

$$
\operatorname{order}_{s=1}\left(\mathbf{L}_{p}\left(\operatorname{Sym}^{2} E, s\right)\right)=1 .
$$

As will be discussed at length in $\S 3.3$, in most situations the work of Citro, Dasgupta and Hida $[5,10,26]$ implies that $\mathcal{L}_{p}^{\mathrm{an}}\left(\operatorname{Sym}^{2} E\right)=\mathcal{L}_{p}^{\mathrm{Gr}}\left(\operatorname{Sym}^{2} E\right)$, so we may shift between these two definitions as appropriate. In particular, the non-vanishing of the analytic $\mathcal{L}$-invariant means that the line $H_{f,\{p\}}^{1}(\mathbb{Q}, V)$ has a non-trivial slope inside $\mathbb{Q}_{p} \otimes_{\mathbb{Z}_{p}}\left(\varliminf_{n} \mathbb{Q}_{p}^{\times} / \mathbb{Q}_{p}^{\times p^{n}}\right) \cong \mathbb{Q}_{p} \times \mathbb{Q}_{p}$.

Remarks: (a) If $E$ has complex multiplication, then a result of Ferrero and Greenberg [14] implies that $\mathcal{L}_{p}^{\text {an }}\left(\operatorname{Sym}^{2} E\right)=\log _{p}\left(\alpha_{p}^{-2}\right)$; therefore in the CM case, Conjecture 1.3 is at least known to be true.

(b) If $E$ has split multiplicative reduction at $p$, under certain restrictions Rosso [30] recently proved $\mathcal{L}_{p}^{\text {an }}\left(\operatorname{Sym}^{2} E\right)=\log _{p}\left(q_{E}\right) / \operatorname{ord}_{p}\left(q_{E}\right)$ where $q_{E}$ is the Tate period of the rigid analytic curve; moreover $\log _{p}\left(q_{E}\right) \neq 0$ by [1, Theorem 3], so Conjecture 1.3 holds in this situation too.

(c) We should also point out that in the case where $E$ has split multiplicative reduction at $p$, the Tate period $q_{E}$ is a universal norm for the $\mathbb{Z}_{p}$-extension $F_{\infty} / \mathbb{Q}_{p}$ cut out by

$$
\operatorname{Im}\left(H^{1}\left(G_{\mathbb{Q}_{p}}, \operatorname{Sym}^{2} \operatorname{Ta}_{p}(E)\right) \stackrel{\operatorname{mod~Fil}}{\longrightarrow} H^{1}\left(G_{\mathbb{Q}_{p}}, \mathbb{Z}_{p}\right)\right)
$$

inside $H^{1}\left(G_{\mathbb{Q}_{p}}, \mathbb{Z}_{p}\right)=\operatorname{Hom}\left(G_{\mathbb{Q}_{p}}, \mathbb{Z}_{p}\right) \cong \mathbb{Z}_{p}^{2}$. Under the Tate local pairing

$$
H^{1}\left(G_{\mathbb{Q}_{p}}, \mathbb{Q}_{p}(1)\right) \times H^{1}\left(G_{\mathbb{Q}_{p}}, \mathbb{Q}_{p}\right) \rightarrow \mathbb{Q}_{p},
$$

the line $\mathfrak{q} \circ \operatorname{res}_{p}\left(H_{f,\{p\}}^{1}(\mathbb{Q}, V)\right)$ will then be orthogonal to the subspace $\operatorname{Hom}\left(\operatorname{Gal}\left(F_{\infty} / \mathbb{Q}_{p}\right), \mathbb{Q}_{p}\right)$. Applying exactly the same reasoning as $[19, \mathrm{p} 154]$, it follows that the $\operatorname{slopes} \log _{p}\left(q_{E}\right) / \operatorname{ord}_{p}\left(q_{E}\right)$ and $\log _{p}\left(\mathfrak{q}\left(\operatorname{res}_{p}(\eta)\right)\right) / \operatorname{ord}_{p}\left(\mathfrak{q}\left(\operatorname{res}_{p}(\eta)\right)\right)$ are actually equal. ${ }^{2}$

(d) Using efficient methods to compute overconvergent modular symbols, Dummit et al [13, §7.2] have computed $\mathcal{L}_{p}^{\text {an }}\left(\operatorname{Sym}^{2} E\right)$ for $(E, p)=\left(X_{0}(11), 11\right)$, and for $p=5$ and $E \in\{15 a 1,19 a 1,95 a 1\}$ (here we employ Cremona's elliptic curve labelling from [8]).

By devising algorithms to compute $\mathbf{L}_{p}\left(\mathrm{Sym}^{2} E, 1\right)^{\prime}$ and $\mathcal{L}_{p}^{\mathrm{an}}\left(\mathrm{Sym}^{2} E\right)$ numerically to a reasonable accuracy, and then implementing them into SAGE, we have established the following result.

\footnotetext{
${ }^{2}$ In the case of split multiplicative reduction the $\mathcal{L}$-invariant for $\operatorname{Sym}^{2} E$ is the same as the $\mathcal{L}$-invariant for $E$, and it is further conjectured (by Greenberg) that the $\mathcal{L}$-invariants for $\mathrm{Sym}^{m} E$ should be independent of $m>0$.
} 
Theorem 1.4. Let $E$ be an elliptic curve over $\mathbb{Q}$ of conductor $N_{E} \leq 300$, with 4 dividing $N_{E}$.

(i) If $p \in\{3,5,7\}$ is a prime of good ordinary reduction for $E$ then Conjecture 1.3 is true,

$$
\text { i.e. } \quad \mathcal{L}_{p}^{\text {an }}\left(\operatorname{Sym}^{2} E\right) \neq 0 \quad \text { and } \operatorname{order}_{s=1}\left(\mathbf{L}_{p}\left(\operatorname{Sym}^{2} E, s\right)\right)=1 .
$$

(ii) If $p=11$ is a prime of good ordinary reduction for $E$ then Conjecture 1.3 is true, with the possible exception of the following four elliptic curves:

116a1, 124b1, 200a1, 296a1.

(iii) If $p=13$ is a prime of good ordinary reduction for $E$ then Conjecture 1.3 is true, with the possible exception of the following six elliptic curves:

140a1, 200b1, 232b1, 244a1, 272b1, 280a1.

Here is a brief plan of the paper.

Sections 2.1-2.5 explain the theory behind our method. We first derive a technical result about the Petersson inner product in $\S 2.1$. In the next two sections, we relate the moments of the $p$-adic measure interpolating $\operatorname{Sym}^{2} E(1)$ to a specific inner product involving the weight two newform $f_{E}$ obtained from $E$ by modularity, and an auxiliary weight two form " $R \mid U_{p}^{2 m-1}$ " which is independent of the elliptic curve. Then in $\S 2.4$, we use our identity from $\S 2.1$ to obtain an expression for $\mathbf{L}_{p}\left(\mathrm{Sym}^{2} E, 1\right)^{\prime} \bmod p^{m}$, thereby yielding an approximation to $\mathcal{L}_{p}^{\text {an }}\left(\mathrm{Sym}^{2} E\right)$. Lastly in $\S 2.5$, we discuss how one might increase both the speed and the accuracy of our computations by instead evaluating Tate-twists for the $p$-adic measure, although there remain significant technical hurdles to overcome if one adopts this approach.

Sections 3.1-3.2 contain an implementation of our algorithms. We chose $N_{E} \leq 300$ and $p \leq 13$ as our bounds to determine whether or not $\mathbf{L}_{p}\left(\mathrm{Sym}^{2} E, 1\right)^{\prime}$ was non-zero, and thence to tabulate the $\mathcal{L}$-invariants to a decent accuracy - see Appendix B for the full numerical results. It took ten months to run our programs within these limited ranges, on a single core of an Intel i5-2400. The $4+6=10$ missing pairs $(E, p)$ in Theorem 1.4(ii)-(iii) occurred because the run-time required to show that $\mathbf{L}_{p}\left(\operatorname{Sym}^{2} E, 1\right)^{\prime} \neq 0$ for these specimens was too slow.

Finally in $\S 3.3$, we discuss how the work of Hida, Citro and Dasgupta $[26,5,10]$ combined with Theorem 1.4 implies the non-vanishing for the derivative of the Hecke eigenvalue $a_{p}\left(\mathcal{F}_{k}\right)$ at weight $k=2$, where $\mathcal{F}$ denotes the $p$-ordinary family lifting $f_{E}$.

Acknowledgements. The first author warmly thanks John Coates and Ralph Greenberg for originally suggesting this problem. Both authors are also grateful to Antonio Lei, Max Flander and Denis Benois for their helpful suggestions on computing symmetric square $\mathcal{L}$-invariants. They are also grateful to the referee for their insights, in particular the theoretical approach suggested in Section 2.5 (although we have been unable to implement this approach in practice). The second named author is financially supported by a University of Waikato PhD scholarship.

\section{The Analytic Theory}

For an integer $N>1$, we write $\mathcal{M}_{2}\left(\Gamma_{0}(N)\right)$ for the $\mathbb{C}$-vector space of modular forms of weight two, level $N$ and trivial nebentypus. We also denote by $\mathcal{S}_{2}\left(\Gamma_{0}(N)\right)$ the subspace of cusp forms. Throughout one normalises the Petersson inner product by

$$
\langle g, h\rangle_{N}:=\int_{\Gamma_{0}(N) \backslash \mathfrak{H}} \overline{g(z)} h(z) \cdot \mathrm{d} x \mathrm{~d} y \quad \text { for all } g \in \mathcal{S}_{2}\left(\Gamma_{0}(N)\right) \text { and } h \in \mathcal{M}_{2}\left(\Gamma_{0}(N)\right) .
$$


Let $f_{E} \in \mathcal{S}_{2}^{\text {new }}\left(\Gamma_{0}\left(N_{E}\right)\right)$ denote the primitive form associated to the modular elliptic curve $E$. Without loss of generality, we assume that

- the conductor $N_{E}$ of the newform $f_{E}$ is divisible by 4 .

Because $L_{\infty}\left(\operatorname{Sym}^{2} E, s\right)$ is invariant under taking quadratic twists, one can always ensure that the above holds by replacing $E$ with its twist by the unique character of conductor 4 (if necessary). We also modify the quantities in Conjecture 1.3, as follows:

- we swap the motivic period $(2 \pi i)^{-1} \Omega_{E}^{+} \Omega_{E}^{-}$with the automorphic period $\pi\left\langle f_{E}, f_{E}\right\rangle_{N_{E}}$;

- we shall exchange the primitive symmetric square $L$-function $L_{\infty}\left(\operatorname{Sym}^{2} E \otimes \chi, s\right)$ with its imprimitive version

$$
D(E, \chi, s):=L_{\mathfrak{f}_{\chi} N_{E}}\left(\chi^{2}, 2 s-2\right) \times \sum_{n=1}^{\infty} \frac{\chi(n) a_{n^{2}}(E)}{n^{s}} ;
$$

- we replace the $p$-adic $L$-function with $\mathbf{L}_{p}^{\text {imp }}\left(\operatorname{Sym}^{2} E, s\right):=\mathcal{F}^{\text {imp }}\left((1+p)^{s-1}-1\right)$, where

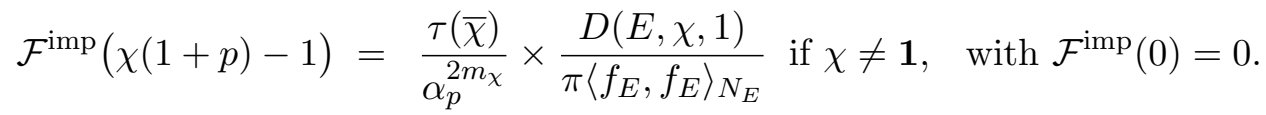

Providing the imprimitive $L$-function is non-vanishing at $s=1$ so that $D(E, 1) \neq 0$, the $\mathcal{L}$-invariant may be equivalently rewritten as

$$
\mathcal{L}_{p}^{\mathrm{an}}\left(\operatorname{Sym}^{2} E\right):=\left.\frac{\mathrm{d}}{\mathrm{d} s} \mathbf{L}_{p}^{\mathrm{imp}}\left(\operatorname{Sym}^{2} E, s\right)\right|_{s=1} \times\left(\left(1-\alpha_{p}^{-2}\right)\left(1-p \alpha_{p}^{-2}\right) \times \frac{D(E, 1)}{\pi\left\langle f_{E}, f_{E}\right\rangle_{N_{E}}}\right)^{-1} .
$$

The right-hand bracketed term in Equation (1) is reasonably straightforward to evaluate.

Lemma 2.1. Assume $E$ has minimal conductor amongst its quadratic twists. If the geometric conductor of $\operatorname{Sym}^{2}\left(h^{1}(E)\right)$ is denoted by $C_{\mathrm{Sym}^{2} E} \in \mathbb{N}^{2}$, then one has the formula

$$
\frac{D(E, 1)}{\pi\left\langle f_{E}, f_{E}\right\rangle_{N_{E}}}=\frac{4 \cdot \sqrt{C_{\mathrm{Sym}^{2} E}}}{N_{E}} \times \prod_{l \in S_{1}} \frac{H_{l}\left(l^{-1}\right)}{H_{l}\left(l^{-2}\right)}
$$

where $L_{\infty}\left(\operatorname{Sym}^{2} E \otimes \chi, s\right)=D(E, \chi, s) \times \prod_{l \in S_{1}} H_{l}\left(\chi(l) l^{-s}\right)^{-1}$ for a finite set of bad primes $S_{1}$.

Proof. If we define $\Lambda_{\infty}\left(\operatorname{Sym}^{2} E, s\right):=\left(C_{\mathrm{Sym}^{2} E}\right)^{s / 2} \cdot \pi^{-s / 2} \Gamma(s / 2)(2 \pi)^{-s} \Gamma(s) \times L_{\infty}\left(\operatorname{Sym}^{2} E, s\right)$, then the functional equation [7, Thm 2.2] for this completed $L$-function states that

$$
\Lambda_{\infty}\left(\operatorname{Sym}^{2} E, s\right)=\Lambda_{\infty}\left(\operatorname{Sym}^{2} E, 3-s\right) .
$$

Combining the above equation at $s=2$ with the formula $D(E, 2)=\frac{8 \pi^{3}}{N_{E}} \times\left\langle f_{E}, f_{E}\right\rangle_{N_{E}}$ for the imprimitive symmetric square $L$-function in [15, Equation (5)], the result follows easily.

To calculate $\mathcal{L}_{p}^{\mathrm{an}}\left(\mathrm{Sym}^{2} E\right)$ numerically, we must therefore evaluate $\frac{\mathrm{d}}{\mathrm{d} s} \mathbf{L}_{p}^{\mathrm{imp}}\left(\mathrm{Sym}^{2} E, s\right)$ at $s=1$ to a reasonable accuracy. If $\mu_{E}^{\text {imp }} \in \operatorname{Meas}\left(\mathbb{Z}_{p}^{\times}, \mathbb{Q}_{p}\right)$ is the $p$-bounded measure corresponding to the power series $\mathcal{F}^{\operatorname{imp}}(X) \in X \cdot \mathbb{Z}_{p} \llbracket X \rrbracket[1 / p]$, then

$$
\mathbf{L}_{p}^{\mathrm{imp}}\left(\operatorname{Sym}^{2} E, s\right)=\int_{x \in \mathbb{Z}_{p}^{\times}}\left\langle x>_{p}^{s-1} \cdot \mathrm{d} \mu_{E}^{\mathrm{imp}}(x) \text { for every } s \in \mathbb{Z}_{p},\right.
$$


where $<->_{p}$ : $\mathbb{Z}_{p}^{\times} \rightarrow 1+p \mathbb{Z}_{p}$ denotes the projection to the principal local units. It follows that $\left.\frac{\mathrm{d}}{\mathrm{d} s} \mathbf{L}_{p}^{\mathrm{imp}}\left(\operatorname{Sym}^{2} E, s\right)\right|_{s=1}=\int_{x \in \mathbb{Z}_{p}^{\times}} \log _{p}<x>_{p} \cdot \mathrm{d} \mu_{E}^{\mathrm{imp}}(x) \approx \sum_{e \in\left(\mathbb{Z} / p^{m} \mathbb{Z}\right)^{\times}} \log _{p}<e>_{p} \times \mu_{E}^{\mathrm{imp}}\left(e+p^{m} \mathbb{Z}_{p}\right)$ upon using a Riemann sum approximation for the covering $\mathbb{Z}_{p}^{\times}=\bigsqcup_{e}\left(e+p^{m} \mathbb{Z}_{p}\right)$.

Question. Given a class $e \in\left(\mathbb{Z} / p^{m} \mathbb{Z}\right)^{\times}$, how do we calculate each $\mu_{E}^{\mathrm{imp}}\left(e+p^{m} \mathbb{Z}_{p}\right)$ efficiently? It is well known $[7,9,31]$ the moments $\mu_{E}^{\mathrm{imp}}\left(e+p^{m} \mathbb{Z}_{p}\right)$ can be written as an inner product of

$$
f^{0}(z):=\left(f_{E}(z)-\beta_{p} f_{E}(p z)\right) \mid\left(\begin{array}{cc}
0 & -1 \\
p N_{E} & 0
\end{array}\right) \in \mathcal{S}_{2}\left(\Gamma_{0}\left(p N_{E}\right)\right)
$$

with a certain modular form $R_{m, e} \in \mathcal{M}_{2}\left(\Gamma_{0}\left(p N_{E}\right)\right)$, whose Fourier coefficients are $p$-integral. The integrality of $\mu_{E}^{\mathrm{imp}}(-)$ is then controlled by that of $\frac{\left\langle f^{0}, R_{m, e}\right\rangle_{p N_{E}}}{\left\langle f_{E}, f_{E}\right\rangle}$ for varying $m$ and $e$.

\subsection{Petersson inner product identities for $f^{0}$}

Recall that the functional equation for the completed Hasse-Weil $L$-function, $\Lambda_{\infty}(E, s)$, has the form $\Lambda_{\infty}(E, 2-s)=w_{E} \times \Lambda_{\infty}(E, s)$ where $w_{E} \in\{ \pm 1\}$ denotes the root number for $E$ over $\mathbb{Q}$. In terms of the associated newform,

$$
f_{E} \mid W\left(N_{E}\right)=-w_{E} \cdot f_{E} \quad \text { under the action of } W\left(N_{E}\right)=\left(\begin{array}{cc}
0 & -1 \\
N_{E} & 0
\end{array}\right) .
$$

Let $h(z)$ denote a weight 2 holomorphic modular form of level $p N_{E}$, and with trivial character. Our goal here is to derive the following technical result, which we repeatedly make use of later.

Lemma 2.2. (i) If $\mathbb{C} \cdot h(z) \cap\left(\mathbb{C} \cdot f_{E}(z) \oplus \mathbb{C} \cdot f_{E}(p z)\right)=\{0\}$, then $\left\langle f^{0}, h\right\rangle_{p N_{E}}=0$;

(ii) If $w_{E} \in\{ \pm 1\}$ is the root number for $E_{/ \mathbb{Q}}$, then $\left\langle f^{0}, f_{E}(z)\right\rangle_{p N_{E}}=-w_{E} \cdot \frac{\alpha_{p}^{2}-1}{\alpha_{p}} \times\left\langle f_{E}, f_{E}\right\rangle_{N_{E}}$; (iii) Replacing $f_{E}(z)$ with $f_{E}(p z)$, one instead has $\left\langle f^{0}, f_{E}(p z)\right\rangle_{p N_{E}}=-w_{E} \cdot \frac{\alpha_{p}^{2}-1}{\alpha_{p}^{2}} \times\left\langle f_{E}, f_{E}\right\rangle_{N_{E}}$.

Proof. The $f_{E}$-isotypic part of $\mathcal{M}_{2}\left(\Gamma_{0}\left(p N_{E}\right)\right)$ consists of the subspace $\mathbb{C} \cdot f_{E}(z) \oplus \mathbb{C} \cdot f_{E}(p z)$. Without loss of generality, assume $h(z)$ is an eigenform for the Hecke algebra at level $p N_{E}$. Then by multiplicity one, we can pick a prime $l \nmid p N_{E}$ such that $a_{l}\left(f_{E}\right) \neq a_{l}(h)$; consequently

$$
a_{l}\left(f_{E}\right) \times\left\langle f^{0}, h\right\rangle_{p N_{E}}=\left\langle f^{0} \mid T_{l}^{*}, h\right\rangle_{p N_{E}}=\left\langle f^{0}, h \mid T_{l}\right\rangle_{p N_{E}}=a_{l}(h) \times\left\langle f^{0}, h\right\rangle_{p N_{E}}
$$

in which case $\left\langle f^{0}, h\right\rangle_{p N_{E}}=0$, so part (i) is true.

To establish statement (ii), let us first introduce the $p$-stabilisation

$$
f_{0}(z):=f_{E}(z)-\beta_{p} f_{E}(p z)=\alpha_{p}^{-1} \cdot f_{E} \mid\left(U_{p}-\beta_{p} I_{2}\right) \in \mathcal{S}_{2}\left(\Gamma_{0}\left(p N_{E}\right)\right) .
$$

This cusp form $f_{0}$ is related to $f^{0}$ through the formula

$$
f^{0}(z)=f_{E}^{\rho}\left|W\left(p N_{E}\right)-\alpha_{p} p^{-1} f_{E}^{\rho}\right| W\left(N_{E}\right)=f_{0}^{\rho} \mid W\left(p N_{E}\right),
$$


where the involution $(-)^{\rho}$ above sends each $h(z)=\sum_{n \geq 1} h_{n} e^{2 \pi i n z}$ to $h^{\rho}(z)=\sum_{n \geq 1} \overline{h_{n}} e^{2 \pi i n z}$. Now using Equation (3) and observing that $f_{E}^{\rho}=f_{E}$, one obtains the equalities

$$
\begin{aligned}
&\left\langle f^{0}, f_{E}\right\rangle_{p N_{E}}=\left\langle f_{E}^{\rho} \mid W\left(p N_{E}\right), f_{E}\right\rangle_{p N_{E}}-\overline{\alpha_{p} p^{-1}} \cdot\left\langle f_{E}^{\rho} \mid W\left(N_{E}\right), f_{E}\right\rangle_{p N_{E}} \\
&=\left\langle f_{E}^{\rho}\left|W\left(N_{E}\right)\right|\left(\begin{array}{cc}
p & 0 \\
0 & 1
\end{array}\right), f_{E}\right\rangle_{p N_{E}}-\beta_{p} p^{-1} \cdot\left\langle-w_{E} \cdot f_{E}, f_{E}\right\rangle_{p N_{E}} \\
&=\left\langle-w_{E} \cdot f_{E}\left|\left(\begin{array}{cc}
p & 0 \\
0 & 1
\end{array}\right)\right| \operatorname{Tr}_{\Gamma_{0}\left(N_{E}\right)}^{\Gamma_{0}\left(p N_{E}\right)}, f_{E}\right\rangle_{N_{E}}+w_{E} \beta_{p} p^{-1}\left[\Gamma_{0}\left(N_{E}\right): \Gamma_{0}\left(p N_{E}\right)\right] \cdot\left\langle f_{E}, f_{E}\right\rangle_{N_{E}} .
\end{aligned}
$$

Note from the trace map identity $f_{E}\left|\left(\begin{array}{cc}p & 0 \\ 0 & 1\end{array}\right)\right| \operatorname{Tr}_{\Gamma_{0}\left(N_{E}\right)}^{\Gamma_{0}\left(p N_{E}\right)}=f_{E} \mid T_{p}^{*}=\overline{a_{p}(E)} f_{E}$ together with the index formula $\left[\Gamma_{0}\left(N_{E}\right): \Gamma_{0}\left(p N_{E}\right)\right]=p+1$, the above becomes

$$
\left\langle f^{0}, f_{E}\right\rangle_{p N_{E}}=w_{E} \cdot\left(-a_{p}(E)+\beta_{p} \cdot \frac{p+1}{p}\right) \times\left\langle f_{E}, f_{E}\right\rangle_{N_{E}}=-w_{E} \cdot\left(\frac{\alpha_{p}^{2}-1}{\alpha_{p}}\right) \times\left\langle f_{E}, f_{E}\right\rangle_{N_{E}} .
$$

Lastly to prove that (iii) is true, one knows from Equation (2) that

$$
\left\langle f^{0}, f_{E}(p z)\right\rangle_{p N_{E}}=\left\langle f^{0}, \beta_{p}^{-1}\left(f_{E}(z)-f_{0}(z)\right)\right\rangle_{p N_{E}}=\beta_{p}^{-1} \times\left(\left\langle f^{0}, f_{E}\right\rangle_{p N_{E}}-\left\langle f^{0}, f_{0}\right\rangle_{p N_{E}}\right) .
$$

The first term $\left\langle f^{0}, f_{E}\right\rangle_{p N_{E}}$ is already determined from (ii) above. To compute the second term,

$$
\left\langle f^{0}, f_{0}\right\rangle_{p N_{E}}=\left(p \alpha_{p}\right)^{-1}\left(\alpha_{p}-\beta_{p}\right)\left(p \alpha_{p}-\beta_{p}\right) \cdot\left\langle f_{E}^{\rho} \mid W\left(N_{E}\right), f_{E}\right\rangle_{N_{E}}
$$

upon applying [18, §C.5, Lemma 1], and clearly one has $\left\langle f_{E}^{\rho} \mid W\left(N_{E}\right), f_{E}\right\rangle_{N_{E}}=-w_{E}\left\langle f_{E}, f_{E}\right\rangle_{N_{E}}$. Combining these strands together:

$$
\begin{aligned}
& \left\langle f^{0}, f_{E}(p z)\right\rangle_{p N_{E}}=\beta_{p}^{-1} \times\left(-w_{E} \cdot \frac{\alpha_{p}^{2}-1}{\alpha_{p}}+w_{E} \cdot \frac{\left(\alpha_{p}-\beta_{p}\right)\left(p \alpha_{p}-\beta_{p}\right)}{p \alpha_{p}}\right) \times\left\langle f_{E}, f_{E}\right\rangle_{N_{E}} \\
& =-w_{E} \cdot\left(\frac{\alpha_{p}^{2}-1}{p}-\frac{\left(\alpha_{p}-\beta_{p}\right)\left(p \alpha_{p}-\beta_{p}\right)}{p^{2}}\right) \times\left\langle f_{E}, f_{E}\right\rangle_{N_{E}}=-w_{E} \cdot\left(\frac{\alpha_{p}^{2}-1}{\alpha_{p}^{2}}\right) \times\left\langle f_{E}, f_{E}\right\rangle_{N_{E}}
\end{aligned}
$$

which completes the demonstration of (iii), and thereby the lemma.

\subsection{The $q$-expansion of the modular form $R_{m, e}$}

The key ingredient in calculating the first derivative of $\mathbf{L}_{p}^{\mathrm{imp}}\left(\operatorname{Sym}^{2} E, s\right)$ at $s=1$, is that the moments of the measure $\mathrm{d} \mu_{E}^{\mathrm{imp}}(-)$ can be written in terms of the $f^{0}$-isotypic projection of a holomorphic modular form. More precisely, let us recall from [7, Eqs (3.22)-(3.23)] that

$$
\mu_{E}^{\mathrm{imp}}\left(e+p^{m} \mathbb{Z}_{p}\right)=2 \alpha_{p}^{-2 m} \times \frac{\left\langle f^{0}, R_{m, e} \mid U_{p}^{2 m-1}\right\rangle_{p N_{E}}}{\left\langle f_{E}, f_{E}\right\rangle_{N_{E}}}
$$

where $R_{m, e} \in \mathcal{M}_{2}\left(\Gamma_{0}\left(p^{2 m} N_{E}\right)\right)$ is obtained by summing up products of certain theta-functions of weight $1 / 2$ with Eisenstein series of weight $3 / 2$ (the precise definitions will not be needed). Note also from [7, Lemma 3.10(ii)], the classical trace map identity

$$
h\left|U_{p}^{2 m-1}=h\right| W\left(p^{2 m} N_{E}\right)\left|\operatorname{Tr}_{\Gamma_{0}\left(p N_{E}\right)}^{\Gamma_{0}\left(p^{2 m} N_{E}\right)}\right| W\left(p N_{E}\right)
$$


implies that $R_{m, e} \mid U_{p}^{2 m-1}$ actually has level $p N_{E}$, so the inner product above is well-defined.

Remarks: (i) If $R_{m, e}=\sum_{n=0}^{\infty} r_{n}(m, e) q^{n}$ then clearly $R_{m, e} \mid U_{p}^{2 m-1}=\sum_{n=0}^{\infty} r_{n p^{2 m-1}}(m, e) q^{n}$; furthermore, $r_{0}(m, e)=0$ since the theta-functions of weight $1 / 2$ vanish at the cusp $\infty$.

(ii) Applying [7, Theorem 3.11] each coefficient $r_{n}(m, e) \in \mathbb{Q}$, in fact $r_{n}(m, e) \in \mathbb{Z}_{p}$ if $p^{2 m-1} \mid n$; it therefore follows that $R_{m, e} \mid U_{p}^{2 m-1} \in q \cdot \mathbb{Z}_{(p)}[[q]]$.

(iii) Assuming $p^{2 m-1}$ divides $n$, from [7, p133] the $q^{n}$-coefficient of $R_{m, e}$ is given by

$$
r_{n}(m, e)=\frac{-2}{\phi\left(p^{m}\right)} \times \sum_{\chi \in \Delta_{m}} \sum_{\left(n_{1}, n_{2}\right) \in \mathcal{W}_{n}} \sum_{(a, b) \in \mathcal{V}_{n_{2}}} \mu(a) b \cdot \varepsilon_{n_{2}}(a) \chi\left(b^{2} a\right) \chi^{-1}\left(n_{1} e\right) \cdot L_{N_{E}}\left(\chi \varepsilon_{n_{2}}, 0\right) .
$$

Here we have employed the notation:

- $\Delta_{m}$ denotes the set of non-trivial Dirichlet characters of conductor dividing $p^{m}$;

- $\mathcal{W}_{n}$ is the set of pairs $\left(n_{1}, n_{2}\right) \in \mathbb{N} \times \mathbb{N}$ coprime to $p$, and satisfying $n_{1}^{2} \times \frac{N_{E}}{4}+n_{2}=n$;

- $\mathcal{V}_{n_{2}}$ consists of pairs $(a, b) \in \mathbb{N} \times \mathbb{N}$ that are coprime to $p N_{E}$, such that $(a b)^{2}$ divides $n_{2}$;

- $\varepsilon_{n_{2}}$ refers to the character of the imaginary quadratic field $\mathbb{Q}\left(\sqrt{-n_{2} N_{E}}\right)$.

As usual, $L_{N_{E}}\left(\chi \varepsilon_{n_{2}}, s\right)$ indicates the $\chi \varepsilon_{n_{2}}$-twisted zeta-function with its Euler factors at the primes dividing $N_{E}$ removed.

Definition 2.3. (a) For an integer $t \geq 1$ and $y \in \mathbb{Z}$ with $p \nmid y$, one defines

$$
\vartheta_{t}(y)=\left\{\begin{array}{cl}
(p-1)^{2} / p^{2} & \text { if } t \geq 2 \text { and } y \equiv 1\left(\bmod p^{t}\right) \\
-(p-1) / p^{2} & \text { if } t \geq 2, y \neq \equiv 1\left(\bmod p^{t}\right) \text { but } y \equiv 1\left(\bmod p^{t-1}\right) \\
0 & \text { if } t \geq 2 \text { and } y \not \equiv 1\left(\bmod p^{t-1}\right) \\
(p-2) / p & \text { if } t=1 \text { and } y \equiv 1(\bmod p) \\
-1 / p & \text { if } t=1 \text { and } y \neq \equiv 1(\bmod p) .
\end{array}\right.
$$

(b) For any $m \in \mathbb{N}$ and integers $x, n_{2}$ both coprime to $p$, we set

$$
M_{m}^{\left(n_{2}\right)}(x):=\sum_{t=1}^{m} \sum_{\substack{j=1, p \nmid j}}^{p^{t}} p^{t} \cdot \vartheta_{t}(x j) \times \frac{-1}{\mathfrak{f}_{\varepsilon_{n_{2}}}} \cdot \sum_{i=0}^{\mathfrak{f}_{\varepsilon_{n_{2}}}-1} \varepsilon_{n_{2}}(i) \cdot\left((i-j) p^{-t}\right)^{\sharp}
$$

where $\left((i-j) p^{-t}\right)^{\sharp} \in\left\{0, \ldots, \mathfrak{f}_{\varepsilon_{n_{2}}}-1\right\}$ is the unique integer congruent to $(i-j) p^{-t} \bmod \mathfrak{f}_{\varepsilon_{n_{2}}}$.

The following yields an alternate expression for $r_{n}(m, e)$, designed for use in our programs.

Proposition 2.4. If $p^{2 m-1}$ divides $n$, then the $q^{n}$-coefficient of $R_{m, e}$ is given by

$$
r_{n}(m, e)=-2 \cdot \sum_{\left(n_{1}, n_{2}\right) \in \mathcal{W}_{n}} \sum_{(a, b) \in \mathcal{V}_{n_{2}}} \sum_{d \mid N_{E}} \mu(a d) b \varepsilon_{n_{2}}(a d) \times \phi\left(p^{m}\right)^{-1} \cdot M_{m}^{\left(n_{2}\right)}\left(a b^{2} d\left(n_{1} e\right)^{*}\right)
$$

where $\left(n_{1} e\right)^{*} \in\left\{1, \ldots, p^{m}-1\right\}$ denotes the multiplicative inverse of $n_{1}$ e modulo $p^{m}$.

Before we give the demonstration, we make a couple of observations.

Firstly, the main expense in computing $r_{n}(m, e)$ is in tabulating the values of $\varepsilon_{n_{2}}$ necessary to compute $M_{m}^{\left(n_{2}\right)}(-)$. The length of time required to compute $r_{n}(m, e)$ is roughly proportional to the sum $\sum_{\left(n_{1}, n_{2}\right) \in \mathcal{W}_{n}} \mathfrak{f}_{\varepsilon_{n_{2}}}$, which has order $O\left(p^{3 m}\right)$ as a function of $m$. 
Secondly, the quantity $\phi\left(p^{m}\right)^{-1} \cdot M_{m}^{\left(n_{2}\right)}\left(a b^{2} d\left(n_{1} e\right)^{*}\right)$ occurring above is actually $p$-integral. The reason is that $M_{m}^{\left(n_{2}\right)}(-)$ coincides with ' $M_{m}(-)$ ' defined in [7, Eq (3.30)], and then by Lemma 3.12 of $o p$. cit., the latter is congruent to zero modulo $p^{m-1}$. However, once one has programmed in the function $\vartheta_{t}$, our version $M_{m}^{\left(n_{2}\right)}(-)$ is the quicker to calculate numerically.

Proof. If one recalls the standard identity $L_{N_{E}}\left(\chi \varepsilon_{n_{2}}, s\right)=\sum_{d \mid N_{E}} \mu(d) \chi(d) \varepsilon_{n_{2}}(d) d^{-s} \cdot L\left(\chi \varepsilon_{n_{2}}, s\right)$, then Equation (5) can be rewritten as

$$
r_{n}(m, e)=\frac{-2}{\phi\left(p^{m}\right)} \times \sum_{\left(n_{1}, n_{2}\right) \in \mathcal{W}_{n}} \sum_{(a, b) \in \mathcal{V}_{n_{2}}} \sum_{d \mid N_{E}} \mu(a d) b \cdot \varepsilon_{n_{2}}(a d) \cdot \sum_{\chi \in \Delta_{m}} \chi\left(\frac{a b^{2} d}{n_{1} e}\right) L\left(\chi \varepsilon_{n_{2}}, 0\right) .
$$

Therefore, it is enough to show that $\sum_{\chi \in \Delta_{m}} \chi(x) L\left(\chi \varepsilon_{n_{2}}, 0\right)$ is equal to the quantity $M_{m}^{\left(n_{2}\right)}(x)$. Now as each $L\left(\chi \varepsilon_{n_{2}}, 0\right)=-B_{1, \chi \varepsilon_{n_{2}}}$ with $B_{1, \chi \varepsilon_{n_{2}}}$ denoting a $\chi \varepsilon_{n_{2}}$-twisted Bernoulli number,

$$
L\left(\chi \varepsilon_{n_{2}}, 0\right)=\frac{-1}{\mathfrak{f}_{\chi} \mathfrak{f}_{\varepsilon_{n_{2}}}} \cdot \sum_{a=1}^{\mathfrak{f}_{\chi} \mathfrak{f}_{\varepsilon_{n_{2}}}} \chi \varepsilon_{n_{2}}(a) \cdot a=\frac{-1}{\mathfrak{f}_{\varepsilon_{n_{2}}}} \cdot p^{-t} \times \sum_{i=1}^{\mathfrak{f}_{\varepsilon_{n_{2}}}} \sum_{j=1}^{p^{t}} \chi\left(a_{i, j}\right) \varepsilon_{n_{2}}\left(a_{i, j}\right) \cdot a_{i, j}
$$

where $\mathfrak{f}_{\chi}=p^{t}>1$ say, and the integers $a_{i, j}:=(i-1) p^{t}+j$. Moreover $\chi\left(a_{i, j}\right)=\chi(j)$, so decomposing $\Delta_{m}$ into a disjoint union of $\Delta_{t}-\Delta_{t-1}$ 's yields

$$
\begin{aligned}
\sum_{\chi \in \Delta_{m}} \chi(x) L\left(\chi \varepsilon_{n_{2}}, 0\right) & =\sum_{t=1}^{m} \sum_{\chi \in \Delta_{t}-\Delta_{t-1}} \chi(x) L\left(\chi \varepsilon_{n_{2}}, 0\right) \\
& =\sum_{t=1}^{m} \sum_{\substack{j=1, p \nmid j}}^{p^{t}}\left(p^{-t} \cdot \sum_{\chi \in \Delta_{t}-\Delta_{t-1}} \chi(x j)\right) \times \frac{-1}{\mathfrak{f}_{\varepsilon_{n_{2}}}} \cdot \sum_{i=1}^{\mathfrak{f}_{n_{2}}} \varepsilon_{n_{2}}\left(a_{i, j}\right) \cdot a_{i, j} .
\end{aligned}
$$

The lemma will now follow, provided one can verify that:

(i) $p^{-t} \cdot \sum_{\chi \in \Delta_{t}-\Delta_{t-1}} \chi(x j)$ equals $\vartheta_{t}(x j)$;

(ii) $\sum_{i=1}^{\mathfrak{f}_{\varepsilon_{2}}} \varepsilon_{n_{2}}\left(a_{i, j}\right) \cdot a_{i, j}$ coincides with $p^{t} \cdot \sum_{i=0}^{\mathfrak{f}_{\varepsilon_{n_{2}}}-1} \varepsilon_{n_{2}}(i) \cdot\left((i-j) p^{-t}\right)^{\sharp}$.

To establish statement (i), if $\bar{\Delta}_{t}=\Delta_{t} \cup\{\mathbf{1}\}$ for $t>0$ with $\bar{\Delta}_{0}=\{\mathbf{1}\}$ then

$$
\begin{aligned}
p^{-t} \cdot \sum_{\chi \in \Delta_{t}-\Delta_{t-1}} \chi(x j) & =p^{-t} \cdot\left(\sum_{\chi \in \bar{\Delta}_{t}} \chi(x j)-\sum_{\chi \in \bar{\Delta}_{t-1}} \chi(x j)\right) \\
& =p^{-t} \cdot\left(\phi\left(p^{t}\right) \times \operatorname{char}_{1 \bmod } p^{t}(x j)-\phi\left(p^{t-1}\right) \times \operatorname{char}_{1 \bmod p^{t-1}}(x j)\right)
\end{aligned}
$$

where $\operatorname{char}_{1 \bmod p^{t}}(y)$ returns 1 if $p^{t}$ divides $y-1$, and returns 0 otherwise. It is then routine to check that the above formula agrees with $\vartheta_{t}(x j)$ from Definition 2.3.

To prove that (ii) is true, we first observe that

$$
\sum_{i=1}^{\mathfrak{f}_{\varepsilon_{n_{2}}}} \varepsilon_{n_{2}}\left(a_{i, j}\right) \cdot a_{i, j}=p^{t} \sum_{i=0}^{\mathfrak{f}_{\varepsilon_{n_{2}}}-1} \varepsilon_{n_{2}}\left(i p^{t}+j\right) \cdot i+j \sum_{i=0}^{\mathfrak{f}_{\varepsilon_{n_{2}}}-1} \varepsilon_{n_{2}}\left(i p^{t}+j\right)
$$

and the right-most summation is identically zero. Furthermore

$$
p^{t} \cdot \sum_{i=0}^{\mathfrak{f}_{\varepsilon_{2}}-1} \varepsilon_{n_{2}}\left(i p^{t}+j\right) \cdot i=p^{t} \cdot \sum_{i=0}^{\mathfrak{f}_{\varepsilon_{n_{2}}}-1} \varepsilon_{n_{2}}(i) \cdot\left((i-j) p^{-t}\right)^{\sharp}
$$

so statement (ii) is also verified. 


\subsection{Expressing $R_{m, e} \mid U_{p}^{2 m-1}$ in terms of a rational basis}

The next stage is to write $R_{m, e} \mid U_{p}^{2 m-1}$ in terms of an explicit rational basis of $\mathcal{M}_{2}\left(\Gamma_{0}\left(p N_{E}\right)\right)$. One first uses the decomposition

$$
\mathcal{M}_{2}\left(\Gamma_{0}\left(p N_{E}\right)\right)=\mathcal{S}_{2}\left(\Gamma_{0}\left(p N_{E}\right)\right) \oplus \operatorname{Eis}_{2}\left(\Gamma_{0}\left(p N_{E}\right)\right)
$$

where the second summand denotes the space of generalised Eisenstein series of weight two, level $p N_{E}$ and trivial nebentypus.

Definition 2.5. For two Dirichlet characters $\chi$ and $\psi$, we define the $q$-expansion

$$
\mathcal{E}_{2}(\chi, \psi)(q)=c_{0}(\chi, \psi) q^{0}+\sum_{m=1}^{\infty}\left(\sum_{d \mid m} \psi(d) \chi(m / d) \cdot d\right) q^{m} \in \overline{\mathbb{Q}}[[q]]
$$

where the constant term $c_{0}(\chi, \psi)= \begin{cases}0 & \text { if } \mathfrak{f}_{\chi}>1 \\ -B_{2, \psi} / 4 & \text { if } \mathfrak{f}_{\chi}=1 .\end{cases}$

Let $\mathfrak{X}_{p N_{E}}$ be the group of Dirichlet characters $\chi:\left(\mathbb{Z} / p N_{E} \mathbb{Z}\right)^{\times} \rightarrow \mathbb{C}^{\times}$. One can then form sets

$$
\begin{aligned}
& \Sigma_{1}:=\left\{\mathcal{E}_{2}(\mathbf{1}, \mathbf{1})(q)-c \cdot \mathcal{E}_{2}(\mathbf{1}, \mathbf{1})\left(q^{c}\right) \text { where } c \in \mathbb{N}, c \neq 1 \text { and } c \mid p N_{E}\right\}, \quad \text { and } \\
& \Sigma_{2}:=\left\{\mathcal{E}_{2}\left(\chi, \chi^{-1}\right)\left(q^{c}\right) \text { where } c \in \mathbb{N}, \chi \in \mathfrak{X}_{p N_{E}}-\{\mathbf{1}\} \text { and } c \mathfrak{f}_{\chi}^{2} \mid p N_{E}\right\} .
\end{aligned}
$$

Remember throughout we have assumed the conductor $N_{E}$ is divisible by 4 , and that $p \nmid N_{E}$. The following is a special case of a very well known result - the full details can be found in Miyake's book [28, Thm 4.7.1 and $\S 7.2]$.

Proposition 2.6. (i) $\operatorname{dim}_{\mathbb{C}}\left(\operatorname{Eis}_{2}\left(\Gamma_{0}\left(p N_{E}\right)\right)\right)=2 \cdot \sum_{d \mid N_{E}} \phi\left(\operatorname{gcd}\left(d, N_{E} / d\right)\right)-1$;

(ii) The union $\Sigma_{1} \cup \Sigma_{2}$ consists of Hecke eigenforms, and yields a $\mathbb{C}$-basis for $\operatorname{Eis}_{2}\left(\Gamma_{0}\left(p N_{E}\right)\right)$.

Turning our attention to the space of cusp forms,

$$
\mathcal{S}_{2}\left(\Gamma_{0}\left(p N_{E}\right)\right) \cong \bigoplus_{M \mid p N_{E}} \bigoplus_{c \mid p N_{E} / M} \mathcal{S}_{2}^{\text {new }}\left(\Gamma_{0}(M)\right) \mid V_{c}
$$

and one can express an arbitrary cusp form as a linear combination of Hecke eigenforms.

Proposition 2.7. Since 4 divides $N_{E}$, the size of the space of cusp forms is given by

$$
\operatorname{dim}_{\mathbb{C}}\left(\mathcal{S}_{2}\left(\Gamma_{0}\left(p N_{E}\right)\right)\right)=1+\left(\frac{p+1}{12}\right) \cdot \prod_{l \mid N_{E}}(l+1) \cdot l^{\operatorname{ord}_{l}\left(N_{E}\right)-1}-\sum_{d \mid N_{E}} \phi\left(\operatorname{gcd}\left(d, N_{E} / d\right)\right) .
$$

Proof. This follows immediately from a classical formula of Shimura (e.g. see [34, Prop 6.1]), upon noting that $4 \mid p N_{E}$ and $p$ exactly divides the level $p N_{E}$.

Write $\mathbf{d}_{\mathcal{S}}$ for the dimension of $\mathcal{S}_{2}\left(\Gamma_{0}\left(p N_{E}\right)\right)$, and let $\mathbf{d}_{\text {Eis }}$ be the dimension of $\operatorname{Eis}_{2}\left(\Gamma_{0}\left(p N_{E}\right)\right)$. Then there exist coefficients $\delta_{\bullet}(m, e) \in \mathbb{Q}$ such that

$$
R_{m, e} \mid U_{p}^{2 m-1}=\delta_{1}(m, e) \cdot f_{E}(z)+\delta_{2}(m, e) \cdot f_{E}(p z)+\sum_{i=3}^{\mathbf{d}_{\mathcal{S}}} \delta_{i}(m, e) \cdot g_{i}+\sum_{j=1}^{\mathbf{d}_{\mathrm{Eis}}} \delta_{j+\mathbf{d}_{\mathcal{S}}}(m, e) \cdot h_{j}
$$


where $\left\{f_{E}(z), f_{E}(p z), g_{3}(z), g_{4}(z), \ldots, g_{\mathbf{d}_{\mathcal{S}}}(z)\right\}$ is a basis of cuspidal eigenforms at level $p N_{E}$, and $\left\{h_{1}, \ldots, h_{\mathbf{d}_{\mathrm{Eis}}}\right\}$ denotes an arbitrary $\mathbb{Q}$-basis for the Eisenstein component.

Remarks: (a) We have adopted the labelling convention that $g_{1}(z)=f_{E}(z)$ and $g_{2}(z)=f_{E}(p z)$. (b) To find the basis elements $g_{1}, \ldots g_{\mathbf{d}_{\mathcal{S}}}$ generating the space of cusp forms, at every $M \mid p N_{E}$ we compute a basis of $q$-expansions for $\mathcal{S}_{2}^{\text {new }}\left(\Gamma_{0}(M)\right)$, by using the SAGE command

\section{CuspForms (Gamma0 (N) , k) .new_subspace ().q_expansion_basis (prec)}

with level $N=M$ and for weight $k=2$. We then compute $g\left(q^{c}\right)$ for each $g \in \mathcal{S}_{2}^{\text {new }}\left(\Gamma_{0}(M)\right)$ and $c$ dividing $p N_{E} / M$ (note the precision of the $q$-expansions is determined by the value of prec).

(c) To find the elements $h_{1}, \ldots, h_{\mathbf{d}_{\text {Eis }}}$ one can code up an implementation of Proposition 2.6(ii), or instead produce a basis of $q$-expansions (in echelon form) via the SAGE command

EisensteinForms (Gamma0 $(\mathrm{N}), \mathrm{k})$.q_expansion_basis (prec)

with level $N=p N_{E}$ and for weight $k=2$, once again.

We are then left with the task of determining the $\delta_{\bullet}(m, e)$ 's, especially $\delta_{1}(m, e)$ and $\delta_{2}(m, e)$. To

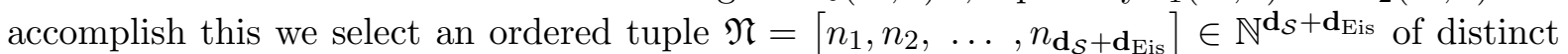
positive integers, then consider the $\left(\mathbf{d}_{\mathcal{S}}+\mathbf{d}_{\text {Eis }}\right) \times\left(\mathbf{d}_{\mathcal{S}}+\mathbf{d}_{\text {Eis }}\right)$-linear system of equations

$$
r_{n p^{2 m-1}}(m, e)=\sum_{i=1}^{\mathbf{d}_{\mathcal{S}}} a_{n}\left(g_{i}\right) \cdot \delta_{i}(m, e)+\sum_{j=1}^{\mathbf{d}_{\text {Eis }}} a_{n}\left(h_{j}\right) \cdot \delta_{j+\mathbf{d}_{\mathcal{S}}}(m, e) \quad \text { for each } n \in \mathfrak{N}
$$

arising from Equation (6). The corresponding $q$-coefficient matrix is given by

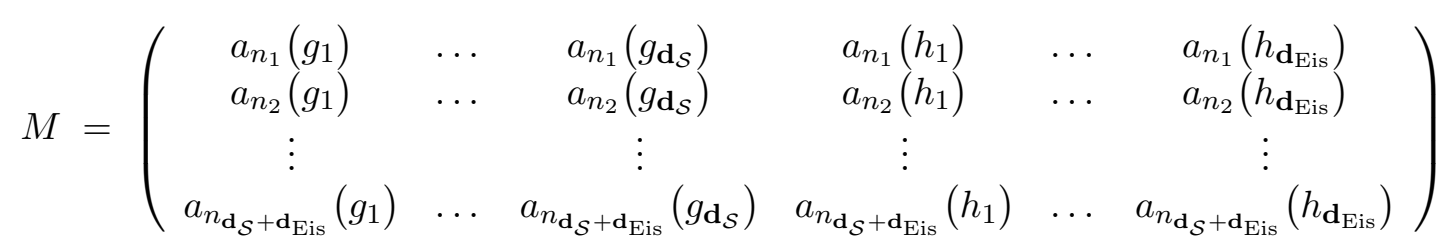

so we can write the system as $\underline{r(m, e)^{T}}=M \times \underline{\delta(m, e)^{T}}$, where $\underline{r(m, e)}=\left(r_{n p^{2 m-1}}(m, e)\right)_{n \in \mathfrak{N}}$ and $\underline{\delta(m, e)}=\left(\delta_{i}(m, e)\right)_{i=1, \ldots, \# \mathfrak{N}}$

Hypothesis $(\operatorname{det} M \neq 0)$. The matrix $M=M(\mathfrak{N})$ is invertible for the choice of tuple $\mathfrak{N}$.

Clearly one can always find an $\mathfrak{N}$ for which the above holds, otherwise $\left\{g_{1}, \ldots, g_{\mathbf{d}_{\mathcal{S}}}, h_{1}, \ldots, h_{\mathbf{d}_{\text {Eis }}}\right\}$ would not be a basis for $\mathcal{M}_{2}\left(\Gamma_{0}\left(p N_{E}\right)\right)$. In practice, we choose a tuple $\mathfrak{N}$ that will minimise $\sum_{n \in \mathfrak{N}} \sum_{\left(n_{1}, n_{2}\right) \in \mathcal{W}_{n}} \mathfrak{f}_{\varepsilon_{n_{2}}}$, and hence the time needed to compute the vector $\underline{r(m, e)}$.

Corollary 2.8. If Hypothesis ( $\operatorname{det} M \neq 0$ ) is satisfied for a tuple $\mathfrak{N}$, and $W=\left(w_{i, j}\right)_{1 \leq i, j \leq \# \mathfrak{N}}$ denotes the inverse matrix to $M=M(m, e, \mathfrak{N})$, then $\underline{\delta(m, e)^{T}}=W \times \underline{r(m, e)^{T}}$; in particular

$$
\delta_{1}(m, e)=\sum_{j=1}^{\# \mathfrak{N}} w_{1, j} \cdot \underline{r(m, e)} j \quad \text { and } \quad \delta_{2}(m, e)=\sum_{j=1}^{\# \mathfrak{N}} w_{2, j} \cdot \underline{r(m, e)} j .
$$

Therefore, to obtain these first two components of $\delta(m, e)$, we must:

- compute the dimensions $\mathbf{d}_{\mathcal{S}}$ and $\mathbf{d}_{\text {Eis }}$ by using Propositions 2.7 and 2.6(i), respectively;

- calculate $g_{1}, \ldots g_{\mathbf{d}_{\mathcal{S}}}$ and $h_{1}, \ldots, h_{\mathbf{d}_{\mathrm{Eis}}}$ using the SAGE commands in (b) and (c) above; 
- find an optimal choice of $\mathfrak{N} \in \mathbb{N}^{\mathbf{d}_{\mathcal{S}}+\mathbf{d}_{\text {Eis }}}$ such that Hypothesis ( $\operatorname{det} M \neq 0$ ) holds;

- produce the vector of $q$-coefficients $\underline{r(m, e)}=\left(r_{n p^{2 m-1}}(m, e)\right)_{n \in \mathfrak{N}}$ from Propositon 2.4;

- evaluate the first two basis coefficients, i.e. $\delta_{1}(m, e)$ and $\delta_{2}(m, e)$, using Corollary 2.8 .

The slowest part of the algorithm is the penultimate line, and as we need $\# \mathfrak{N}=\mathbf{d}_{\mathcal{S}}+\mathbf{d}_{\text {Eis }}$ of these $r_{n p^{2 m-1}}(m, e)$ 's, the time required for this step has order $O\left(\left(\mathbf{d}_{\mathcal{S}}+\mathbf{d}_{\text {Eis }}\right) \times p^{3 m}\right)$.

\subsection{An explicit formula for $\mathcal{L}_{p}^{\text {an }}\left(\operatorname{Sym}^{2} E\right)$ modulo $p^{m}$, when $D(E, 1) \neq 0$}

We shall begin by expressing the moments of the measure $\mathrm{d} \mu_{E}^{\mathrm{imp}}$ in terms of the vector $\delta(m, e)$. For each $m \geq 1$, define an integer $\nu_{m, p}=\nu_{m, p}\left(\mathcal{F}^{\text {imp }}\right)$ by the rule

$$
\nu_{m, p}\left(\mathcal{F}^{\mathrm{imp}}\right):=\min \left\{\operatorname{ord}_{p}\left(\delta_{1}(m, e)\right), \operatorname{ord}_{p}\left(\delta_{2}(m, e)\right) \text { where } e \in\left(\mathbb{Z} / p^{m} \mathbb{Z}\right)^{\times}\right\} .
$$

Therefore to compute $\nu_{m, p}$ we must calculate the $2(p-1) p^{m-1}$ coefficients $\delta_{\bullet}(m, e), \bullet=1,2$.

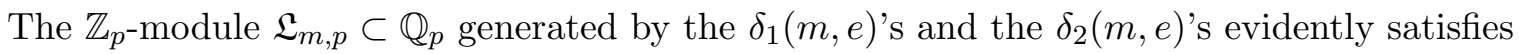

$$
\mathfrak{L}_{m, p}:=\mathbb{Z}_{p} \cdot\left\langle\delta_{1}(m, e), \delta_{2}(m, e) \mid e \in\left(\mathbb{Z} / p^{m} \mathbb{Z}\right)^{\times}\right\rangle=p^{\nu_{m, p}} \cdot \mathbb{Z}_{p}
$$

In particular, if the values $\delta_{\bullet}(m, e)$ 's are $p$-integral then $\mathfrak{L}_{m, p} \subset \mathbb{Z}_{p}$, whence $\nu_{m, p}\left(\mathcal{F}^{\text {imp }}\right) \geq 0$.

Lemma 2.9. For each integer $m \geq 1$ and congruence class $e \in\left(\mathbb{Z} / p^{m} \mathbb{Z}\right)^{\times}$,

$$
\mu_{E}^{\mathrm{imp}}\left(e+p^{m} \mathbb{Z}_{p}\right)=\frac{-2 w_{E}}{\alpha_{p}^{2 m}} \cdot\left(1-\alpha_{p}^{-2}\right) \times\left(\alpha_{p} \cdot \delta_{1}(m, e)+\delta_{2}(m, e)\right)
$$

and these moments lie inside $p^{\nu_{m, p}}\left(1-\alpha_{p}^{-2}\right) \cdot \mathbb{Z}_{p}$.

Proof. Considering Equations (4) and (6) in turn, one deduces that

$$
\begin{aligned}
\mu_{E}^{\mathrm{imp}}\left(e+p^{m} \mathbb{Z}_{p}\right) & =2 \alpha_{p}^{-2 m} \times \frac{\left\langle f^{0}, R_{m, e} \mid U_{p}^{2 m-1}\right\rangle_{p N_{E}}}{\left\langle f_{E}, f_{E}\right\rangle_{N_{E}}} \\
& =2 \alpha_{p}^{-2 m} \times \frac{\left\langle f^{0}, \delta_{1}(m, e) f_{E}(z)+\delta_{2}(m, e) f_{E}(p z)+\widetilde{R}(z)\right\rangle_{p N_{E}}}{\left\langle f_{E}, f_{E}\right\rangle_{N_{E}}}
\end{aligned}
$$

where $\widetilde{R}(z) \in \mathcal{M}_{2}\left(\Gamma_{0}\left(p N_{E}\right)\right)$ intersects trivially with the isotypic subspace $\left(\mathbb{C} \cdot f_{E} \oplus \mathbb{C} \cdot f_{E}(p z)\right)$. If we make full use of Lemma 2.2, the three Petersson inner product identities imply

$\mu_{E}^{\operatorname{imp}}\left(e+p^{m} \mathbb{Z}_{p}\right)=2 \alpha_{p}^{-2 m} \times\left(\delta_{1}(m, e) \cdot\left(-w_{E} \cdot \frac{\alpha_{p}^{2}-1}{\alpha_{p}}\right)+\delta_{2}(m, e) \cdot\left(-w_{E} \cdot \frac{\alpha_{p}^{2}-1}{\alpha_{p}^{2}}\right)+0\right)$

which is equivalent to the stated formula.

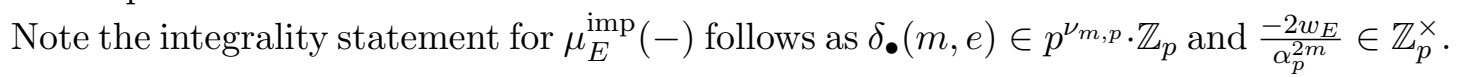

An important corollary of this result is that the power series $\mathcal{F}^{\text {imp }}(X)$ belongs to $p^{\nu_{m, p}} \cdot \mathbb{Z}_{p} \llbracket X \rrbracket$, hence the imprimitive $p$-adic $L$-function is $p$-integral if $\left|\delta_{1}(m, e)\right|_{p},\left|\delta_{2}(m, e)\right|_{p} \leq 1$ for all $e$. Furthermore, if $S_{\text {ord }}$ denotes the set of primes where $E$ has good ordinary reduction over $\mathbb{Q}_{p}$, 
and $S_{\text {denom }}$ consists of those primes dividing $6 \times \prod_{l \in S_{1}} H_{l}\left(l^{-1}\right) \times \frac{(2 \pi i)^{-1} \Omega_{E}^{+} \Omega_{E}^{-}}{\pi\left\langle f_{E}, f_{E}\right\rangle_{N_{E}}} \quad$ (cf. Lemma 2.1), an easy exercise verifies that

$$
\mathcal{F}(X) \in p^{\nu_{m, p}} \cdot \mathbb{Z}_{p} \llbracket X \rrbracket \quad \text { at every prime } p \in S_{\text {ord }}-S_{\text {denom }} .
$$

Consequently, the primitive $p$-adic $L$-function $\mathbf{L}_{p}\left(\operatorname{Sym}^{2} E, s\right)$ is a $p$-integral Iwasawa function at good ordinary primes $p \notin S_{\text {denom }}$ for which $\sup \left\{\nu_{m, p}\left(\mathcal{F}^{\text {imp }}\right) \mid m \in \mathbb{N}\right\} \geq 0$.

For each $m$, the quantities $\nu_{m, p}$ give a lower bound on the $\mu$-invariant of $\mathcal{F}(X)$ when $p \notin S_{\text {denom }}$. In all of our numerical calculations, we found that the exponent $\nu_{m, p}\left(\mathcal{F}^{\text {imp }}\right)$ stabilized as a function of $m \geq 3$, and was only once smaller than -2 in value. In fact, this was the single instance where $\mathbf{L}_{p}^{\prime}\left(\operatorname{Sym}^{2} E, 1\right) \notin \mathbb{Z}_{p}$, occurring at the prime $p=3$ for the curve $E=268 \mathrm{a} 1$.

Theorem 2.10. Provided that $D(E, 1) \neq 0$, if one defines $\xi_{\mathrm{Sym}^{2} E}:=\frac{D(E, 1)}{\pi\left\langle f_{E}, f_{E}\right\rangle_{N_{E}}}$ and sets $\epsilon_{p}=\operatorname{ord}_{p}\left(\left(1-\alpha_{p}^{-2}\right) \cdot \xi_{\mathrm{Sym}^{2} E}\right)$, then the $\mathcal{L}$-invariant will satisfy the congruences

$\mathcal{L}_{p}^{\mathrm{an}}\left(\operatorname{Sym}^{2} E\right) \equiv \frac{-2 w_{E} \cdot \xi_{\mathrm{Sym}^{2} E}^{-1}}{\alpha_{p}^{2 m}\left(1-p \alpha_{p}^{-2}\right)} \times \sum_{e \in\left(\mathbb{Z} / p^{m} \mathbb{Z}\right)^{\times}} \log _{p}<e>_{p} \cdot\left(\alpha_{p} \cdot \delta_{1}(m, e)+\delta_{2}(m, e)\right) \bmod p^{m+\nu_{m, p}-\epsilon_{p}}$ for every integer $m \geq 1$.

Proof. From Lemma 2.9, our formulae for the moments of the measure $\mathrm{d} \mu_{E}$ imply that

$$
\begin{aligned}
&\left.\frac{\mathrm{d}}{\mathrm{d} s} \mathbf{L}_{p}^{\mathrm{imp}}\left(\operatorname{Sym}^{2} E, s\right)\right|_{s=1}=\int_{x \in \mathbb{Z}_{p}^{\times}} \log _{p}<x>_{p} \cdot \mathrm{d} \mu_{E}^{\mathrm{imp}}(x) \\
& \equiv \sum_{e \in\left(\mathbb{Z} / p^{m} \mathbb{Z}\right)^{\times}} \log _{p}<e>_{p} \cdot \mu_{E}^{\mathrm{imp}}\left(e+p^{m} \mathbb{Z}_{p}\right) \quad \bmod p^{m+\nu_{m, p}} \\
& \equiv \frac{-2 w_{E}}{\alpha_{p}^{2 m}} \cdot\left(1-\alpha_{p}^{-2}\right) \times \sum_{e \in\left(\mathbb{Z} / p^{m} \mathbb{Z}\right)^{\times}} \log _{p}<e>_{p} \cdot\left(\alpha_{p} \cdot \delta_{1}(m, e)+\delta_{2}(m, e)\right) \bmod p^{m+\nu_{m, p}} .
\end{aligned}
$$

Now using Equation $(1)$ which is valid as $D(E, 1) \neq 0$, the $\mathcal{L}$-invariant can be expressed as

$$
\mathcal{L}_{p}^{\mathrm{an}}\left(\operatorname{Sym}^{2} E\right)=\left(\left(1-\alpha_{p}^{-2}\right)\left(1-p \alpha_{p}^{-2}\right) \xi_{\mathrm{Sym}^{2} E}\right)^{-1} \times\left.\frac{\mathrm{d}}{\mathrm{d} s} \mathbf{L}_{p}^{\mathrm{imp}}\left(\operatorname{Sym}^{2} E, s\right)\right|_{s=1}
$$

and since $\left(1-\alpha_{p}^{-2}\right)\left(1-p \alpha_{p}^{-2}\right) \xi_{\mathrm{Sym}^{2} E} \cdot \mathbb{Z}_{p}=p^{\epsilon_{p}} \cdot \mathbb{Z}_{p}$, the result follows directly.

\subsection{Attempts at evaluating the moments $\int x^{j} \cdot \mathrm{d} \mu_{E}^{\mathrm{imp}}$ for $j \neq 0$ ?}

Theoretically at least, there should be a more efficient way to compute the derivative of the imprimitive $p$-adic $L$-function at $s=1$, which we now outline. Keeping our previous notation,

$$
\begin{aligned}
\frac{\mathrm{d} \mathbf{L}_{p}^{\mathrm{imp}}\left(\mathrm{Sym}^{2} E, s\right)}{\mathrm{d} s} & =\int_{\mathbb{Z}_{p}^{\times}}\left\langle x>_{p}^{s-1} \log _{p}<x>_{p} \cdot \mathrm{d} \mu_{E}^{\mathrm{imp}}(x)\right. \\
& =\sum_{e=1}^{p-1} \sum_{j=0}^{\infty} \mathcal{A}_{e, j}(s) \cdot \int_{e+p \mathbb{Z}_{p}} x^{j} \cdot \mathrm{d} \mu_{E}^{\mathrm{imp}}(x)
\end{aligned}
$$

where $\sum_{j=0}^{\infty} \mathcal{A}_{e, j}(s) x^{j}$ is the power series development for $\left\langle x>_{p}^{s-1} \log _{p}<x>_{p}\right.$ along $e+p \mathbb{Z}_{p}$.

Question. Is there an efficient algorithm to determine $\int_{e+p \mathbb{Z}_{p}} x^{j} \cdot \mathrm{d} \mu_{E}^{\mathrm{imp}}(x)$ when $j \neq 0$ ? 
If there is a positive answer, then one simply needs to evaluate $\sum_{j=0}^{\infty} \mathcal{A}_{e, j}(1) \cdot \int_{e+p \mathbb{Z}_{p}} x^{j} \cdot \mathrm{d} \mu_{E}^{\text {imp }}$ to some prescribed $p$-adic precision, and next sum the values over the range $e=1, \ldots, p-1$. In theory this should yield a far quicker and more accurate method than using Riemann sums, but in practice there are a number of difficulties that arise.

To better illustrate these difficulties, let us assume that $\mathcal{F}_{k}$ is a $p$-stabilised ordinary Hecke eigenform of weight $k \geq 2$ and level $N p$. The critical points for the $L$-function of the symmetric square of $\mathcal{F}_{k}$ are $\{1, \ldots, 2 k-2\}$ which, after $p$-adically interpolating $L^{\mathrm{imp}}\left(\mathrm{Sym}^{2} \mathcal{F}_{k}, s\right)$ at positive integer values, naturally subdivide into the disjoint subsets $\{1, \ldots, k-1\}$ and $\{k, \ldots, 2 k-2\}$. If $\mathrm{d} \mu_{\mathrm{Sym}^{2} \mathcal{F}_{k}(j)}^{\mathrm{imp},-}$ is the measure interpolating $\chi$-twists of $\operatorname{Sym}^{2} \mathcal{F}_{k}(j)$ at each $j \in\{1, \ldots, k-1\}$, then the analytic methods in $[9,31]$ imply for some non-zero constant $c_{k} \in \overline{\mathbb{Q}}^{\times}$:

$$
\int_{e+p^{m} \mathbb{Z}_{p}} x^{j-1} \cdot \mathrm{d} \mu_{\mathrm{Sym}^{2} \mathcal{F}_{k}(1)}^{\mathrm{imp},-}(x)=\mu_{\mathrm{Sym}^{2} \mathcal{F}_{k}(j)}^{\mathrm{imp},-}\left(e+p^{m} \mathbb{Z}_{p}\right)=c_{k} \times \frac{\left\langle\mathcal{F}_{k}^{0}, \operatorname{Hol}\left(\tilde{R}_{m, e}^{(k, j)}\right) \mid U_{p}^{2 m-1}\right\rangle_{p N}}{\left\langle\mathcal{F}_{k}, \mathcal{F}_{k}\right\rangle_{p N}}
$$

where $\tilde{R}_{m, e}^{(k, j)}$ are certain $\mathcal{C}^{\infty}$-modular forms exhibiting moderate growth at the cusps of $X_{1}\left(p^{2 m} N\right)$, and 'Hol' denotes the operator of holomorphic projection, in the terminology of [20].

Remarks: (i) If $j=k-1$ then the modular forms $\tilde{R}_{m, e}^{(k, k-1)}$ are already holomorphic, and there is no need to hit them with the operator 'Hol' (e.g. for weight $k=2$, one has $R_{m, e}=\tilde{R}_{m, e}^{(2,1)}$ ).

(ii) However if $j \in\{1, \ldots, k-1\}$ and $j \neq k-1$, then $\tilde{R}_{m, e}^{(k, j)}$ is not a holomorphic modular form.

(iii) More alarmingly, if $j \in \mathbb{Z}-\{1, \ldots, k-1\}$ then $\tilde{R}_{m, e}^{(k, j)}$ no longer has moderate growth at the cusps of $X_{1}\left(p^{2 m} N\right)$, so attempting to evaluate $\operatorname{Hol}\left(\tilde{R}_{m, e}^{(k, j)}\right)$ does not even make sense.

For each critical value $j \in\{1, \ldots, k-1\}$, the Fourier expansion of $\operatorname{Hol}\left(\tilde{R}_{m, e}^{(k, j)}\right) \mid U_{p}^{2 m-1}$ can be readily computed [9, pp.592-594], and is of the form

$$
\operatorname{Hol}\left(\tilde{R}_{m, e}^{(k, j)}\right) \mid U_{p}^{2 m-1}=\sum_{n=1}^{\infty}\left(\sum_{p^{2 m-1} n=N n_{1}^{2}+n_{2}} C_{n_{2}, m, n}^{(k, j)} \times \int_{x \in e+p^{m} \mathbb{Z}_{p}} x^{j-k+1} \cdot \mathrm{d} \mu^{-}\left(x, \varepsilon_{n_{2}}\right)\right) \cdot q^{n}
$$

where the scalars $C_{n_{2}, m, n}^{(k, j)} \in \overline{\mathbb{Q}}$, and $\mathrm{d} \mu^{-}\left(x, \varepsilon_{n_{2}}\right)$ is the twisted Kubota-Leopoldt pseudo-measure interpolating $\int_{\mathbb{Z}_{p}^{\times}} \chi x^{s} \cdot \mathrm{d} \mu^{-}\left(x, \varepsilon_{n_{2}}\right)=\zeta_{p}\left(s, \chi^{-1} \varepsilon_{n_{2}}\right)$ at finite order characters $\chi$, with $1-s \in \mathbb{N}$.

In order to evaluate $\int x^{j} \cdot \mathrm{d} \mu \mu_{\mathrm{Sym}^{2} \mathcal{F}_{k}(k-1)}^{\mathrm{imp},-}$, one could naively try to Tate twist the $q$-expansions in Equation (7) at integer values $j \notin[1, k-1]$, and then compute the $\mathcal{F}_{k}^{0}$-isotypic component. We attempted this for both the ranges $j>k-1$ and $j<1$ (which lie outside the region of $p$-adic interpolation), but found that the corresponding $q$-expansions could not possibly come from modular forms of level $N p$. Essentially these methods fail because the operator 'Hol' cannot be extended to real analytic forms that do not exhibit moderate growth.

A possible salvage is to allow the $p$-stabilised eigenform $\mathcal{F}_{k}$ to vary in an ordinary family. For example, one could pick another weight $k^{\prime}=k+t(p-1) p^{r}$ for some $t, r \in \mathbb{N}$, and a Hecke eigenform $\mathcal{F}_{k^{\prime}} \in \mathcal{S}_{k^{\prime}}\left(\Gamma_{1}(N p)\right)$ such that $\mathcal{F}_{k^{\prime}} \equiv \mathcal{F}_{k} \bmod p^{r}$. Morally it should be the case that $\mathcal{L}_{p}^{\text {an }}\left(\operatorname{Sym}^{2}\left(\mathcal{F}_{k^{\prime}}\right)\left(k^{\prime}-1\right)\right)$ and $\mathcal{L}_{p}^{\text {an }}\left(\operatorname{Sym}^{2}\left(\mathcal{F}_{k}\right)(k-1)\right)$ are also congruent, albeit modulo a lesser power of $p$. Suppose that we want to compute the moments $\int x^{j} \cdot \mathrm{d} \mu_{\mathrm{Sym}^{2} \mathcal{F}_{k^{\prime}}\left(k^{\prime}-1\right)}^{\mathrm{imp},-}$ instead. Because $k^{\prime}=k+t(p-1) p^{r}$ with the chosen $r>1$, the strip $\left\{1, \ldots, k^{\prime}-1\right\}$ is considerable larger than the strip $\{1, \ldots, k-1\}$, so the range of $j$ 's for which $\operatorname{Hol}\left(\tilde{R}_{m, e}^{\left(k^{\prime}, j\right)}\right) \mid U_{p}^{2 m-1}$ is a classical weight $k^{\prime}$ modular form is now bigger. There are also more moments $\int x^{j} \cdot \mathrm{d} \mu_{\mathrm{Sym}^{2} \mathcal{F}_{k^{\prime}}\left(k^{\prime}-1\right)}^{\mathrm{imp},-}$ available. 
The main hindrance is that expressing $\operatorname{Hol}\left(\tilde{R}_{m, e}^{\left(k^{\prime}, j\right)}\right) \mid U_{p}^{2 m-1}$ in terms of a basis of weight $k^{\prime}$ modular forms is computationally far slower than before, as the dimension of $\mathcal{M}_{k^{\prime}}\left(\Gamma_{0}(N p)\right)$ grows rapidly with $k^{\prime}$. Therefore any advantage gained by calculating this larger set of moments is immediately offset by the slowness in writing each $\operatorname{Hol}\left(\tilde{R}_{m, e}^{\left(k^{\prime}, j\right)}\right) \mid U_{p}^{2 m-1}$ in terms of a $\mathbb{C}$-basis. For example, if $p=5, N=11, k=2$ and $k^{\prime}=2+(5-1) 5^{10}$ then a simple SAGE calculation reveals $\operatorname{dim}_{\mathbb{C}}\left(\mathcal{M}_{k^{\prime}}\left(\Gamma_{0}(N p)\right)\right)=234,375,008$, which is crippling from a numerical standpoint. Nevertheless, because the subspace of $p$-ordinary modular forms has fixed dimension by Hida's control theory, any theoretical result which could bypass the slowness in computing a full basis for $\mathcal{M}_{k^{\prime}}\left(\Gamma_{0}(N p)\right)$ would make the algorithm far more efficient.

\section{The Basic Method}

Using the SAGE computer package, we implemented the method outlined in $\S 2.1-\S 2.4$ to compile tables of $\left.\frac{\mathrm{d}}{\mathrm{d} s} \mathbf{L}_{p}^{\mathrm{imp}}\left(\operatorname{Sym}^{2} E, s\right)\right|_{s=1}$ for all curves $E$ of conductor $N_{E} \leq 300$ such that $4 \mid N_{E}$, as well as their symmetric square $\mathcal{L}$-invariants. These numerical values are tabulated in Appendix B. Here we were mainly interested in verifying that $\mathcal{L}_{p}^{\text {an }}\left(\mathrm{Sym}^{2} E\right)$ was non-zero, rather than in computing it to a high $p$-adic accuracy.

\subsection{An algorithm to compute the $\mathcal{L}$-invariant numerically}

We begin with some general observations. Assume we are given an elliptic curve $E_{/ \mathbb{Q}}$ with no restriction on its conductor $N_{E}$. Then $\mathcal{L}_{p}^{\text {an }}\left(\operatorname{Sym}^{2} E\right)$ depends only on the $\mathbb{Q}$-isogeny class of $E$. Indeed Nastasescu [29] has shown that the $p$-adic $L$-function for $\operatorname{Sym}^{2} E$ uniquely determines the $\mathbb{Q}$-isogeny class of the elliptic curve $E$, up to a twist by a quadratic character.

Let $l \neq 2$ be a prime. We write $\omega_{l}: \mathbb{F}_{l}^{\times} \rightarrow \mu_{l-1}$ for the Teichmüller character modulo $l$, which associates to each $x \in \mathbb{F}_{l}^{\times}$the unique $(l-1)$-st root of unity congruent to $x$ modulo $l$. One can then define a quadratic character $\varpi_{l}: \mathbb{F}_{l}^{\times} \rightarrow\{ \pm 1\}$ by the rule $\varpi_{l}(x)=\omega_{l}^{(l-1) / 2}(x)$. However if $l=2$, then $\varpi_{2}:(\mathbb{Z} / 4 \mathbb{Z})^{\times} \rightarrow\{ \pm 1\}$ denotes the quadratic character of conductor 4 .

Step 1: If $E$ has conductor $N_{E}$ divisible by 4 and $2 \leq \operatorname{ord}_{2}\left(N_{E \otimes \theta}\right)<\operatorname{ord}_{2}\left(N_{E}\right)$ where $\theta$ is one of $\varpi_{2},\left(\frac{\overline{2}}{2}\right)$, and $\left(\frac{-}{-2}\right)$, then replace $E$ with its twist $E \otimes \theta$; alternatively, if $E$ has conductor $N_{E}$ such that $\operatorname{ord}_{2}\left(N_{E}\right) \leq 1$, then replace $E$ with its twist $E \otimes \varpi_{2}$ to ensure that $4 \mid N_{E}$ holds.

Step 2: For our (possibly new) choice of $E$, let us define the set

$$
S_{1}=S_{1}(E):=\left\{\text { primes } l \mid N_{E} \text { such that } \operatorname{ord}_{l}\left(j_{E}\right) \geq 0\right\} .
$$

Note if the $j$-invariant of $E$ satisfies $\operatorname{ord}_{l}\left(j_{E}\right)<0$, then the Euler factor at $l$ for $D(E, s)$ equals $1-( \pm 1)^{2} l^{-s}=1-l^{-s}$ which agrees with that of $L_{\infty}\left(\mathrm{Sym}^{2} E, s\right)$, hence there is no discrepancy; the contribution to the $l$-part of the conductor is then given by $\operatorname{ord}_{l}\left(C_{\mathrm{Sym}^{2} E}\right)=2$ if $l \| N_{E}$.

We shall now compute the bad Euler factors, $H_{l}(X)$, at each prime number $l \in S_{1}$, and also the $l$-part of $C_{\mathrm{Sym}^{2} E}$ (we should point out that $E$ has potential good reduction at all $l \in S_{1}$ ). If $l \neq 2$, we write $\Phi_{l} \subset \operatorname{Gal}\left(\mathbb{Q}_{l}\left(E_{4}\right) / \mathbb{Q}_{l}\right)$ for the inertia subgroup; alternatively, if $l=2$ then $\Phi_{2} \subset \operatorname{Gal}\left(\mathbb{Q}_{2}\left(E_{3}\right) / \mathbb{Q}_{2}\right)$ will denote the inertia subgroup.

Step 3a: Suppose $E \otimes \theta$ has good reduction at $l$ where $\theta=\varpi_{l}$ if $l>2$, or $\theta \in\left\{\varpi_{2},\left(\frac{\overline{2}}{2}\right),\left(\frac{-}{-2}\right)\right\}$ if $l=2$; then $H_{l}(X)=\left(1-\hat{\alpha}_{l}^{2} X\right)\left(1-\hat{\beta}_{l}^{2} X\right)(1-l X)$ with $\Phi_{l} \cong C_{2}$ and $\operatorname{ord}_{l}\left(C_{\mathrm{Sym}^{2} E}\right)=0$, where the Hecke polynomial $1-a_{l}(E \otimes \theta) X+l X^{2}=\left(1-\hat{\alpha}_{l} X\right)\left(1-\hat{\beta}_{l} X\right)$. 
Step 3b: If $\# \Phi_{l}>2$, then each factor $H_{l}(X)$ is determined by Proposition A.1 in the Appendix, in which case $\operatorname{ord}_{l}\left(C_{\mathrm{Sym}^{2} E}\right)$ can be read off from the results in [7, pp120-121].

Step 4: Evaluate the imprimitive $L$-value $\xi_{\mathrm{Sym}^{2} E}=\frac{D(E, 1)}{\pi\left\langle f_{E}, f_{E}\right\rangle_{N_{E}}}$ using the formula in Lemma 2.1, which requires both $\prod_{l \in S_{1}} H_{l}(X)$ and $C_{\mathrm{Sym}^{2} E}=\prod_{l \mid N_{E}} l^{\operatorname{ord}_{l}\left(C_{\mathrm{Sym}^{2} E}\right)}$ from the previous steps. However, if $D(E, 1)=0$ then STOP!

Step 5: Compute $\mathbf{d}_{\mathcal{S}}$ and $\mathbf{d}_{\text {Eis }}$, then find a $q$-coefficient matrix $M=M(\mathfrak{N})$ with $\operatorname{det}(M) \neq 0$.

Step 6: Fix the desired accuracy $m \geq 1$; then for each $e \in\left(\mathbb{Z} / p^{m} \mathbb{Z}\right)^{\times}$, compute both of the terms $\delta_{1}(m, e)$ and $\delta_{2}(m, e)$ by following the method described at the end of Corollary 2.8.

Step 7: Calculate $\mathcal{L}_{p}^{\text {an }}\left(\operatorname{Sym}^{2} E\right) \bmod p^{m+\nu_{m, p}-\epsilon_{p}}$ via the numerical congruences in Theorem 2.10.

The structure of these inertia subgroups $\Phi_{l}$ was worked out completely by Serre in $[32, \S 5.6]$. To summarise, if $\operatorname{ord}_{l}\left(j_{E}\right) \geq 0$ and $l \mid N_{E}$ then $\Phi_{l} \in\left\{C_{2}, C_{3}, C_{4}, C_{6}\right\}$ provided that $l \neq 2,3$. If $l=3$ then the semi-direct product $C_{4} \rtimes C_{3}$ is also a possibility, while if $l=2$ then both $\mathrm{SL}_{2}\left(\mathbb{F}_{3}\right)$ and $Q_{8}$ (the quaternion group of size 8) can also occur as $\Phi_{l}$.

Fortunately, there is an extensive table given in [7, p121] which contains the information required to pin down the structure of $\Phi_{2}$ and $\Phi_{3}$, as well as the 2- and 3-parts of $C_{\mathrm{Sym}^{2} E}$. Therefore Step 3 can be fully automated.

We should also point out that the matrix $M(\mathfrak{N})$ in Step 5 need only be determined once, which is lucky because $\mathbf{d}_{\mathcal{S}}+\mathbf{d}_{\text {Eis }}$ can typically be greater than $10^{4}$ even if $N_{E}$ is relatively small.

\subsection{A general formula for $\mathbf{L}_{p}\left(\operatorname{Sym}^{2} E, 1\right)^{\prime}$ modulo $p^{m}$, even when $D(E, 1)=0$}

It is important to mention for the six elliptic curves 176b1, 196a1, 200b1, 240d1, 272b1, 300c1, the value of $\mathbf{L}_{p}^{\mathrm{imp}}\left(\mathrm{Sym}^{2} E, 1\right)^{\prime}$ is zero at all primes $p$ simply because $D(E, s)$ vanishes at $s=1$. One should note that the triviality of $\mathbf{L}_{p}^{\mathrm{imp}}\left(\mathrm{Sym}^{2} E, 1\right)^{\prime}$ does not imply either the triviality of $\mathbf{L}_{p}\left(\operatorname{Sym}^{2} E, 1\right)^{\prime}$, nor the triviality of $\mathcal{L}_{p}^{\text {an }}\left(\operatorname{Sym}^{2} E\right)$.

In order that our study of Conjecture 1.3 is not missing out any curves of conductor $\leq 300$, for those six elliptic curves listed above with $D(E, 1)=0$, we shall now describe a general method to approximate $\mathbf{L}_{p}\left(\operatorname{Sym}^{2} E, 1\right)^{\prime}$ that will work irrespective of whether $D(E, 1)$ is zero. Let us begin by partitioning the set $S_{1}=S_{1}(E)$ into a disjoint union of

$$
\begin{aligned}
& S_{1,-}^{\prime}:=\left\{l \in S_{1}-\{2\} \text { such that } \# \Phi_{l}>2 \text { and } \operatorname{Gal}\left(\mathbb{Q}_{l}\left(E_{4}\right) / \mathbb{Q}_{l}\right) \text { is abelian }\right\}, \\
& S_{1,-}^{\prime \prime}:=\left\{l \in S_{1} \text { such that } \# \Phi_{l}=2\right\}, \text { and } S_{1,+}:=S_{1}-S_{1,-}^{\prime}-S_{1,-}^{\prime \prime} .
\end{aligned}
$$

A careful reading of the argument in [7, pp119-121] indicates that for each prime $l \in S_{1,-}^{\prime} \cup S_{1,-}^{\prime \prime}$, one has $H_{l}(X)=(1-l X) \cdot \Upsilon_{l}(X)$ where

$$
\Upsilon_{l}(X):= \begin{cases}1 & \text { if } l \in S_{1,-}^{\prime} \\ \left(1-\hat{\alpha}_{l}^{2} X\right)\left(1-\hat{\beta}_{l}^{2} X\right) & \text { if } l \in S_{1,-}^{\prime \prime} .\end{cases}
$$

Alternatively, if a prime $l \in S_{1,+}$ then $H_{l}(X)=(1+l X)$ unless either $l=3$ and $\Phi_{3} \cong C_{4} \rtimes C_{3}$, or instead $l=2$ and $\Phi_{2} \in\left\{\mathrm{SL}_{2}\left(\mathbb{F}_{3}\right), Q_{8}\right\}$, in which case $H_{l}(X)=1$.

It follows that for $s \in \mathbb{C}$, there is a natural separation of Euler factors given by

$$
\prod_{l \in S_{1}} H_{l}\left(l^{-s}\right)=\left(\prod_{l \in S_{1,+}} H_{l}\left(l^{-s}\right) \times \prod_{l \in S_{1,-}^{\prime \prime}} \Upsilon_{l}\left(l^{-s}\right)\right) \times \prod_{l \in S_{1,-}^{\prime} \cup S_{1,-}^{\prime \prime}}\left(1-l^{1-s}\right)
$$

with the bracketted term non-zero at $s=1$, while the other term has order $\# S_{1,-}^{\prime}+\# S_{1,-}^{\prime \prime}$. 
Definition 3.1. For any $s \in \mathbb{Z}_{p}$, let us define the (period modified) p-adic L-function by

$$
\mathbf{L}_{p}^{\text {aut }}\left(\operatorname{Sym}^{2} E, s\right):=\frac{(2 \pi i)^{-1} \Omega_{E}^{+} \Omega_{E}^{-}}{\pi\left\langle f_{E}, f_{E}\right\rangle_{N_{E}}} \times \mathbf{L}_{p}\left(\operatorname{Sym}^{2} E, s\right) .
$$

Comparing the above with the imprimitive $p$-adic $L$-function, one can factorise the latter into

$$
\mathbf{L}_{p}^{\mathrm{imp}}\left(\operatorname{Sym}^{2} E, s\right)=\mathfrak{I}_{p}(s) \cdot \prod_{l \in S_{1,-}^{\prime} \cup S_{1,-}^{\prime \prime}}\left(1-<l>_{p}^{s-1}\right) \times \mathbf{L}_{p}^{\text {aut }}\left(\operatorname{Sym}^{2} E, s\right)
$$

where $\mathfrak{I}_{p}(s) \in \mathbb{Z}_{p}\langle\langle s\rangle\rangle$ is an Iwasawa function satisfying

$$
\Im_{p}(1)=\prod_{l \in S_{1,+}} H_{l}\left(l^{-1}\right) \times \prod_{l \in S_{1,-}^{\prime \prime}} \Upsilon_{l}\left(l^{-1}\right)
$$

It follows directly from this factorisation that

$$
\operatorname{order}_{s=1}\left(\mathbf{L}_{p}^{\mathrm{imp}}\left(\operatorname{Sym}^{2} E, s\right)\right)=\# S_{1,-}^{\prime}+\# S_{1,-}^{\prime \prime}+\operatorname{order}_{s=1}\left(\mathbf{L}_{p}^{\mathrm{aut}}\left(\operatorname{Sym}^{2} E, s\right)\right),
$$

hence Conjecture 1.3 is equivalent to $\mathbf{L}_{p}^{\mathrm{imp}}\left(\operatorname{Sym}^{2} E, s\right)$ vanishing with order $1+\# S_{1,-}^{\prime}+\# S_{1,-}^{\prime \prime}$ at the critical point $s=1$.

To verify Coates and Greenberg's conjecture for a given elliptic curve $E$ when $S_{1,-}^{\prime} \cup S_{1,-}^{\prime \prime} \neq \emptyset$, we must therefore supply a method to calculate $\mathbf{L}_{p}^{\text {aut }}\left(\operatorname{Sym}^{2} E, 1\right)^{\prime}$, then check it is non-zero.

Theorem 3.2. For all integers $m \geq 1$, there are congruences

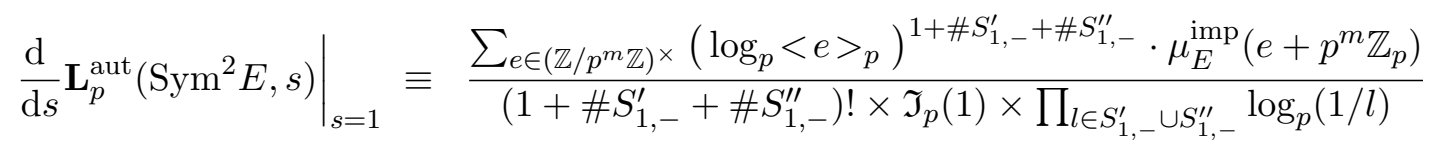

$$
\begin{aligned}
& \text { modulo } p^{m+\nu_{m, p}-\operatorname{ord}_{p}\left(\mathfrak{I}_{p}(1) \times \prod_{l \in S_{1,-}^{\prime} \cup S_{1,-}^{\prime \prime}} \log _{p}(1 / l)\right)} \text {. }
\end{aligned}
$$

Proof. Let us first set $\kappa_{p}:=\# S_{1,-}^{\prime}+\# S_{1,-}^{\prime \prime} \geq 0$. We have the following Taylor series at $s=1$ :

- $\quad \mathbf{L}_{p}^{\mathrm{imp}}\left(\operatorname{Sym}^{2} E, s\right)=\left.\frac{\mathrm{d}^{\kappa_{p}+1} \mathbf{L}_{p}^{\mathrm{imp}}\left(\operatorname{Sym}^{2} E, s\right)}{\mathrm{d} s^{\kappa_{p}+1}}\right|_{s=1} \cdot \frac{(s-1)^{\kappa_{p}+1}}{\left(\kappa_{p}+1\right) !}+O\left((s-1)^{\kappa_{p}+2}\right)$

- $\quad \mathbf{L}_{p}^{\text {aut }}\left(\operatorname{Sym}^{2} E, s\right)=\left.\frac{\mathrm{d}_{p}^{\text {aut }}\left(\operatorname{Sym}^{2} E, s\right)}{\mathrm{d} s}\right|_{s=1} \cdot(s-1)+O\left((s-1)^{2}\right)$

- $\quad \mathfrak{I}_{p}(s)=\mathfrak{I}_{p}(1) \cdot(s-1)^{0}+O\left((s-1)^{1}\right)$

- $\quad\left(1-<l>_{p}^{s-1}\right)=\log _{p}(1 / l) \cdot(s-1)^{1}+O\left((s-1)^{2}\right)$ for each prime $l \neq p$.

Plugging these directly into Equation $(9)$, one reads off from the $(s-1)^{\kappa_{p}+1}$-term that

$$
\left.\frac{\mathrm{d}_{p}^{\text {aut }}\left(\operatorname{Sym}^{2} E, s\right)}{\mathrm{d} s}\right|_{s=1}=\frac{\left.\frac{\mathrm{d}^{\kappa_{p}+1} \mathbf{L}_{p}^{\mathrm{imp}}\left(\mathrm{Sym}^{2} E, s\right)}{\mathrm{d} s^{\kappa} p+1}\right|_{s=1}}{\left(\kappa_{p}+1\right) ! \cdot \mathfrak{I}_{p}(1) \times \prod_{l \in S_{1,-}^{\prime} \cup S_{1,-}^{\prime \prime}} \log _{p}(1 / l)} .
$$

Further, upon differentiating the Mazur-Mellin transform $\left(\kappa_{p}+1\right)$-times, one easily deduces

$$
\begin{aligned}
\left.\frac{\mathrm{d}^{\kappa_{p}+1} \mathbf{L}_{p}^{\mathrm{imp}}\left(\operatorname{Sym}^{2} E, s\right)}{\mathrm{d} s^{\kappa_{p}+1}}\right|_{s=1} & =\int_{x \in \mathbb{Z}_{p}^{\times}}\left(\log _{p}<x>_{p}\right)^{\kappa_{p}+1} \cdot \mathrm{d} \mu_{E}^{\mathrm{imp}}(x) \\
& \equiv \sum_{e \in\left(\mathbb{Z} / p^{m} \mathbb{Z}\right)^{\times}}\left(\log _{p}<e>_{p}\right)^{\kappa_{p}+1} \times \mu_{E}^{\mathrm{imp}}\left(e+p^{m} \mathbb{Z}_{p}\right) \bmod p^{m+\nu_{p}} .
\end{aligned}
$$

The approximation now follows after dividing by $\left(\kappa_{p}+1\right) ! \cdot \mathfrak{I}_{p}(1) \times \prod_{l \in S_{1,-}^{\prime} \cup S_{1,-}^{\prime \prime}} \log _{p}(1 / l)$. 
Remarks: (a) The preceding theorem yields an effective method to calculate $\mathbf{L}_{p}^{\text {aut }}\left(\operatorname{Sym}^{2} E, 1\right)^{\prime}$, as a formula for the moments of the measure $\mathrm{d} \mu_{E}^{\mathrm{imp}}$ has already been given in Lemma 2.9.

(b) The $\mathcal{L}$-invariant itself is then obtained simply by working out the ratio

$$
\mathcal{L}_{p}^{\mathrm{an}}\left(\operatorname{Sym}^{2} E\right)=\left.\frac{\mathrm{d}}{\mathrm{d} s} \mathbf{L}_{p}^{\mathrm{aut}}\left(\operatorname{Sym}^{2} E, s\right)\right|_{s=1} \times\left(\left(1-\alpha_{p}^{-2}\right)\left(1-p \alpha_{p}^{-2}\right) \times \frac{L_{\infty}\left(\mathrm{Sym}^{2} E, 1\right)}{\pi\left\langle f_{E}, f_{E}\right\rangle_{N_{E}}}\right)^{-1} .
$$

(c) In the Appendix A, we give a method to determine $S_{1,+}, S_{1,-}^{\prime}, S_{1,-}^{\prime \prime}$ and also the $H_{l}(X)$ 's.

(d) If $S_{1,-}^{\prime}=S_{1,-}^{\prime \prime}=\emptyset$ so that $D(E, 1) \neq 0$, then Theorem 3.2 and the $\mathcal{L}$-invariant equation specialise to the situation covered in $\S 2.4-$ here $\mathbf{L}_{p}^{\text {aut }}$ and $\mathbf{L}_{p}^{\text {imp }}$ have the same order at $s=1$.

A worked example at level 176 . Consider the elliptic curve

$$
E=176 b 1: y^{2}=x^{3}+x^{2}-5 x-13
$$

of conductor $N_{E}=2^{4} \cdot 11$. Its first few good ordinary primes are $p=3,5,7,13, \ldots$ with corresponding Hecke eigenvalues $a_{3}(E)=1, a_{5}(E)=1, a_{7}(E)=2, a_{13}(E)=4, \ldots$ respectively. The quadratic twist $E \otimes \varpi_{2}$ has conductor 11 , and therefore will be $\mathbb{Q}$-isogenous to $X_{0}(11)$. One determines that $S_{1,+}(E)=S_{1,-}^{\prime}(E)=\emptyset$ and $S_{1,-}^{\prime \prime}(E)=\{2\}$, with

$$
\Upsilon_{2}(X)=\left(1-\hat{\alpha}_{2}^{2} X\right)\left(1-\hat{\beta}_{2}^{2} X\right)=1+4 X^{2}
$$

where $\hat{\alpha}_{2}=-1+i$ and $\hat{\beta}_{2}=-1-i$ are the roots of $X^{2}-a_{2}\left(X_{0}(11)\right) X+2=X^{2}+2 X+2$. Furthermore at $s=1$, the primitive complex $L$-function satisfies

$$
\frac{L_{\infty}\left(\mathrm{Sym}^{2} E, 1\right)}{\pi\left\langle f_{E \otimes \varpi_{2}}, f_{E \otimes \varpi_{2}}\right\rangle_{N_{E \otimes \varpi_{2}}}}=\frac{4 \cdot \sqrt{C_{\mathrm{Sym}^{2} E}}}{N_{E \otimes \varpi_{2}}} \times \prod_{l \in S_{1}\left(E \otimes \varpi_{2}\right)} \frac{1}{H_{l}\left(l^{-2}\right)}=\frac{4 \cdot \sqrt{121}}{11} \times 1=4 .
$$

The period ratio is given by

$$
\begin{aligned}
& \frac{\left\langle f_{E \otimes \varpi_{2}}, f_{E \otimes \varpi_{2}}\right\rangle_{N_{E \otimes \varpi_{2}}}}{\left\langle f_{E}, f_{E}\right\rangle_{N_{E}}}=\left[\Gamma_{0}\left(N_{E \otimes \varpi_{2}}\right): \Gamma_{0}\left(N_{E}\right)\right]^{-1} \times \operatorname{Res}_{s=2}\left(\frac{\sum_{n=1}^{\infty} a_{n}\left(E \otimes \varpi_{2}\right) \cdot n^{-s}}{\sum_{n=1}^{\infty} a_{n}(E) \cdot n^{-s}}\right) \\
& =\frac{N_{E \otimes \varpi_{2}} \cdot \prod_{l \mid N_{E \otimes \varpi_{2}}}(1+1 / l)}{N_{E} \cdot \prod_{l \mid N_{E}}(1+1 / l)} \times \frac{1+1 / 2}{D_{2}\left(E \otimes \varpi_{2}, 2\right)}=\frac{1}{16 \times\left(\left(1-2^{1-2}\right) \cdot \Upsilon_{2}\left(2^{-2}\right)\right)}=\frac{1}{10},
\end{aligned}
$$

in which case

$$
\frac{L_{\infty}\left(\mathrm{Sym}^{2} E, 1\right)}{\pi\left\langle f_{E}, f_{E}\right\rangle_{N_{E}}}=\frac{L_{\infty}\left(\mathrm{Sym}^{2} E, 1\right)}{\pi\left\langle f_{E \otimes \varpi_{2}}, f_{E \otimes \varpi_{2}}\right\rangle_{N_{E \otimes \varpi_{2}}}} \times \frac{\left\langle f_{E \otimes \varpi_{2}}, f_{E \otimes \varpi_{2}}\right\rangle_{N_{E \otimes \varpi_{2}}}}{\left\langle f_{E}, f_{E}\right\rangle_{N_{E}}}=4 \times \frac{1}{10}=\frac{2}{5} .
$$

Now for each choice of prime $p \in\{3,5,7,13\}$, applying Theorem 3.2 yields the congruences

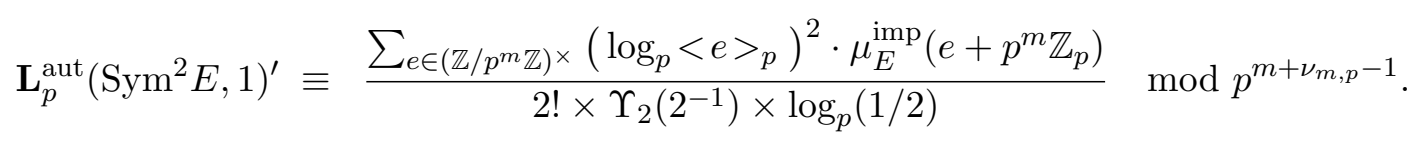

Evaluating the moments of the measure $\mathrm{d} \mu_{E}^{\mathrm{imp}}$ (via Lemma 2.9) for varying $m \geq 2$, we obtain

$$
\begin{array}{ll}
\mathbf{L}_{3}^{\text {aut }}\left(\operatorname{Sym}^{2} E, 1\right)^{\prime}=p+O\left(p^{4}\right), & \mathbf{L}_{5}^{\text {aut }}\left(\mathrm{Sym}^{2} E, 1\right)^{\prime}=p+O\left(p^{2}\right), \\
\mathbf{L}_{7}^{\text {aut }}\left(\operatorname{Sym}^{2} E, 1\right)^{\prime}=2 p+O\left(p^{2}\right), & \mathbf{L}_{13}^{\text {aut }}\left(\operatorname{Sym}^{2} E, 1\right)^{\prime}=4 p+O\left(p^{2}\right) .
\end{array}
$$


Finally, dividing the above derivatives by $\left(1-\alpha_{p}^{-2}\right)\left(1-p \alpha_{p}^{-2}\right) \times \frac{L_{\infty}\left(\mathrm{Sym}^{2} E, 1\right)}{\pi\left\langle f_{E}, f_{E}\right\rangle_{N_{E}}}$, we conclude that

$$
\begin{aligned}
& \mathcal{L}_{3}^{\text {an }}\left(\operatorname{Sym}^{2} X_{0}(11)\right)=\mathcal{L}_{3}^{\text {an }}\left(\operatorname{Sym}^{2} E\right)=1+2 p^{2}+O\left(p^{3}\right) \\
& \mathcal{L}_{5}^{\text {an }}\left(\operatorname{Sym}^{2} X_{0}(11)\right)=\mathcal{L}_{5}^{\text {an }}\left(\operatorname{Sym}^{2} E\right)=p+O\left(p^{2}\right) \\
& \mathcal{L}_{7}^{\text {an }}\left(\operatorname{Sym}^{2} X_{0}(11)\right)=\mathcal{L}_{7}^{\text {an }}\left(\operatorname{Sym}^{2} E\right)=2 p+O\left(p^{2}\right) \\
& \mathcal{L}_{13}^{\text {an }}\left(\operatorname{Sym}^{2} X_{0}(11)\right)=\mathcal{L}_{13}^{\text {an }}\left(\operatorname{Sym}^{2} E\right)=2 p+O\left(p^{2}\right)
\end{aligned}
$$

which are all non-zero elements of $\mathbb{Z}_{p}$.

Remark: In fact, if one chooses $p=11$ so that $X_{0}(11)$ has split multiplicative reduction at $p$, then it is established in [13, p51] that $\mathcal{L}_{11}^{\text {an }}\left(\operatorname{Sym}^{2} X_{0}(11)\right)=6 p+5 p^{2}+7 p^{3}+7 p^{4}+O\left(p^{5}\right) \neq 0$ by using an approach based on overconvergent modular symbols ${ }^{3}$. It follows immediately that Conjecture 1.3 must hold for the modular elliptic curve $X_{0}(11)$, at all odd primes $p<17$.

\subsection{The connection with deformation theory}

We conclude by interpreting these numerical calculations in the context of $\Lambda$-adic cusp forms. For a given elliptic curve $E_{/ \mathbb{Q}}$ and a good ordinary prime $p \geq 3$, one can lift the $p$-stabilisation $f_{0} \in \mathcal{S}_{2}\left(\Gamma_{0}\left(p N_{E}\right)\right)$ to an $\mathbb{I}$-adic eigenform, $\mathcal{F}$, where $\mathbb{I}$ denotes a suitable finite, flat extension of $\mathbb{Z}_{p} \llbracket X \rrbracket$, isomorphic to the irreducible component of the universal ordinary Hecke algebra carrying the form $f_{0}$.

For a sufficiently small choice of $p$-adic disk $\mathcal{W} \subset \mathbb{Z}_{p}$ centred on $k=2$, each specialisation

$$
\mathcal{F}_{k}:=\left.\mathcal{F}\right|_{X=(1+p)^{k-2}-1} \in \mathcal{S}_{k}\left(\Gamma_{0}\left(N_{E} p^{\infty}\right), \omega_{p}^{2-k}\right) \quad \text { for } k \in \mathcal{W} \cap \mathbb{Z}_{\geq 2}
$$

yields a classical cuspidal Hecke eigenform, with the $q$-expansion $\mathcal{F}_{k}(q)=\sum_{n=1}^{\infty} a\left(\mathcal{F}_{k}, n\right) q^{n}$. One can then interpolate each $q^{n}$-coefficient to yield a function, $a(\mathcal{F}(X), n)$, on the disk $\mathcal{W}$.

If $n=p$, then the derivative of $a(\mathcal{F}(X), p)$ with respect to $X$ is rigid meromorphic on $\mathcal{W}$. Hida established in [25, Prop 7.1] under suitable hypotheses (which are true, for instance, if the versal deformation ring $\mathcal{R}_{E}$ is Gorenstein) that $\frac{\mathrm{d} a(\mathcal{F}, p)}{\mathrm{d} X}$ is non-zero, and can thus vanish at only finitely many unspecified bad weights. Furthermore, the main formula in [26, Thm 1.1] yields

$$
\mathcal{L}_{p}^{\mathrm{Gr}}\left(\operatorname{Sym}^{2}\left(\mathcal{F}_{k}\right)(k)\right)=-2 \log _{p}(1+p) \cdot a\left(\mathcal{F}_{k}, p\right)^{-1} \times\left.\frac{\mathrm{d} a(\mathcal{F}, p)}{\mathrm{d} X}\right|_{X=(1+p)^{k-2}-1}
$$

for every weight $k \in \mathcal{W} \cap \mathbb{Z}_{\geq 2}$, where $\mathcal{L}_{p}^{\mathrm{Gr}}(-)$ again denotes Greenberg's algebraic $\mathcal{L}$-invariant.

Note that the Gorenstein property of the versal deformation ring $\mathcal{R}_{E}$ above has been verified for numerous elliptic curves $E$, and ordinary primes $p \geq 3$ (see $[4,24,35]$ ). For example, it is known to hold if the conductor $N_{E}$ of the elliptic curve is a square-free integer.

Remarks: (a) Let $\mathbf{L}_{p}\left(\mathcal{F}_{k} \otimes \mathcal{F}_{k}, s\right)$ denote the analytic $p$-adic $L$-function constructed in [23], which interpolates the special values $L\left(\mathcal{F}_{k} \otimes \mathcal{F}_{k} \otimes \chi, k\right)$. From Dasgupta's result in [10, Thm $1]$, one has a factorisation

$$
\mathbf{L}_{p}\left(\mathcal{F}_{k} \otimes \mathcal{F}_{k}, s\right)=\star \times \zeta_{p}\left(s-k+1, \omega_{p}^{0}\right) \times \mathbf{L}_{p}\left(\operatorname{Sym}^{2}\left(\mathcal{F}_{k}\right), s\right) .
$$

Here $\star$ consists of some Euler factors which are non-zero at classical weights, and so $\left.\star\right|_{s=k} \neq 0$.

\footnotetext{
${ }^{3}$ We also computed $\mathcal{L}_{p}\left(\operatorname{Sym}^{2} E\right)$ for $E=304 e 1$ at the good ordinary prime $p=5$, using an identical method. In fact $\mathcal{L}_{5}\left(\operatorname{Sym}^{2}(304 e 1)\right)=\mathcal{L}_{5}\left(\operatorname{Sym}^{2}(19 a 1)\right)$ because $E \otimes \varpi_{2}$ is $\mathbb{Q}$-isogenous to $19 a 1$; thankfully, the value we obtained numerically agreed with the 5 -adic expansion for $\mathcal{L}_{5}(19 a 1)$ given in [13, p52], at the weight $k+2=2$.
} 
(b) Allowing $s \rightarrow k$ and observing that $\operatorname{Res}_{s=k}\left(\zeta_{p}\left(s-k+1, \omega_{p}^{0}\right)\right)=1-p^{-1}$, the above implies

$$
\mathbf{L}_{p}\left(\mathcal{F}_{k} \otimes \mathcal{F}_{k}, k\right)=\mathcal{L}_{p}^{\text {an }}\left(\operatorname{Sym}^{2}\left(\mathcal{F}_{k}\right)(k)\right) \times \mathcal{E}_{p}\left(\mathcal{F}_{k}\right) \cdot \frac{L_{\infty}\left(\operatorname{Sym}^{2} \mathcal{F}_{k}, k\right)}{\Omega_{\infty, \operatorname{Sym}^{2}\left(\mathcal{F}_{k}\right)}}
$$

with $\mathcal{E}_{p}\left(\mathcal{F}_{k}\right)=\left.\star\right|_{s=k} \cdot\left(1-p^{-1}\right)\left(1-\alpha_{p}\left(\mathcal{F}_{k}\right)^{-2} p^{k-1}\right)\left(1-\beta\left(\mathcal{F}_{k}\right)_{p}^{-2} p^{-k}\right) \neq 0$.

(c) Under the same assumptions as [26, Thm 1.1], Citro proves in [5, Thm 1] that

$$
\mathbf{L}_{p}\left(\mathcal{F}_{k} \otimes \mathcal{F}_{k}, k\right)=\mathcal{L}_{p}^{\mathrm{Gr}}\left(\operatorname{Sym}^{2}\left(\mathcal{F}_{k}\right)(k)\right) \times \mathcal{E}_{p}\left(\mathcal{F}_{k}\right) \cdot \frac{L_{\infty}\left(\operatorname{Sym}^{2} \mathcal{F}_{k}, k\right)}{\Omega_{\infty, \operatorname{Sym}^{2}\left(\mathcal{F}_{k}\right)}} .
$$

Using Equations (11) and (12), Dasgupta [10, Thm 4] then reads off Greenberg's prediction that

$$
\mathcal{L}_{p}^{\mathrm{Gr}}\left(\operatorname{Sym}^{2}\left(\mathcal{F}_{k}\right)(k)\right)=\mathcal{L}_{p}^{\text {an }}\left(\operatorname{Sym}^{2}\left(\mathcal{F}_{k}\right)(k)\right)=\mathcal{L}_{p}^{\text {an }}\left(\operatorname{Sym}^{2}\left(\mathcal{F}_{k}\right)(k-1)\right)
$$

(note the second equality is a consequence of the $p$-adic functional equation for $\operatorname{Sym}^{2}\left(\mathcal{F}_{k}\right)$ ).

A corollary of these remarks is that we can replace the algebraic $\mathcal{L}$-invariant in Equation (10) with either analytic version. In particular, at weight two Hida's formula now becomes

$$
\left.\frac{\mathrm{d} a(\mathcal{F}, p)}{\mathrm{d} X}\right|_{X=0}=-\frac{\alpha_{p}}{2 \log _{p}(1+p)} \times \mathcal{L}_{p}^{\mathrm{an}}\left(\operatorname{Sym}^{2} E\right)
$$

hence the derivative of $a(\mathcal{F}, p)$ at zero coincides with $\frac{\mathcal{L}_{p}^{\text {an }}\left(\mathrm{Sym}^{2} E\right)}{p}$, up to an explicit $p$-adic unit. Of course, this could just end up being be the equation " $0=0$ " in disguise!

Nevertheless, combining our numerical calculations from Appendix B with Equation (13):

Corollary 3.3. Suppose that $E$ is an elliptic curve over $\mathbb{Q}$ of conductor $N_{E} \leq 300$ with $4 \mid N_{E}$, and let $p \leq 13$ be a prime of good ordinary reduction for $E$. Provided that $(E, p)$ is not one of the ten missing pairs listed in Theorem 1.4(ii)-(iii), it immediately follows that

$$
\left.\frac{\mathrm{d} a(\mathcal{F}, p)}{\mathrm{d} X}\right|_{X=0}=\delta_{p}(E) \cdot \frac{\mathcal{L}_{p}^{\mathrm{an}}\left(\mathrm{Sym}^{2} E\right)}{p} \neq 0
$$

where $\mathcal{F}$ denotes the Hida family lifting $f_{E} \in \mathcal{S}_{2}^{\text {new }}\left(\Gamma_{0}\left(N_{E}\right)\right)$, and $\delta_{p}(E):=-\frac{p \alpha_{p}}{2 \log _{p}(1+p)} \in \mathbb{Z}_{p}^{\times}$.

Dummit, Hablicsek, Harron, Jain, Pollack and Ross [13] have a direct method to calculate $a(\mathcal{F}, p)^{\prime}(0)$ through the use of overconvergent modular symbols, and they have computed four examples in op. cit., thereby establishing the non-triviality of $\mathcal{L}_{p}^{\mathrm{an}}\left(\mathrm{Sym}^{2} E\right)$ in these cases. Their results further determine power series expansions for $a\left(\mathcal{F}_{k}, p\right)$, as a function of $k$, over the weight-space $\mathcal{W}$.

The non-triviality of this $\mathcal{L}$-invariant has a key consequence for the Iwasawa Main Conjecture for $\operatorname{Sym}^{2} E$ over the cyclotomic $\mathbb{Z}_{p}$-extension $\mathbb{Q}^{\text {cyc }}$ of $\mathbb{Q}$. The property that $\mathcal{L}_{p}^{\text {an }}\left(\operatorname{Sym}^{2} E\right) \neq 0$ allows one to deduce that the order of the algebraic p-adic L-function at $s=1$ is exactly one. Here the algebraic $p$-adic $L$-function denotes the Mazur-Mellin transform of a generator, for the characteristic ideal of $\operatorname{Hom}_{\text {cont }}\left(\operatorname{Sel}_{p}\left(\operatorname{Sym}^{2} E(1) / \mathbb{Q}{ }^{\text {cyc }}\right), \mathbb{Q} / \mathbb{Z}\right)$ over the cyclotomic Iwasawa algebra $\mathbb{Z}_{p}\left[\left[\operatorname{Gal}\left(\mathbb{Q}^{\text {cyc }} / \mathbb{Q}\right)\right]\right]$ - we refer the reader to $[30$, Sect 10$]$ for a fuller discussion. 


\section{A Determining the set $S_{1}$, and the bad factors $H_{l}(X)$ with $l \in S_{1}$}

The purpose of this Appendix is to compute the decomposition $S_{1}=S_{1,+} \cup S_{1,-}^{\prime} \cup S_{1,-}^{\prime \prime}$, and the corresponding Euler factors $H_{l}(X)$. We retain the same notation and assumptions as $§ 3.2$. Let $\Delta_{E}$ denote the discriminant associated to a minimal Weierstrass equation for $E$ over $\mathbb{Z}$.

Proposition A.1. (a) A prime $l \in S_{1}$ belongs to the subset $S_{1,-}^{\prime \prime}$ if and only if $\operatorname{ord}_{l}\left(N_{E \otimes \theta}\right)=0$ at the character $\theta=\varpi_{l}$ if $l>2$, or instead at $\theta \in\left\{\varpi_{2},\left(\frac{\overline{2}}{2}\right),\left(\frac{-}{-2}\right)\right\}$ if $l=2$, in which case

$$
H_{l}(X)=\left(1-\hat{\alpha}_{l}^{2} X\right)\left(1-\hat{\beta}_{l}^{2} X\right)(1-l X)
$$

where $1-a_{l}(E \otimes \theta) X+l X^{2}=\left(1-\hat{\alpha}_{l} X\right)\left(1-\hat{\beta}_{l} X\right)$.

(b) A prime $l \in S_{1}-S_{1,-}^{\prime \prime}-\{2,3\}$ belongs to $S_{1,-}^{\prime}$ if and only if either

- $\operatorname{ord}_{l}\left(\Delta_{E}\right)=2,4,8,10$ and $l \equiv 1(\bmod 3)$, or

- $\operatorname{ord}_{l}\left(\Delta_{E}\right)=3,9$ and $l \equiv 1(\bmod 4)$

in which case $H_{l}(X)=1-l X$.

(c) A prime $l \in S_{1}-S_{1,-}^{\prime \prime}-\{2,3\}$ belongs to $S_{1,+}$ if and only if either

- $\operatorname{ord}_{l}\left(\Delta_{E}\right)=2,4,8,10$ and $l \equiv 2(\bmod 3)$, or

- $\operatorname{ord}_{l}\left(\Delta_{E}\right)=3,9$ and $l \equiv 3(\bmod 4)$

in which case $H_{l}(X)=1+l X$.

(d) For a prime $l \in\left(S_{1} \cap\{2,3\}\right)-S_{1,-}^{\prime \prime}$, one determines whether it belongs to $S_{1,-}^{\prime}$ or to $S_{1,+}$, and also its Euler factor $H_{l}(X)$, by using the tables in [7, p121] and Lemma 2.13 of op. cit.

Proof. Most of these statements follow from the description in [32] of the Galois representation $\rho_{E, p^{\infty}}: \operatorname{Gal}(\overline{\mathbb{Q}} / \mathbb{Q}) \rightarrow \mathrm{GL}_{2}\left(\mathbb{Z}_{p}\right)$ associated to the $p$-adic Tate module $\operatorname{Ta}_{p}(E):=\lim _{n} E_{p^{n}}$.

Firstly (a) is true because $\rho_{E, p^{\infty}} \otimes \theta$ will be unramified at $l$, and corresponds to the Tate module of the quadratic twist $E \otimes \theta$, which has good reduction at $l$ by the criterion of Néron, Ogg and Shafarevich; consequently $\operatorname{Sym}^{2}\left(\rho_{E, p^{\infty}}\right) \cong \operatorname{Sym}^{2}\left(\rho_{E, p^{\infty}} \otimes \theta\right)$ is also unramified at $l$.

To establish (b) and (c), let us now assume (i) the prime $l \geq 5$, and also (ii) $d_{l}:=\# \Phi_{l}>2$ so that $\Phi_{l} \in\left\{C_{3}, C_{4}, C_{6}\right\}$ here. Then using [7, Lemma 1.4],

$$
H_{l}(X)= \begin{cases}1-l X & \text { if } \mathbb{Q}_{l}\left(E_{p}\right) / \mathbb{Q}_{l} \text { is abelian } \\ 1+l X & \text { if } \mathbb{Q}_{l}\left(E_{p}\right) / \mathbb{Q}_{l} \text { is non-abelian. }\end{cases}
$$

Since $\mathbb{Q}_{l}\left(E_{p^{\infty}}\right) / \mathbb{Q}_{l}\left(E_{p}\right)$ is unramified, we observe that $\mathbb{Q}_{l}\left(E_{p}\right) / \mathbb{Q}_{l}$ is abelian if and only if $\mathbb{Q}_{l}\left(E_{p^{\infty}}\right) / \mathbb{Q}_{l}$ is abelian.

If $\mathbb{Q}_{l}\left(E_{p}\right) / \mathbb{Q}_{l}$ is abelian, then $\Phi_{l}$ factors through the inertia subgroup inside $\operatorname{Gal}\left(\mathbb{Q}_{l}^{\text {ab }} / \mathbb{Q}_{l}\right)$, and hence through $\operatorname{Gal}\left(\mathbb{Q}_{l}\left(\mu_{l \infty}\right) / \mathbb{Q}_{l}\right)$. Because $l \nmid d_{l}$ clearly $\operatorname{Gal}\left(\mathbb{Q}_{l}\left(\mu_{l} \infty\right) / \mathbb{Q}_{l}\left(\mu_{l}\right)\right)$ acts trivially on $\operatorname{Ta}_{p}(E)$, in which case $\Phi_{l}$ factors through $\operatorname{Gal}\left(\mathbb{Q}_{l}\left(\mu_{l}\right) / \mathbb{Q}_{l}\right)$, whence $l \equiv 1\left(\bmod d_{l}\right)$.

Conversely, there exists a unique tamely ramified extension $H_{d}$ of $\mathbb{Q}_{p}^{\text {nr }}$ with degree $d>0$. If $l \equiv 1\left(\bmod d_{l}\right)$ then the action of $\Phi_{l} \cong \rho_{E, p^{\infty}}\left(I_{l}\right)$ on $\operatorname{Ta}_{p}(E)$ factors through the algebraic extension $H_{d_{l}}=\mathbb{Q}_{l}^{\mathrm{nr}}\left(E_{p^{\infty}}\right) \subset \mathbb{Q}_{l}^{\mathrm{nr}}\left(\mu_{l}\right)$, which is certainly an abelian extension of $\mathbb{Q}_{l}$.

Conclusion: The extension $\mathbb{Q}_{l}\left(E_{p}\right) / \mathbb{Q}_{l}$ is abelian if and only if $l \equiv 1\left(\bmod d_{l}\right)$.

To complete the proof, we note that $d_{l}=\# \Phi_{l}$ can be read off from [32, p312] as follows:

- $\# \Phi_{l}=3$ if and only if $\operatorname{ord}_{l}\left(\Delta_{E}\right) \equiv 4$ or $8 \bmod 12$; 
- $\quad \# \Phi_{l}=4$ if and only if $\operatorname{ord}_{l}\left(\Delta_{E}\right) \equiv 3$ or $9 \bmod 12$;

- $\quad \# \Phi_{l}=6$ if and only if $\operatorname{ord}_{l}\left(\Delta_{E}\right) \equiv 2$ or $10 \bmod 12$.

It is then a tedious but straightforward exercise to verify that the conditions stated in (b) correspond to $l \equiv 1\left(\bmod d_{l}\right)$, while the conditions in $(\mathrm{c})$ correspond to $l \not \equiv 1\left(\bmod d_{l}\right)$.

\section{B Results for odd primes $p \leq 13$, in the range $11 \leq N_{E} \leq 300$}

Throughout we have only considered elliptic curves $E_{/ \mathbb{Q}}$ whose conductors are divisible by 4 . We first treat the curves with $D(E, 1) \neq 0$, and then the six exceptional curves with $D(E, 1)=0$.

\section{B.1 Tables of $\mathcal{L}$-invariants for elliptic curves $E$ with $D(E, 1) \neq 0$}

Tabulated below are the values we computed for both the derivative of $\mathbf{L}_{p}^{\mathrm{imp}}\left(\operatorname{Sym}^{2} E, s\right)$ at $s=1$ together with the corresponding $\mathcal{L}$-invariant term, for the elliptic curves $E$ with $D(E, 1) \neq 0$. If the elliptic curve $E$ is already a quadratic twist of another (earlier) elliptic curve listed in our tables, then we omit the $\mathcal{L}$-invariant data for $E$ completely.

The reader will notice for the elliptic curves of conductor 32 and 36, which have complex multiplication by $\mathbb{Q}(\sqrt{-1})$ and $\mathbb{Q}(\sqrt{-3})$ respectively, that $\mathcal{L}_{p}\left(\operatorname{Sym}^{2} E\right)$ coincides with $\log _{p}\left(\alpha_{p}^{-2}\right)$ in agreement with the Ferrero-Greenberg formula. However if $E$ has no complex multiplication, this identity no longer appears to hold in general.

$E=20 \mathrm{a} 1, C_{\mathrm{Sym}^{2} E}=10^{2}, S_{1}=\{2\}, \xi_{\mathrm{Sym}^{2} E}=\frac{8}{3}$
\begin{tabular}{|c|c|c|c|}
\hline$p$ & $a_{p}(E)$ & $\mathbf{L}_{p}^{\text {imp }}\left(\mathrm{Sym}^{2} E, 1\right)^{\prime}$ & $\mathcal{L}_{p}^{\text {an }}\left(\mathrm{Sym}^{2} E\right)$ \\
\hline 3 & -2 & $p^{2}+p^{3}+2 p^{4}+2 p^{5}+2 p^{6}+O\left(p^{7}\right)$ & $p^{2}+2 p^{4}+O\left(p^{7}\right)$ \\
\hline 7 & 2 & $2 p+2 p^{2}+p^{3}+O\left(p^{4}\right)$ & $p+2 p^{2}+p^{3}+O\left(p^{4}\right)$ \\
\hline 13 & 2 & $11 p+2 p^{2}+O\left(p^{3}\right)$ & $12 p+12 p^{2}+O\left(p^{3}\right)$ \\
\hline
\end{tabular}

$E=24 \mathrm{a} 1, C_{\mathrm{Sym}^{2} E}=12^{2}, S_{1}=\{2\}, \xi_{\mathrm{Sym}^{2} E}=2$
\begin{tabular}{|c|c|c|c|}
\hline$p$ & $a_{p}(E)$ & $\mathbf{L}_{p}^{\text {imp }}\left(\mathrm{Sym}^{2} E, 1\right)^{\prime}$ & $\mathcal{L}_{p}^{\text {an }}\left(\mathrm{Sym}^{2} E\right)$ \\
\hline 5 & -2 & $2 p^{2}+p^{3}+p^{4}+O\left(p^{5}\right)$ & $3 p^{2}+2 p^{3}+O\left(p^{5}\right)$ \\
\hline 11 & 4 & $6 p+8 p^{2}+O\left(p^{3}\right)$ & $p+O\left(p^{3}\right)$ \\
\hline 13 & -2 & $7 p+9 p^{2}+O\left(p^{3}\right)$ & $9 p+4 p^{2}+O\left(p^{3}\right)$ \\
\hline
\end{tabular}

$E=32 \mathrm{a} 1, C_{\mathrm{Sym}^{2} E}=8^{2}, S_{1}=\{2\}, \xi_{\mathrm{Sym}^{2} E}=1$

\begin{tabular}{|c|c|c|c|}
\hline$p$ & $a_{p}(E)$ & $\mathbf{L}_{p}^{\mathrm{imp}}\left(\mathrm{Sym}^{2} E, 1\right)^{\prime}$ & $\mathcal{L}_{p}^{\mathrm{an}}\left(\mathrm{Sym}^{2} E\right)$ \\
\hline 5 & -2 & $3 p+p^{2}+2 p^{3}+4 p^{4}+O\left(p^{5}\right)$ & $4 p+3 p^{2}+3 p^{3}+3 p^{4}+O\left(p^{5}\right)$ \\
\hline 13 & 6 & $p+9 p^{2}+O\left(p^{3}\right)$ & $4 p+7 p^{2}+O\left(p^{3}\right)$ \\
\hline
\end{tabular}

$E=36 \mathrm{a} 1, C_{\mathrm{Sym}^{2} E}=6^{2}, S_{1}=\{2,3\}, \xi_{\mathrm{Sym}^{2} E}=\frac{4}{3}$
\begin{tabular}{|c|c|c|c|}
\hline$p$ & $a_{p}(E)$ & $\mathbf{L}_{p}^{\mathrm{imp}}\left(\mathrm{Sym}^{2} E, 1\right)^{\prime}$ & $\mathcal{L}_{p}^{\mathrm{an}}\left(\mathrm{Sym}^{2} E\right)$ \\
\hline 7 & -4 & $6 p+3 p^{2}+2 p^{3}+O\left(p^{4}\right)$ & $2 p+3 p^{2}+2 p^{3}+O\left(p^{4}\right)$ \\
\hline 13 & 2 & $p^{2}+O\left(p^{3}\right)$ & $p^{2}+O\left(p^{3}\right)$ \\
\hline
\end{tabular}


$E=40 \mathrm{a} 1, C_{\mathrm{Sym}^{2} E}=20^{2}, S_{1}=\{2\}, \xi_{\mathrm{Sym}^{2} E}=2$

\begin{tabular}{|c|c|c|c|}
\hline$p$ & $a_{p}(E)$ & $\mathbf{L}_{p}^{\text {imp }}\left(\mathrm{Sym}^{2} E, 1\right)^{\prime}$ & $\mathcal{L}_{p}^{\text {an }}\left(\mathrm{Sym}^{2} E\right)$ \\
\hline 7 & -4 & $p+6 p^{3}+O\left(p^{4}\right)$ & $p+4 p^{2}+6 p^{3}+O\left(p^{4}\right)$ \\
\hline 11 & 4 & $5 p+4 p^{2}+O\left(p^{3}\right)$ & $10 p+3 p^{2}+O\left(p^{3}\right)$ \\
\hline 13 & -2 & $10 p+2 p^{2}+O\left(p^{3}\right)$ & $11 p+11 p^{2}+O\left(p^{3}\right)$ \\
\hline
\end{tabular}

$E=44 \mathrm{a} 1, C_{\mathrm{Sym}^{2} E}=22^{2}, S_{1}=\{2\}, \xi_{\mathrm{Sym}^{2} E}=\frac{8}{3}$

\begin{tabular}{|c|c|c|c|}
\hline$p$ & $a_{p}(E)$ & $\mathbf{L}_{p}^{\mathrm{imp}}\left(\mathrm{Sym}^{2} E, 1\right)^{\prime}$ & $\mathcal{L}_{p}^{\mathrm{an}}\left(\mathrm{Sym}^{2} E\right)$ \\
\hline 3 & 1 & $2 p^{3}+O\left(p^{6}\right)$ & $p^{3}+p^{5}+O\left(p^{6}\right)$ \\
\hline 5 & -3 & $4 p+2 p^{2}+p^{4}+O\left(p^{5}\right)$ & $2 p+3 p^{3}+3 p^{4}+O\left(p^{5}\right)$ \\
\hline 7 & 2 & $3 p+5 p^{3}+O\left(p^{4}\right)$ & $5 p+p^{2}+O\left(p^{4}\right)$ \\
\hline 13 & -4 & $3 p+p^{2}+O\left(p^{3}\right)$ & $9 p+11 p^{2}+O\left(p^{3}\right)$ \\
\hline
\end{tabular}

$E=52 \mathrm{a} 1, C_{\mathrm{Sym}^{2} E}=26^{2}, S_{1}=\{2\}, \xi_{\mathrm{Sym}^{2} E}=\frac{8}{3}$

\begin{tabular}{|c|c|c|c|}
\hline$p$ & $a_{p}(E)$ & $\mathbf{L}_{p}^{\text {imp }}\left(\mathrm{Sym}^{2} E, 1\right)^{\prime}$ & $\mathcal{L}_{p}^{\text {an }}\left(\mathrm{Sym}^{2} E\right)$ \\
\hline 5 & 2 & $4 p+2 p^{4}+O\left(p^{5}\right)$ & $2 p+2 p^{3}+3 p^{4}+O\left(p^{5}\right)$ \\
\hline 7 & -2 & $p^{3}+O\left(p^{4}\right)$ & $4 p^{3}+O\left(p^{4}\right)$ \\
\hline 11 & -2 & $10 p+p^{2}+O\left(p^{3}\right)$ & $5 p+9 p^{2}+O\left(p^{3}\right)$ \\
\hline
\end{tabular}

$E=56 \mathrm{a} 1, C_{\mathrm{Sym}^{2} E}=28^{2}, S_{1}=\{2\}, \xi_{\mathrm{Sym}^{2} E}=2$

\begin{tabular}{|c|c|c|c|}
\hline$p$ & $a_{p}(E)$ & $\mathbf{L}_{p}^{\mathrm{imp}}\left(\mathrm{Sym}^{2} E, 1\right)^{\prime}$ & $\mathcal{L}_{p}^{\text {an }}\left(\mathrm{Sym}^{2} E\right)$ \\
\hline 5 & 2 & $p+p^{2}+2 p^{3}+O\left(p^{4}\right)$ & $4 p+2 p^{3}+O\left(p^{4}\right)$ \\
\hline 11 & -4 & $p+O\left(p^{3}\right)$ & $2 p+10 p^{2}+O\left(p^{3}\right)$ \\
\hline 13 & 2 & $9 p+O\left(p^{2}\right)$ & $6 p+O\left(p^{2}\right)$ \\
\hline
\end{tabular}

$E=56 \mathrm{~b} 1, C_{\mathrm{Sym}^{2} E}=28^{2}, S_{1}=\{2\}, \xi_{\mathrm{Sym}^{2} E}=2$

\begin{tabular}{|c|c|c|c|}
\hline$p$ & $a_{p}(E)$ & $\mathbf{L}_{p}^{\text {imp }}\left(\mathrm{Sym}^{2} E, 1\right)^{\prime}$ & $\mathcal{L}_{p}^{\text {an }}\left(\mathrm{Sym}^{2} E\right)$ \\
\hline 3 & 2 & $2 p+p^{4}+O\left(p^{5}\right)$ & $2+p^{3}+O\left(p^{4}\right)$ \\
\hline 5 & -4 & $3 p+p^{2}+O\left(p^{3}\right)$ & $4+2 p+O\left(p^{2}\right)$ \\
\hline
\end{tabular}

$E=76 \mathrm{a} 1, C_{\mathrm{Sym}^{2} E}=38^{2}, S_{1}=\{2\}, \xi_{\mathrm{Sym}^{2} E}=\frac{8}{3}$

\begin{tabular}{|c|c|c|c|}
\hline$p$ & $a_{p}(E)$ & $\mathbf{L}_{p}^{\mathrm{imp}}\left(\mathrm{Sym}^{2} E, 1\right)^{\prime}$ & $\mathcal{L}_{p}^{\text {an }}\left(\mathrm{Sym}^{2} E\right)$ \\
\hline 3 & 2 & $1+2 p+O\left(p^{4}\right)$ & $1+p+2 p^{2}+O\left(p^{4}\right)$ \\
\hline 5 & -1 & $4 p+3 p^{2}+O\left(p^{3}\right)$ & $3+2 p+O\left(p^{2}\right)$ \\
\hline 7 & -3 & $p^{2}+O\left(p^{3}\right)$ & $6 p^{2}+O\left(p^{3}\right)$ \\
\hline 11 & 5 & $5 p+4 p^{2}+O\left(p^{3}\right)$ & $9 p+4 p^{2}+O\left(p^{3}\right)$ \\
\hline 13 & -4 & $4 p+9 p^{2}+O\left(p^{3}\right)$ & $12 p+3 p^{2}+O\left(p^{3}\right)$ \\
\hline
\end{tabular}

$E=84 \mathrm{a} 1, C_{\mathrm{Sym}^{2} E}=42^{2}, S_{1}=\{2\}, \xi_{\mathrm{Sym}^{2} E}=\frac{8}{3}$

\begin{tabular}{|c|c|c|c|}
\hline$p$ & $a_{p}(E)$ & $\mathbf{L}_{p}^{\text {imp }}\left(\mathrm{Sym}^{2} E, 1\right)^{\prime}$ & $\mathcal{L}_{p}^{\text {an }}\left(\mathrm{Sym}^{2} E\right)$ \\
\hline 11 & -6 & $5 p+3 p^{2}+O\left(p^{3}\right)$ & $9 p+4 p^{2}+O\left(p^{3}\right)$ \\
\hline 13 & 2 & $10 p+10 p^{2}+O\left(p^{3}\right)$ & $5 p+6 p^{2}+O\left(p^{3}\right)$ \\
\hline
\end{tabular}


$E=84 \mathrm{~b} 1, C_{\mathrm{Sym}^{2} E}=42^{2}, S_{1}=\{2\}, \xi_{\mathrm{Sym}^{2} E}=\frac{8}{3}$

\begin{tabular}{|c|c|c|c|}
\hline$p$ & $a_{p}(E)$ & $\mathbf{L}_{p}^{\text {imp }}\left(\mathrm{Sym}^{2} E, 1\right)^{\prime}$ & $\mathcal{L}_{p}^{\text {an }}\left(\mathrm{Sym}^{2} E\right)$ \\
\hline 5 & 4 & $p+2 p^{2}+O\left(p^{3}\right)$ & $1+3 p+O\left(p^{2}\right)$ \\
\hline 11 & 2 & $5 p+4 p^{2}+O\left(p^{3}\right)$ & $8 p+3 p^{2}+O\left(p^{3}\right)$ \\
\hline 13 & -6 & $7 p+3 p^{2}+O\left(p^{3}\right)$ & $4 p+2 p^{2}+O\left(p^{3}\right)$ \\
\hline
\end{tabular}

$E=88 \mathrm{a} 1, C_{\mathrm{Sym}^{2} E}=44^{2}, S_{1}=\{2\}, \xi_{\mathrm{Sym}^{2} E}=2$

\begin{tabular}{|c|c|c|c|}
\hline$p$ & $a_{p}(E)$ & $\mathbf{L}_{p}^{\text {imp }}\left(\mathrm{Sym}^{2} E, 1\right)^{\prime}$ & $\mathcal{L}_{p}^{\text {an }}\left(\mathrm{Sym}^{2} E\right)$ \\
\hline 5 & -3 & $4 p+p^{3}+O\left(p^{4}\right)$ & $p+4 p^{2}+p^{3}+O\left(p^{4}\right)$ \\
\hline 7 & -2 & $6 p+5 p^{2}+O\left(p^{3}\right)$ & $4 p+5 p^{2}+O\left(p^{3}\right)$ \\
\hline
\end{tabular}

$E=92 \mathrm{a} 1, C_{\mathrm{Sym}^{2} E}=46^{2}, S_{1}=\{2\}, \xi_{\mathrm{Sym}^{2} E}=\frac{8}{3}$

\begin{tabular}{|c|c|c|c|}
\hline$p$ & $a_{p}(E)$ & $\mathbf{L}_{p}^{\text {imp }}\left(\mathrm{Sym}^{2} E, 1\right)^{\prime}$ & $\mathcal{L}_{p}^{\text {an }}\left(\mathrm{Sym}^{2} E\right)$ \\
\hline 3 & 1 & $p^{4}+2 p^{5}+O\left(p^{6}\right)$ & $2 p^{4}+2 p^{5}+O\left(p^{6}\right)$ \\
\hline 7 & 2 & $6 p+2 p^{2}+O\left(p^{3}\right)$ & $3 p+4 p^{2}+O\left(p^{3}\right)$ \\
\hline 13 & -1 & $11 p+O\left(p^{2}\right)$ & $2+O(p)$ \\
\hline
\end{tabular}

$E=92 \mathrm{~b} 1, C_{\mathrm{Sym}^{2} E}=46^{2}, S_{1}=\{2\}, \xi_{\mathrm{Sym}^{2} E}=\frac{8}{3}$

\begin{tabular}{|c|c|c|c|}
\hline$p$ & $a_{p}(E)$ & $\mathbf{L}_{p}^{\mathrm{imp}}\left(\mathrm{Sym}^{2} E, 1\right)^{\prime}$ & $\mathcal{L}_{p}^{\text {an }}\left(\mathrm{Sym}^{2} E\right)$ \\
\hline 5 & -2 & $4 p+p^{2}+2 p^{3}+O\left(p^{4}\right)$ & $2 p+3 p^{2}+O\left(p^{4}\right)$ \\
\hline 7 & -4 & $3 p+3 p^{2}+O\left(p^{3}\right)$ & $4 p+4 p^{2}+O\left(p^{3}\right)$ \\
\hline 11 & 2 & $10 p+O\left(p^{2}\right)$ & $5 p+O\left(p^{2}\right)$ \\
\hline 13 & -5 & $12 p+O\left(p^{2}\right)$ & $12 p+O\left(p^{2}\right)$ \\
\hline
\end{tabular}

$E=96 \mathrm{a} 1, C_{\mathrm{Sym}^{2} E}=24^{2}, S_{1}=\{2\}, \xi_{\mathrm{Sym}^{2} E}=1$

\begin{tabular}{|c|c|c|c|}
\hline$p$ & $a_{p}(E)$ & $\mathbf{L}_{p}^{\text {imp }}\left(\mathrm{Sym}^{2} E, 1\right)^{\prime}$ & $\mathcal{L}_{p}^{\text {an }}\left(\mathrm{Sym}^{2} E\right)$ \\
\hline 5 & 2 & $p^{2}+2 p^{3}+O\left(p^{4}\right)$ & $3 p^{2}+4 p^{3}+O\left(p^{4}\right)$ \\
\hline 7 & -4 & $4 p+2 p^{2}+O\left(p^{3}\right)$ & $p+2 p^{2}+O\left(p^{3}\right)$ \\
\hline 11 & 4 & $9 p+O\left(p^{2}\right)$ & $3 p+O\left(p^{2}\right)$ \\
\hline 13 & -2 & $3 p+O\left(p^{2}\right)$ & $4 p+O\left(p^{2}\right)$ \\
\hline
\end{tabular}

$E=104 \mathrm{a} 1, C_{\mathrm{Sym}^{2} E}=52^{2}, S_{1}=\{2\}, \xi_{\mathrm{Sym}^{2} E}=2$

\begin{tabular}{|c|c|c|c|}
\hline$p$ & $a_{p}(E)$ & $\mathbf{L}_{p}^{\mathrm{imp}}\left(\mathrm{Sym}^{2} E, 1\right)^{\prime}$ & $\mathcal{L}_{p}^{\mathrm{an}}\left(\mathrm{Sym}^{2} E\right)$ \\
\hline 3 & 1 & $p+p^{2}+2 p^{3}+p^{4}+O\left(p^{5}\right)$ & $2+2 p+2 p^{3}+O\left(p^{4}\right)$ \\
\hline 5 & -1 & $2 p^{2}+O\left(p^{3}\right)$ & $2 p+O\left(p^{2}\right)$ \\
\hline 7 & 5 & $p+O\left(p^{3}\right)$ & $3 p+6 p^{2}+O\left(p^{3}\right)$ \\
\hline 11 & -2 & $9 p+O\left(p^{2}\right)$ & $6 p+O\left(p^{2}\right)$ \\
\hline
\end{tabular}

$E=108 \mathrm{a} 1, C_{\mathrm{Sym}^{2} E}=18^{2}, S_{1}=\{2,3\}, \xi_{\mathrm{Sym}^{2} E}=\frac{8}{9}$

\begin{tabular}{|c|c|c|c|}
\hline$p$ & $a_{p}(E)$ & $\mathbf{L}_{p}^{\text {imp }}\left(\mathrm{Sym}^{2} E, 1\right)^{\prime}$ & $\mathcal{L}_{p}^{\text {an }}\left(\mathrm{Sym}^{2} E\right)$ \\
\hline 7 & 5 & $6 p+5 p^{2}+O\left(p^{3}\right)$ & $2 p+3 p^{2}+O\left(p^{3}\right)$ \\
\hline 13 & -7 & $6 p^{2}+O\left(p^{3}\right)$ & $p^{2}+O\left(p^{3}\right)$ \\
\hline
\end{tabular}




$E=112 \mathrm{c} 1, C_{\mathrm{Sym}^{2} E}=14^{2}, S_{1}=\{\}, \xi_{\mathrm{Sym}^{2} E}=\frac{1}{2}$
\begin{tabular}{|c|c|c|c|}
\hline$p$ & $a_{p}(E)$ & $\mathbf{L}_{p}^{\text {imp }}\left(\mathrm{Sym}^{2} E, 1\right)^{\prime}$ & $\mathcal{L}_{p}^{\text {an }}\left(\mathrm{Sym}^{2} E\right)$ \\
\hline 3 & 2 & $p+2 p^{2}+O\left(p^{5}\right)$ & $1+p^{3}+O\left(p^{4}\right)$ \\
\hline 13 & -4 & $9 p+O\left(p^{2}\right)$ & $p+O\left(p^{2}\right)$ \\
\hline
\end{tabular}

$E=116 \mathrm{a} 1, C_{\mathrm{Sym}^{2} E}=58^{2}, S_{1}=\{2\}, \xi_{\mathrm{Sym}^{2} E}=\frac{8}{3}$

\begin{tabular}{|c|c|c|c|}
\hline$p$ & $a_{p}(E)$ & $\mathbf{L}_{p}^{\text {imp }}\left(\mathrm{Sym}^{2} E, 1\right)^{\prime}$ & $\mathcal{L}_{p}^{\text {an }}\left(\mathrm{Sym}^{2} E\right)$ \\
\hline 5 & 3 & $p+3 p^{2}+O\left(p^{3}\right)$ & $3 p+2 p^{2}+O\left(p^{3}\right)$ \\
\hline 7 & 4 & $4 p+3 p^{2}+O\left(p^{3}\right)$ & $3 p+2 p^{2}+O\left(p^{3}\right)$ \\
\hline 11 & -1 & $O\left(p^{2}\right)$ & $O(p)$ \\
\hline 13 & -3 & $12 p+O\left(p^{2}\right)$ & $p+O\left(p^{2}\right)$ \\
\hline
\end{tabular}

$E=116 \mathrm{~b} 1, C_{\mathrm{Sym}^{2} E}=58^{2}, S_{1}=\{2\}, \xi_{\mathrm{Sym}^{2} E}=\frac{8}{3}$

\begin{tabular}{|c|c|c|c|}
\hline$p$ & $a_{p}(E)$ & $\mathbf{L}_{p}^{\mathrm{imp}}\left(\mathrm{Sym}^{2} E, 1\right)^{\prime}$ & $\mathcal{L}_{p}^{\mathrm{an}}\left(\mathrm{Sym}^{2} E\right)$ \\
\hline 3 & 1 & $2 p^{2}+2 p^{3}+O\left(p^{5}\right)$ & $p^{2}+p^{3}+p^{4}+O\left(p^{5}\right)$ \\
\hline 5 & 3 & $3 p+4 p^{2}+4 p^{3}+O\left(p^{4}\right)$ & $4 p+2 p^{2}+4 p^{3}+O\left(p^{4}\right)$ \\
\hline 7 & -4 & $6 p+4 p^{2}+O\left(p^{3}\right)$ & $p+4 p^{2}+O\left(p^{3}\right)$ \\
\hline 11 & 3 & $5 p+O\left(p^{2}\right)$ & $4 p+O\left(p^{2}\right)$ \\
\hline 13 & 5 & $9 p+O\left(p^{2}\right)$ & $9 p+O\left(p^{2}\right)$ \\
\hline
\end{tabular}

$E=116 \mathrm{c} 1, C_{\mathrm{Sym}^{2} E}=58^{2}, S_{1}=\{2\}, \xi_{\mathrm{Sym}^{2} E}=\frac{8}{3}$

\begin{tabular}{|c|c|c|c|}
\hline$p$ & $a_{p}(E)$ & $\mathbf{L}_{p}^{\text {imp }}\left(\mathrm{Sym}^{2} E, 1\right)^{\prime}$ & $\mathcal{L}_{p}^{\text {an }}\left(\mathrm{Sym}^{2} E\right)$ \\
\hline 3 & 2 & $1+2 p+p^{2}+2 p^{3}+O\left(p^{4}\right)$ & $1+p+2 p^{3}+O\left(p^{4}\right)$ \\
\hline 5 & -2 & $p+4 p^{2}+O\left(p^{3}\right)$ & $3 p+4 p^{2}+O\left(p^{3}\right)$ \\
\hline 7 & 4 & $3 p+4 p^{2}+O\left(p^{3}\right)$ & $4 p+3 p^{2}+O\left(p^{3}\right)$ \\
\hline 11 & -6 & $2 p+O\left(p^{2}\right)$ & $8 p+O\left(p^{2}\right)$ \\
\hline 13 & 2 & $8 p+O\left(p^{2}\right)$ & $4 p+O\left(p^{2}\right)$ \\
\hline
\end{tabular}

$E=120 \mathrm{a} 1, C_{\mathrm{Sym}^{2} E}=60^{2}, S_{1}=\{2\}, \xi_{\mathrm{Sym}^{2} E}=2$

\begin{tabular}{|c|c|c|c|}
\hline$p$ & $a_{p}(E)$ & $\mathbf{L}_{p}^{\text {imp }}\left(\mathrm{Sym}^{2} E, 1\right)^{\prime}$ & $\mathcal{L}_{p}^{\text {an }}\left(\mathrm{Sym}^{2} E\right)$ \\
\hline 11 & -4 & $6 p+O\left(p^{2}\right)$ & $p+O\left(p^{2}\right)$ \\
\hline 13 & 6 & $11 p+O\left(p^{2}\right)$ & $9 p+O\left(p^{2}\right)$ \\
\hline
\end{tabular}

$E=120 \mathrm{~b} 1, C_{\mathrm{Sym}^{2} E}=60^{2}, S_{1}=\{2\}, \xi_{\mathrm{Sym}^{2} E}=2$

\begin{tabular}{|c|c|c|c|}
\hline$p$ & $a_{p}(E)$ & $\mathbf{L}_{p}^{\text {imp }}\left(\mathrm{Sym}^{2} E, 1\right)^{\prime}$ & $\mathcal{L}_{p}^{\text {an }}\left(\mathrm{Sym}^{2} E\right)$ \\
\hline 7 & 4 & $5 p+3 p^{2}+O\left(p^{3}\right)$ & $5 p+2 p^{2}+O\left(p^{3}\right)$ \\
\hline 13 & -6 & $6 p+O\left(p^{2}\right)$ & $12 p+O\left(p^{2}\right)$ \\
\hline
\end{tabular}


$E=124 \mathrm{a} 1, C_{\mathrm{Sym}^{2} E}=62^{2}, S_{1}=\{2\}, \xi_{\mathrm{Sym}^{2} E}=\frac{8}{3}$

\begin{tabular}{|c|c|c|c|}
\hline$p$ & $a_{p}(E)$ & $\mathbf{L}_{p}^{\text {imp }}\left(\mathrm{Sym}^{2} E, 1\right)^{\prime}$ & $\mathcal{L}_{p}^{\text {an }}\left(\mathrm{Sym}^{2} E\right)$ \\
\hline 3 & -2 & $2+2 p+p^{2}+O\left(p^{4}\right)$ & $2+p^{2}+2 p^{3}+O\left(p^{4}\right)$ \\
\hline 5 & -3 & $2 p+3 p^{2}+2 p^{3}+O\left(p^{4}\right)$ & $p+p^{2}+2 p^{3}+O\left(p^{4}\right)$ \\
\hline 7 & -1 & $2 p^{2}+O\left(p^{3}\right)$ & $4 p+O\left(p^{2}\right)$ \\
\hline 11 & -6 & $7 p^{2}+O\left(p^{3}\right)$ & $6 p^{2}+O\left(p^{3}\right)$ \\
\hline 13 & 2 & $4 p+O\left(p^{2}\right)$ & $2 p+O\left(p^{2}\right)$ \\
\hline
\end{tabular}

$E=124 \mathrm{~b} 1, C_{\mathrm{Sym}^{2} E}=62^{2}, S_{1}=\{2\}, \xi_{\mathrm{Sym}^{2} E}=\frac{8}{3}$

\begin{tabular}{|c|c|c|c|}
\hline$p$ & $a_{p}(E)$ & $\mathbf{L}_{p}^{\mathrm{imp}}\left(\mathrm{Sym}^{2} E, 1\right)^{\prime}$ & $\mathcal{L}_{p}^{\mathrm{an}}\left(\mathrm{Sym}^{2} E\right)$ \\
\hline 5 & 1 & $p+3 p^{2}+O\left(p^{3}\right)$ & $2+p+O\left(p^{2}\right)$ \\
\hline 7 & 3 & $5 p+6 p^{2}+O\left(p^{3}\right)$ & $2 p+5 p^{2}+O\left(p^{3}\right)$ \\
\hline 11 & 6 & $O\left(p^{3}\right)$ & $O\left(p^{3}\right)$ \\
\hline 13 & -4 & $11 p+O\left(p^{2}\right)$ & $7 p+O\left(p^{2}\right)$ \\
\hline
\end{tabular}

$E=128 \mathrm{a} 1, C_{\mathrm{Sym}^{2} E}=16^{2}, S_{1}=\{2\}, \xi_{\mathrm{Sym}^{2} E}=\frac{1}{2}$

\begin{tabular}{|c|c|c|c|}
\hline$p$ & $a_{p}(E)$ & $\mathbf{L}_{p}^{\text {imp }}\left(\mathrm{Sym}^{2} E, 1\right)^{\prime}$ & $\mathcal{L}_{p}^{\text {an }}\left(\mathrm{Sym}^{2} E\right)$ \\
\hline 3 & -2 & $2 p+2 p^{2}+p^{3}+O\left(p^{5}\right)$ & $2+p+p^{2}+2 p^{3}+O\left(p^{4}\right)$ \\
\hline 5 & -2 & $4 p+2 p^{2}+p^{3}+O\left(p^{4}\right)$ & $4 p+3 p^{3}+O\left(p^{4}\right)$ \\
\hline 7 & -4 & $3 p+5 p^{2}+O\left(p^{3}\right)$ & $5 p+6 p^{2}+O\left(p^{3}\right)$ \\
\hline 11 & 2 & $7 p+O\left(p^{2}\right)$ & $4 p+O\left(p^{2}\right)$ \\
\hline 13 & -2 & $5 p+O\left(p^{2}\right)$ & $9 p+O\left(p^{2}\right)$ \\
\hline
\end{tabular}

$E=132 \mathrm{a} 1, C_{\mathrm{Sym}^{2} E}=66^{2}, S_{1}=\{2\}, \xi_{\mathrm{Sym}^{2} E}=\frac{8}{3}$

\begin{tabular}{|c|c|c|c|}
\hline$p$ & $a_{p}(E)$ & $\mathbf{L}_{p}^{\text {imp }}\left(\mathrm{Sym}^{2} E, 1\right)^{\prime}$ & $\mathcal{L}_{p}^{\text {an }}\left(\mathrm{Sym}^{2} E\right)$ \\
\hline 5 & 2 & $4 p+p^{2}+p^{3}+O\left(p^{4}\right)$ & $2 p+3 p^{2}+2 p^{3}+O\left(p^{4}\right)$ \\
\hline 7 & -2 & $p+O\left(p^{3}\right)$ & $4 p+O\left(p^{3}\right)$ \\
\hline 13 & -2 & $12 p+O\left(p^{2}\right)$ & $6 p+O\left(p^{2}\right)$ \\
\hline
\end{tabular}

$E=132 \mathrm{~b} 1, C_{\mathrm{Sym}^{2} E}=66^{2}, S_{1}=\{2\}, \xi_{\mathrm{Sym}^{2} E}=\frac{8}{3}$

\begin{tabular}{|c|c|c|c|}
\hline$p$ & $a_{p}(E)$ & $\mathbf{L}_{p}^{\text {imp }}\left(\mathrm{Sym}^{2} E, 1\right)^{\prime}$ & $\mathcal{L}_{p}^{\text {an }}\left(\mathrm{Sym}^{2} E\right)$ \\
\hline 5 & 2 & $2 p+3 p^{2}+O\left(p^{3}\right)$ & $p+4 p^{2}+O\left(p^{3}\right)$ \\
\hline 7 & 2 & $3 p+p^{2}+O\left(p^{3}\right)$ & $5 p+5 p^{2}+O\left(p^{3}\right)$ \\
\hline 13 & 6 & $3 p+O\left(p^{2}\right)$ & $11 p+O\left(p^{2}\right)$ \\
\hline
\end{tabular}

$E=136 \mathrm{a} 1, C_{\mathrm{Sym}^{2} E}=68^{2}, S_{1}=\{2\}, \xi_{\mathrm{Sym}^{2} E}=2$

\begin{tabular}{|c|c|c|c|}
\hline$p$ & $a_{p}(E)$ & $\mathbf{L}_{p}^{\mathrm{imp}}\left(\mathrm{Sym}^{2} E, 1\right)^{\prime}$ & $\mathcal{L}_{p}^{\mathrm{an}}\left(\mathrm{Sym}^{2} E\right)$ \\
\hline 3 & -2 & $2 p^{2}+2 p^{3}+p^{4}+O\left(p^{5}\right)$ & $2 p+2 p^{2}+p^{3}+O\left(p^{4}\right)$ \\
\hline 5 & -2 & $3 p+3 p^{2}+3 p^{3}+O\left(p^{4}\right)$ & $2 p+2 p^{2}+4 p^{3}+O\left(p^{4}\right)$ \\
\hline 7 & -2 & $2 p+2 p^{2}+O\left(p^{3}\right)$ & $6 p+2 p^{2}+O\left(p^{3}\right)$ \\
\hline 11 & -6 & $10 p+O\left(p^{2}\right)$ & $2 p+O\left(p^{2}\right)$ \\
\hline 13 & 2 & $9 p+O\left(p^{2}\right)$ & $6 p+O\left(p^{2}\right)$ \\
\hline
\end{tabular}


$E=136 \mathrm{~b} 1, C_{\mathrm{Sym}^{2} E}=68^{2}, S_{1}=\{2\}, \xi_{\mathrm{Sym}^{2} E}=2$

\begin{tabular}{|c|c|c|c|}
\hline$p$ & $a_{p}(E)$ & $\mathbf{L}_{p}^{\mathrm{imp}}\left(\mathrm{Sym}^{2} E, 1\right)^{\prime}$ & $\mathcal{L}_{p}^{\mathrm{an}}\left(\mathrm{Sym}^{2} E\right)$ \\
\hline 3 & 2 & $2 p+2 p^{2}+2 p^{3}+2 p^{4}+O\left(p^{5}\right)$ & $2+2 p+2 p^{2}+2 p^{3}+O\left(p^{4}\right)$ \\
\hline 11 & 2 & $3 p+O\left(p^{2}\right)$ & $2 p+O\left(p^{2}\right)$ \\
\hline 13 & -6 & $p+O\left(p^{2}\right)$ & $2 p+O\left(p^{2}\right)$ \\
\hline
\end{tabular}

$E=140 \mathrm{a} 1, C_{\mathrm{Sym}^{2} E}=70^{2}, S_{1}=\{2\}, \xi_{\mathrm{Sym}^{2} E}=\frac{8}{3}$

\begin{tabular}{|c|c|c|c|}
\hline$p$ & $a_{p}(E)$ & $\mathbf{L}_{p}^{\text {imp }}\left(\mathrm{Sym}^{2} E, 1\right)^{\prime}$ & $\mathcal{L}_{p}^{\text {an }}\left(\mathrm{Sym}^{2} E\right)$ \\
\hline 3 & 1 & $2 p+p^{2}+O\left(p^{4}\right)$ & $p+2 p^{2}+2 p^{3}+O\left(p^{4}\right)$ \\
\hline 11 & 3 & $p+O\left(p^{2}\right)$ & $3 p+O\left(p^{2}\right)$ \\
\hline 13 & -1 & $O\left(p^{2}\right)$ & $O(p)$ \\
\hline
\end{tabular}

$E=140 \mathrm{~b} 1, C_{\mathrm{Sym}^{2} E}=70^{2}, S_{1}=\{2\}, \xi_{\mathrm{Sym}^{2} E}=\frac{8}{3}$

\begin{tabular}{|c|c|c|c|}
\hline$p$ & $a_{p}(E)$ & $\mathbf{L}_{p}^{\text {imp }}\left(\mathrm{Sym}^{2} E, 1\right)^{\prime}$ & $\mathcal{L}_{p}^{\text {an }}\left(\mathrm{Sym}^{2} E\right)$ \\
\hline 11 & -5 & $2 p+O\left(p^{2}\right)$ & $8 p+O\left(p^{2}\right)$ \\
\hline 13 & -3 & $8 p+O\left(p^{2}\right)$ & $5 p+O\left(p^{2}\right)$ \\
\hline
\end{tabular}

$E=148 \mathrm{a} 1, C_{\mathrm{Sym}^{2} E}=74^{2}, S_{1}=\{2\}, \xi_{\mathrm{Sym}^{2} E}=\frac{8}{3}$

\begin{tabular}{|c|c|c|c|}
\hline$p$ & $a_{p}(E)$ & $\mathbf{L}_{p}^{\text {imp }}\left(\mathrm{Sym}^{2} E, 1\right)^{\prime}$ & $\mathcal{L}_{p}^{\text {an }}\left(\mathrm{Sym}^{2} E\right)$ \\
\hline 3 & -1 & $2 p+p^{2}+O\left(p^{4}\right)$ & $p+2 p^{2}+2 p^{3}+O\left(p^{4}\right)$ \\
\hline 5 & -4 & $3 p+4 p^{2}+O\left(p^{3}\right)$ & $3+2 p+O\left(p^{2}\right)$ \\
\hline 7 & -3 & $p+3 p^{2}+O\left(p^{3}\right)$ & $6 p+4 p^{2}+O\left(p^{3}\right)$ \\
\hline 11 & 5 & $8 p+O\left(p^{2}\right)$ & $10 p+O\left(p^{2}\right)$ \\
\hline
\end{tabular}

$E=152 \mathrm{a} 1, C_{\mathrm{Sym}^{2} E}=76^{2}, S_{1}=\{2\}, \xi_{\mathrm{Sym}^{2} E}=2$

\begin{tabular}{|c|c|c|c|}
\hline$p$ & $a_{p}(E)$ & $\mathbf{L}_{p}^{\text {imp }}\left(\mathrm{Sym}^{2} E, 1\right)^{\prime}$ & $\mathcal{L}_{p}^{\text {an }}\left(\mathrm{Sym}^{2} E\right)$ \\
\hline 3 & -2 & $2 p+O\left(p^{5}\right)$ & $2+O\left(p^{4}\right)$ \\
\hline 5 & -1 & $p+2 p^{2}+O\left(p^{3}\right)$ & $1+4 p+O\left(p^{2}\right)$ \\
\hline 7 & -3 & $p+6 p^{2}+O\left(p^{3}\right)$ & $p+O\left(p^{3}\right)$ \\
\hline 11 & -3 & $3 p+O\left(p^{2}\right)$ & $p+O\left(p^{2}\right)$ \\
\hline 13 & -4 & $4 p+O\left(p^{2}\right)$ & $3 p+O\left(p^{2}\right)$ \\
\hline
\end{tabular}

$E=152 \mathrm{~b} 1, C_{\mathrm{Sym}^{2} E}=76^{2}, S_{1}=\{2\}, \xi_{\mathrm{Sym}^{2} E}=2$

\begin{tabular}{|c|c|c|c|}
\hline$p$ & $a_{p}(E)$ & $\mathbf{L}_{p}^{\text {imp }}\left(\mathrm{Sym}^{2} E, 1\right)^{\prime}$ & $\mathcal{L}_{p}^{\text {an }}\left(\mathrm{Sym}^{2} E\right)$ \\
\hline 3 & 1 & $2 p+2 p^{2}+2 p^{3}+2 p^{4}+O\left(p^{5}\right)$ & $1+2 p+O\left(p^{4}\right)$ \\
\hline 7 & 3 & $p+O\left(p^{3}\right)$ & $p+p^{2}+O\left(p^{3}\right)$ \\
\hline 11 & 2 & $8 p+O\left(p^{2}\right)$ & $9 p+O\left(p^{2}\right)$ \\
\hline 13 & 1 & $7 p+O\left(p^{2}\right)$ & $8+O(p)$ \\
\hline
\end{tabular}


$E=156 \mathrm{a} 1, C_{\mathrm{Sym}^{2} E}=78^{2}, S_{1}=\{2\}, \xi_{\mathrm{Sym}^{2} E}=\frac{8}{3}$

\begin{tabular}{|c|c|c|c|}
\hline$p$ & $a_{p}(E)$ & $\mathbf{L}_{p}^{\text {imp }}\left(\mathrm{Sym}^{2} E, 1\right)^{\prime}$ & $\mathcal{L}_{p}^{\text {an }}\left(\mathrm{Sym}^{2} E\right)$ \\
\hline 5 & -4 & $2 p+p^{2}+O\left(p^{3}\right)$ & $2+3 p+O\left(p^{2}\right)$ \\
\hline 7 & -2 & $4 p+O\left(p^{3}\right)$ & $2 p+2 p^{2}+O\left(p^{3}\right)$ \\
\hline 11 & -4 & $7 p+O\left(p^{2}\right)$ & $5 p+O\left(p^{2}\right)$ \\
\hline
\end{tabular}

$E=156 \mathrm{~b} 1, C_{\mathrm{Sym}^{2} E}=78^{2}, S_{1}=\{2\}, \xi_{\mathrm{Sym}^{2} E}=\frac{8}{3}$

\begin{tabular}{|c|c|c|c|}
\hline$p$ & $a_{p}(E)$ & $\mathbf{L}_{p}^{\mathrm{imp}}\left(\mathrm{Sym}^{2} E, 1\right)^{\prime}$ & $\mathcal{L}_{p}^{\mathrm{an}}\left(\mathrm{Sym}^{2} E\right)$ \\
\hline 7 & 2 & $6 p+O\left(p^{3}\right)$ & $3 p+3 p^{2}+O\left(p^{3}\right)$ \\
\hline
\end{tabular}

$E=160 \mathrm{a} 1, C_{\mathrm{Sym}^{2} E}=40^{2}, S_{1}=\{2\}, \xi_{\mathrm{Sym}^{2} E}=1$

\begin{tabular}{|c|c|c|c|}
\hline$p$ & $a_{p}(E)$ & $\mathbf{L}_{p}^{\text {imp }}\left(\mathrm{Sym}^{2} E, 1\right)^{\prime}$ & $\mathcal{L}_{p}^{\text {an }}\left(\mathrm{Sym}^{2} E\right)$ \\
\hline 3 & -2 & $2 p+2 p^{2}+p^{3}+p^{4}+O\left(p^{5}\right)$ & $1+2 p+O\left(p^{4}\right)$ \\
\hline 7 & -2 & $p+3 p^{2}+O\left(p^{3}\right)$ & $6 p+O\left(p^{3}\right)$ \\
\hline 11 & -4 & $2 p+O\left(p^{2}\right)$ & $8 p+O\left(p^{2}\right)$ \\
\hline 13 & -6 & $10 p+O\left(p^{2}\right)$ & $p+O\left(p^{2}\right)$ \\
\hline
\end{tabular}

$E=168 \mathrm{a} 1, C_{\mathrm{Sym}^{2} E}=84^{2}, S_{1}=\{2\}, \xi_{\mathrm{Sym}^{2} E}=2$

\begin{tabular}{|c|c|c|c|}
\hline$p$ & $a_{p}(E)$ & $\mathbf{L}_{p}^{\text {imp }}\left(\mathrm{Sym}^{2} E, 1\right)^{\prime}$ & $\mathcal{L}_{p}^{\text {an }}\left(\mathrm{Sym}^{2} E\right)$ \\
\hline 5 & 2 & $p+p^{2}+3 p^{3}+O\left(p^{4}\right)$ & $4 p+p^{3}+O\left(p^{4}\right)$ \\
\hline 13 & -2 & $3 p+O\left(p^{2}\right)$ & $2 p+O\left(p^{2}\right)$ \\
\hline
\end{tabular}

$E=168 \mathrm{~b} 1, C_{\mathrm{Sym}^{2} E}=84^{2}, S_{1}=\{2\}, \xi_{\mathrm{Sym}^{2} E}=2$

\begin{tabular}{|c|c|c|c|}
\hline$p$ & $a_{p}(E)$ & $\mathbf{L}_{p}^{\text {imp }}\left(\mathrm{Sym}^{2} E, 1\right)^{\prime}$ & $\mathcal{L}_{p}^{\text {an }}\left(\mathrm{Sym}^{2} E\right)$ \\
\hline 5 & 2 & $4 p^{2}+2 p^{3}+O\left(p^{4}\right)$ & $p^{2}+O\left(p^{4}\right)$ \\
\hline 13 & 6 & $10 p+O\left(p^{2}\right)$ & $7 p+O\left(p^{2}\right)$ \\
\hline
\end{tabular}

$E=172 \mathrm{a} 1, C_{\mathrm{Sym}^{2} E}=86^{2}, S_{1}=\{2\}, \xi_{\mathrm{Sym}^{2} E}=\frac{8}{3}$

\begin{tabular}{|c|c|c|c|}
\hline$p$ & $a_{p}(E)$ & $\mathbf{L}_{p}^{\text {imp }}\left(\mathrm{Sym}^{2} E, 1\right)^{\prime}$ & $\mathcal{L}_{p}^{\text {an }}\left(\mathrm{Sym}^{2} E\right)$ \\
\hline 3 & -2 & $2+p+O\left(p^{4}\right)$ & $2+2 p+2 p^{3}+O\left(p^{4}\right)$ \\
\hline 7 & -4 & $4 p+2 p^{2}+O\left(p^{3}\right)$ & $3 p+3 p^{2}+O\left(p^{3}\right)$ \\
\hline 11 & -3 & $3 p+O\left(p^{2}\right)$ & $9 p+O\left(p^{2}\right)$ \\
\hline 13 & -1 & $9 p+O\left(p^{2}\right)$ & $4+O(p)$ \\
\hline
\end{tabular}

$E=184 \mathrm{a} 1, C_{\mathrm{Sym}^{2} E}=92^{2}, S_{1}=\{2\}, \xi_{\mathrm{Sym}^{2} E}=2$

\begin{tabular}{|c|c|c|c|}
\hline$p$ & $a_{p}(E)$ & $\mathbf{L}_{p}^{\text {imp }}\left(\mathrm{Sym}^{2} E, 1\right)^{\prime}$ & $\mathcal{L}_{p}^{\text {an }}\left(\mathrm{Sym}^{2} E\right)$ \\
\hline 3 & -1 & $2 p^{2}+p^{3}+p^{4}+O\left(p^{5}\right)$ & $p+p^{3}+O\left(p^{4}\right)$ \\
\hline 5 & -4 & $2 p^{2}+O\left(p^{3}\right)$ & $p+O\left(p^{2}\right)$ \\
\hline 7 & 2 & $p+6 p^{2}+O\left(p^{3}\right)$ & $3 p+2 p^{2}+O\left(p^{3}\right)$ \\
\hline 11 & -4 & $6 p+O\left(p^{2}\right)$ & $p+O\left(p^{2}\right)$ \\
\hline 13 & -5 & $3 p+O\left(p^{2}\right)$ & $4 p+O\left(p^{2}\right)$ \\
\hline
\end{tabular}


$E=184 \mathrm{~b} 1, C_{\mathrm{Sym}^{2} E}=92^{2}, S_{1}=\{2\}, \xi_{\mathrm{Sym}^{2} E}=2$

\begin{tabular}{|c|c|c|c|}
\hline$p$ & $a_{p}(E)$ & $\mathbf{L}_{p}^{\text {imp }}\left(\mathrm{Sym}^{2} E, 1\right)^{\prime}$ & $\mathcal{L}_{p}^{\text {an }}\left(\mathrm{Sym}^{2} E\right)$ \\
\hline 3 & -1 & $2 p^{2}+2 p^{3}+O\left(p^{5}\right)$ & $p+2 p^{2}+2 p^{3}+O\left(p^{4}\right)$ \\
\hline 5 & -2 & $p+3 p^{2}+O\left(p^{4}\right)$ & $4 p+3 p^{2}+2 p^{3}+O\left(p^{4}\right)$ \\
\hline 7 & -4 & $3 p+p^{2}+O\left(p^{3}\right)$ & $3 p+6 p^{2}+O\left(p^{3}\right)$ \\
\hline 11 & -2 & $4 p+O\left(p^{2}\right)$ & $10 p+O\left(p^{2}\right)$ \\
\hline 13 & 7 & $3 p+O\left(p^{2}\right)$ & $6 p+O\left(p^{2}\right)$ \\
\hline
\end{tabular}

$E=184 \mathrm{c} 1, C_{\mathrm{Sym}^{2} E}=92^{2}, S_{1}=\{2\}, \xi_{\mathrm{Sym}^{2} E}=2$

\begin{tabular}{|c|c|c|c|}
\hline$p$ & $a_{p}(E)$ & $\mathbf{L}_{p}^{\text {imp }}\left(\mathrm{Sym}^{2} E, 1\right)^{\prime}$ & $\mathcal{L}_{p}^{\text {an }}\left(\mathrm{Sym}^{2} E\right)$ \\
\hline 7 & 4 & $5 p+5 p^{2}+O\left(p^{3}\right)$ & $5 p+4 p^{2}+O\left(p^{3}\right)$ \\
\hline 11 & 6 & $8 p+O\left(p^{2}\right)$ & $6 p+O\left(p^{2}\right)$ \\
\hline 13 & -2 & $7 p+O\left(p^{2}\right)$ & $9 p+O\left(p^{2}\right)$ \\
\hline
\end{tabular}

$E=184 \mathrm{~d} 1, C_{\mathrm{Sym}^{2} E}=92^{2}, S_{1}=\{2\}, \xi_{\mathrm{Sym}^{2} E}=2$

\begin{tabular}{|c|c|c|c|}
\hline$p$ & $a_{p}(E)$ & $\mathbf{L}_{p}^{\text {imp }}\left(\mathrm{Sym}^{2} E, 1\right)^{\prime}$ & $\mathcal{L}_{p}^{\text {an }}\left(\mathrm{Sym}^{2} E\right)$ \\
\hline 7 & -2 & $p+O\left(p^{3}\right)$ & $3 p+5 p^{2}+O\left(p^{3}\right)$ \\
\hline 13 & -5 & $p+O\left(p^{2}\right)$ & $10 p+O\left(p^{2}\right)$ \\
\hline
\end{tabular}

$E=200 \mathrm{a} 1, C_{\mathrm{Sym}^{2} E}=20^{2}, S_{1}=\{2,5\}, \xi_{\mathrm{Sym}^{2} E}=\frac{2}{3}$

\begin{tabular}{|c|c|c|c|}
\hline$p$ & $a_{p}(E)$ & $\mathbf{L}_{p}^{\mathrm{imp}}\left(\mathrm{Sym}^{2} E, 1\right)^{\prime}$ & $\mathcal{L}_{p}^{\mathrm{an}}\left(\mathrm{Sym}^{2} E\right)$ \\
\hline 7 & 2 & $5 p+6 p^{2}+O\left(p^{3}\right)$ & $3 p+2 p^{2}+O\left(p^{3}\right)$ \\
\hline 11 & 1 & $O\left(p^{2}\right)$ & $O(p)$ \\
\hline 13 & 4 & $6 p+O\left(p^{2}\right)$ & $7 p+O\left(p^{2}\right)$ \\
\hline
\end{tabular}

$E=204 \mathrm{a} 1, C_{\mathrm{Sym}^{2} E}=102^{2}, S_{1}=\{2\}, \xi_{\mathrm{Sym}^{2} E}=\frac{8}{3}$

\begin{tabular}{|c|c|c|c|}
\hline$p$ & $a_{p}(E)$ & $\mathbf{L}_{p}^{\text {imp }}\left(\mathrm{Sym}^{2} E, 1\right)^{\prime}$ & $\mathcal{L}_{p}^{\text {an }}\left(\mathrm{Sym}^{2} E\right)$ \\
\hline 5 & -1 & $2 p+p^{2}+O\left(p^{3}\right)$ & $4+2 p+O\left(p^{2}\right)$ \\
\hline 7 & 4 & $6 p^{3}+O\left(p^{4}\right)$ & $p^{3}+O\left(p^{4}\right)$ \\
\hline 11 & 3 & $8 p+O\left(p^{2}\right)$ & $2 p+O\left(p^{2}\right)$ \\
\hline 13 & 3 & $3 p+O\left(p^{2}\right)$ & $10 p+O\left(p^{2}\right)$ \\
\hline
\end{tabular}

$E=204 \mathrm{~b} 1, C_{\mathrm{Sym}^{2} E}=102^{2}, S_{1}=\{2\}, \xi_{\mathrm{Sym}^{2} E}=\frac{8}{3}$

\begin{tabular}{|c|c|c|c|}
\hline$p$ & $a_{p}(E)$ & $\mathbf{L}_{p}^{\text {imp }}\left(\mathrm{Sym}^{2} E, 1\right)^{\prime}$ & $\mathcal{L}_{p}^{\text {an }}\left(\mathrm{Sym}^{2} E\right)$ \\
\hline 5 & 1 & $2 p^{2}+O\left(p^{3}\right)$ & $4 p+O\left(p^{2}\right)$ \\
\hline 11 & 5 & $10 p+O\left(p^{2}\right)$ & $7 p+O\left(p^{2}\right)$ \\
\hline 13 & -5 & $8 p+O\left(p^{2}\right)$ & $8 p+O\left(p^{2}\right)$ \\
\hline
\end{tabular}


$E=208 \mathrm{a} 1, C_{\mathrm{Sym}^{2} E}=26^{2}, S_{1}=\{\}, \xi_{\mathrm{Sym}^{2} E}=\frac{1}{2}$

\begin{tabular}{|c|c|c|c|}
\hline$p$ & $a_{p}(E)$ & $\mathbf{L}_{p}^{\mathrm{imp}}\left(\mathrm{Sym}^{2} E, 1\right)^{\prime}$ & $\mathcal{L}_{p}^{\mathrm{an}}\left(\mathrm{Sym}^{2} E\right)$ \\
\hline 3 & -1 & $p+2 p^{2}+2 p^{4}+O\left(p^{5}\right)$ & $2+2 p^{2}+2 p^{3}+O\left(p^{4}\right)$ \\
\hline 5 & -3 & $2 p+3 p^{2}+4 p^{3}+O\left(p^{4}\right)$ & $2 p+p^{2}+2 p^{3}+O\left(p^{4}\right)$ \\
\hline 7 & 1 & $6 p+O\left(p^{2}\right)$ & $1+O(p)$ \\
\hline 11 & -6 & $7 p+O\left(p^{2}\right)$ & $10 p+O\left(p^{2}\right)$ \\
\hline
\end{tabular}

$E=208 \mathrm{~d} 1, C_{\mathrm{Sym}^{2} E}=26^{2}, S_{1}=\{\}, \xi_{\mathrm{Sym}^{2} E}=\frac{1}{2}$

\begin{tabular}{|c|c|c|c|}
\hline$p$ & $a_{p}(E)$ & $\mathbf{L}_{p}^{\text {imp }}\left(\mathrm{Sym}^{2} E, 1\right)^{\prime}$ & $\mathcal{L}_{p}^{\text {an }}\left(\mathrm{Sym}^{2} E\right)$ \\
\hline 5 & -1 & $4 p+4 p^{2}+O\left(p^{3}\right)$ & $1+p+O\left(p^{2}\right)$ \\
\hline 7 & -1 & $3 p^{2}+O\left(p^{3}\right)$ & $4 p+O\left(p^{2}\right)$ \\
\hline 11 & 2 & $6 p+O\left(p^{2}\right)$ & $5 p+O\left(p^{2}\right)$ \\
\hline
\end{tabular}

$E=212 \mathrm{a} 1, C_{\mathrm{Sym}^{2} E}=106^{2}, S_{1}=\{2\}, \xi_{\mathrm{Sym}^{2} E}=\frac{8}{3}$

\begin{tabular}{|c|c|c|c|}
\hline$p$ & $a_{p}(E)$ & $\mathbf{L}_{p}^{\text {imp }}\left(\mathrm{Sym}^{2} E, 1\right)^{\prime}$ & $\mathcal{L}_{p}^{\text {an }}\left(\mathrm{Sym}^{2} E\right)$ \\
\hline 3 & -1 & $2+2 p+p^{3}+O\left(p^{4}\right)$ & $1+p+p^{2}+p^{3}+O\left(p^{4}\right)$ \\
\hline 5 & -2 & $p^{2}+2 p^{3}+O\left(p^{4}\right)$ & $3 p^{2}+3 p^{3}+O\left(p^{4}\right)$ \\
\hline 7 & -2 & $3 p+4 p^{2}+O\left(p^{3}\right)$ & $5 p+3 p^{2}+O\left(p^{3}\right)$ \\
\hline 11 & 2 & $8 p+O\left(p^{2}\right)$ & $4 p+O\left(p^{2}\right)$ \\
\hline 13 & -7 & $11 p+O\left(p^{2}\right)$ & $10 p+O\left(p^{2}\right)$ \\
\hline
\end{tabular}

$E=212 \mathrm{~b} 1, C_{\mathrm{Sym}^{2} E}=106^{2}, S_{1}=\{2\}, \xi_{\mathrm{Sym}^{2} E}=\frac{8}{3}$

\begin{tabular}{|c|c|c|c|}
\hline$p$ & $a_{p}(E)$ & $\mathbf{L}_{p}^{\text {imp }}\left(\mathrm{Sym}^{2} E, 1\right)^{\prime}$ & $\mathcal{L}_{p}^{\text {an }}\left(\mathrm{Sym}^{2} E\right)$ \\
\hline 3 & 2 & $1+p+p^{2}+2 p^{3}+O\left(p^{4}\right)$ & $1+p^{2}+p^{3}+O\left(p^{4}\right)$ \\
\hline 5 & 2 & $4 p+p^{2}+3 p^{3}+O\left(p^{4}\right)$ & $2 p+3 p^{2}+3 p^{3}+O\left(p^{4}\right)$ \\
\hline 11 & -4 & $10 p+O\left(p^{2}\right)$ & $4 p+O\left(p^{2}\right)$ \\
\hline 13 & -2 & $3 p+O\left(p^{2}\right)$ & $8 p+O\left(p^{2}\right)$ \\
\hline
\end{tabular}

$E=216 \mathrm{a} 1, C_{\mathrm{Sym}^{2} E}=36^{2}, S_{1}=\{2,3\}, \xi_{\mathrm{Sym}^{2} E}=\frac{2}{3}$

\begin{tabular}{|c|c|c|c|}
\hline$p$ & $a_{p}(E)$ & $\mathbf{L}_{p}^{\text {imp }}\left(\mathrm{Sym}^{2} E, 1\right)^{\prime}$ & $\mathcal{L}_{p}^{\text {an }}\left(\mathrm{Sym}^{2} E\right)$ \\
\hline 5 & -4 & $2 p+3 p^{2}+O\left(p^{3}\right)$ & $3+p+O\left(p^{2}\right)$ \\
\hline 7 & -3 & $p+4 p^{2}+O\left(p^{3}\right)$ & $3 p+p^{2}+O\left(p^{3}\right)$ \\
\hline 11 & -4 & $6 p+O\left(p^{2}\right)$ & $3 p+O\left(p^{2}\right)$ \\
\hline 13 & 1 & $4 p+O\left(p^{2}\right)$ & $10+O(p)$ \\
\hline
\end{tabular}

$E=216 \mathrm{c} 1, C_{\mathrm{Sym}^{2} E}=36^{2}, S_{1}=\{2,3\}, \xi_{\mathrm{Sym}^{2} E}=\frac{2}{3}$

\begin{tabular}{|c|c|c|c|}
\hline$p$ & $a_{p}(E)$ & $\mathbf{L}_{p}^{\text {imp }}\left(\mathrm{Sym}^{2} E, 1\right)^{\prime}$ & $\mathcal{L}_{p}^{\text {an }}\left(\mathrm{Sym}^{2} E\right)$ \\
\hline 5 & 1 & $2 p+O\left(p^{2}\right)$ & $1+O(p)$ \\
\hline 7 & 3 & $4 p+2 p^{2}+O\left(p^{3}\right)$ & $5 p+5 p^{2}+O\left(p^{3}\right)$ \\
\hline 11 & -5 & $10 p+O\left(p^{2}\right)$ & $6 p+O\left(p^{2}\right)$ \\
\hline 13 & 4 & $2 p+O\left(p^{2}\right)$ & $11 p+O\left(p^{2}\right)$ \\
\hline
\end{tabular}




$E=220 \mathrm{a} 1, C_{\mathrm{Sym}^{2} E}=110^{2}, S_{1}=\{2\}, \xi_{\mathrm{Sym}^{2} E}=\frac{8}{3}$
\begin{tabular}{|c|c|c|c|}
\hline$p$ & $a_{p}(E)$ & $\mathbf{L}_{p}^{\text {imp }}\left(\mathrm{Sym}^{2} E, 1\right)^{\prime}$ & $\mathcal{L}_{p}^{\text {an }}\left(\mathrm{Sym}^{2} E\right)$ \\
\hline 3 & -2 & $2+O\left(p^{2}\right)$ & $2+p+O\left(p^{2}\right)$ \\
\hline 7 & -4 & $p+O\left(p^{3}\right)$ & $6 p+4 p^{2}+O\left(p^{3}\right)$ \\
\hline 13 & -4 & $2 p+O\left(p^{2}\right)$ & $6 p+O\left(p^{2}\right)$ \\
\hline
\end{tabular}

$E=220 \mathrm{~b} 1, C_{\mathrm{Sym}^{2} E}=110^{2}, S_{1}=\{2\}, \xi_{\mathrm{Sym}^{2} E}=\frac{8}{3}$

\begin{tabular}{|c|c|c|c|}
\hline$p$ & $a_{p}(E)$ & $\mathbf{L}_{p}^{\text {imp }}\left(\mathrm{Sym}^{2} E, 1\right)^{\prime}$ & $\mathcal{L}_{p}^{\text {an }}\left(\mathrm{Sym}^{2} E\right)$ \\
\hline 3 & 2 & $1+p^{2}+O\left(p^{3}\right)$ & $1+2 p+p^{2}+O\left(p^{3}\right)$ \\
\hline
\end{tabular}

$E=224 \mathrm{a} 1, C_{\mathrm{Sym}^{2} E}=56^{2}, S_{1}=\{2\}, \xi_{\mathrm{Sym}^{2} E}=1$

\begin{tabular}{|c|c|c|c|}
\hline$p$ & $a_{p}(E)$ & $\mathbf{L}_{p}^{\text {imp }}\left(\mathrm{Sym}^{2} E, 1\right)^{\prime}$ & $\mathcal{L}_{p}^{\text {an }}\left(\mathrm{Sym}^{2} E\right)$ \\
\hline 3 & -2 & $p^{3}+O\left(p^{4}\right)$ & $2 p^{2}+O\left(p^{3}\right)$ \\
\hline 11 & -4 & $10 p+O\left(p^{2}\right)$ & $7 p+O\left(p^{2}\right)$ \\
\hline 13 & -4 & $8 p+O\left(p^{2}\right)$ & $12 p+O\left(p^{2}\right)$ \\
\hline
\end{tabular}

$E=228 \mathrm{a} 1, C_{\mathrm{Sym}^{2} E}=114^{2}, S_{1}=\{2\}, \xi_{\mathrm{Sym}^{2} E}=\frac{8}{3}$

\begin{tabular}{|c|c|c|c|}
\hline$p$ & $a_{p}(E)$ & $\mathbf{L}_{p}^{\text {imp }}\left(\mathrm{Sym}^{2} E, 1\right)^{\prime}$ & $\mathcal{L}_{p}^{\text {an }}\left(\mathrm{Sym}^{2} E\right)$ \\
\hline 5 & 2 & $p+O\left(p^{3}\right)$ & $3 p+2 p^{2}+O\left(p^{3}\right)$ \\
\hline 11 & 2 & $9 p+O\left(p^{2}\right)$ & $10 p+O\left(p^{2}\right)$ \\
\hline 13 & 2 & $11 p+O\left(p^{2}\right)$ & $12 p+O\left(p^{2}\right)$ \\
\hline
\end{tabular}

$E=228 \mathrm{~b} 1, C_{\mathrm{Sym}^{2} E}=114^{2}, S_{1}=\{2\}, \xi_{\mathrm{Sym}^{2} E}=\frac{8}{3}$

\begin{tabular}{|c|c|c|c|}
\hline$p$ & $a_{p}(E)$ & $\mathbf{L}_{p}^{\text {imp }}\left(\mathrm{Sym}^{2} E, 1\right)^{\prime}$ & $\mathcal{L}_{p}^{\text {an }}\left(\mathrm{Sym}^{2} E\right)$ \\
\hline 5 & -3 & $2 p+4 p^{2}+O\left(p^{3}\right)$ & $p+4 p^{2}+O\left(p^{3}\right)$ \\
\hline 7 & 1 & $6 p+O\left(p^{2}\right)$ & $5+O(p)$ \\
\hline 11 & -5 & $2 p+O\left(p^{2}\right)$ & $8 p+O\left(p^{2}\right)$ \\
\hline 13 & -6 & $p+O\left(p^{2}\right)$ & $8 p+O\left(p^{2}\right)$ \\
\hline
\end{tabular}

$E=232 \mathrm{a} 1, C_{\mathrm{Sym}^{2} E}=116^{2}, S_{1}=\{2\}, \xi_{\mathrm{Sym}^{2} E}=2$

\begin{tabular}{|c|c|c|c|}
\hline$p$ & $a_{p}(E)$ & $\mathbf{L}_{p}^{\text {imp }}\left(\mathrm{Sym}^{2} E, 1\right)^{\prime}$ & $\mathcal{L}_{p}^{\text {an }}\left(\mathrm{Sym}^{2} E\right)$ \\
\hline 3 & -1 & $p+O\left(p^{4}\right)$ & $2+2 p^{2}+O\left(p^{3}\right)$ \\
\hline 5 & -3 & $p+3 p^{2}+O\left(p^{3}\right)$ & $4 p+p^{2}+O\left(p^{3}\right)$ \\
\hline 7 & 2 & $p+O\left(p^{3}\right)$ & $3 p+5 p^{2}+O\left(p^{3}\right)$ \\
\hline 11 & -3 & $3 p+O\left(p^{2}\right)$ & $p+O\left(p^{2}\right)$ \\
\hline 13 & -5 & $2 p+O\left(p^{2}\right)$ & $7 p+O\left(p^{2}\right)$ \\
\hline
\end{tabular}


$E=232 \mathrm{~b} 1, C_{\mathrm{Sym}^{2} E}=116^{2}, S_{1}=\{2\}, \xi_{\mathrm{Sym}^{2} E}=2$

\begin{tabular}{|c|c|c|c|}
\hline$p$ & $a_{p}(E)$ & $\mathbf{L}_{p}^{\text {imp }}\left(\mathrm{Sym}^{2} E, 1\right)^{\prime}$ & $\mathcal{L}_{p}^{\mathrm{an}}\left(\mathrm{Sym}^{2} E\right)$ \\
\hline 3 & 1 & $p+2 p^{3}+O\left(p^{4}\right)$ & $2+O\left(p^{3}\right)$ \\
\hline 5 & 1 & $3 p+O\left(p^{2}\right)$ & $3+O(p)$ \\
\hline 7 & 2 & $2 p+O\left(p^{3}\right)$ & $6 p+3 p^{2}+O\left(p^{3}\right)$ \\
\hline 11 & 3 & $6 p+O\left(p^{2}\right)$ & $2 p+O\left(p^{2}\right)$ \\
\hline 13 & -1 & $O(p)$ & $O(1)$ \\
\hline
\end{tabular}

$E=236 \mathrm{a} 1, C_{\mathrm{Sym}^{2} E}=118^{2}, S_{1}=\{2\}, \xi_{\mathrm{Sym}^{2} E}=\frac{8}{3}$

\begin{tabular}{|c|c|c|c|}
\hline$p$ & $a_{p}(E)$ & $\mathbf{L}_{p}^{\text {imp }}\left(\mathrm{Sym}^{2} E, 1\right)^{\prime}$ & $\mathcal{L}_{p}^{\text {an }}\left(\mathrm{Sym}^{2} E\right)$ \\
\hline 3 & -1 & $2 p^{2}+O\left(p^{3}\right)$ & $p^{2}+O\left(p^{3}\right)$ \\
\hline 5 & -1 & $4 p+O\left(p^{2}\right)$ & $3+O(p)$ \\
\hline 7 & -3 & $6 p+O\left(p^{3}\right)$ & $p+5 p^{2}+O\left(p^{3}\right)$ \\
\hline 11 & -2 & $3 p+O\left(p^{2}\right)$ & $7 p+O\left(p^{2}\right)$ \\
\hline
\end{tabular}

$E=236 \mathrm{~b} 1, C_{\mathrm{Sym}^{2} E}=118^{2}, S_{1}=\{2\}, \xi_{\mathrm{Sym}^{2} E}=\frac{8}{3}$

\begin{tabular}{|c|c|c|c|}
\hline$p$ & $a_{p}(E)$ & $\mathbf{L}_{p}^{\text {imp }}\left(\mathrm{Sym}^{2} E, 1\right)^{\prime}$ & $\mathcal{L}_{p}^{\text {an }}\left(\mathrm{Sym}^{2} E\right)$ \\
\hline 3 & 1 & $2 p^{2}+O\left(p^{4}\right)$ & $p^{2}+O\left(p^{4}\right)$ \\
\hline 5 & 3 & $2 p^{3}+O\left(p^{4}\right)$ & $p^{3}+O\left(p^{4}\right)$ \\
\hline 7 & -1 & $6+O(p)$ & $5 p^{-1}+O(1)$ \\
\hline 11 & 6 & $5 p+O\left(p^{2}\right)$ & $9 p+O\left(p^{2}\right)$ \\
\hline 13 & -4 & $7 p+O\left(p^{2}\right)$ & $8 p+O\left(p^{2}\right)$ \\
\hline
\end{tabular}

$E=240 \mathrm{~b} 1, C_{\mathrm{Sym}^{2} E}=30^{2}, S_{1}=\{\}, \xi_{\mathrm{Sym}^{2} E}=\frac{1}{2}$

\begin{tabular}{|c|c|c|c|}
\hline$p$ & $a_{p}(E)$ & $\mathbf{L}_{p}^{\text {imp }}\left(\mathrm{Sym}^{2} E, 1\right)^{\prime}$ & $\mathcal{L}_{p}^{\text {an }}\left(\mathrm{Sym}^{2} E\right)$ \\
\hline 7 & 4 & $p+2 p^{2}+O\left(p^{3}\right)$ & $4 p+3 p^{2}+O\left(p^{3}\right)$ \\
\hline 13 & 2 & $10 p+O\left(p^{2}\right)$ & $5 p+O\left(p^{2}\right)$ \\
\hline
\end{tabular}

$E=244 \mathrm{a} 1, C_{\mathrm{Sym}^{2} E}=122^{2}, S_{1}=\{2\}, \xi_{\mathrm{Sym}^{2} E}=\frac{8}{3}$

\begin{tabular}{|c|c|c|c|}
\hline$p$ & $a_{p}(E)$ & $\mathbf{L}_{p}^{\mathrm{imp}}\left(\mathrm{Sym}^{2} E, 1\right)^{\prime}$ & $\mathcal{L}_{p}^{\text {an }}\left(\mathrm{Sym}^{2} E\right)$ \\
\hline 5 & -3 & $p+4 p^{2}+O\left(p^{3}\right)$ & $3 p+O\left(p^{3}\right)$ \\
\hline 7 & -3 & $3 p+2 p^{2}+O\left(p^{3}\right)$ & $4 p+O\left(p^{3}\right)$ \\
\hline 11 & -1 & $4 p+O\left(p^{2}\right)$ & $2+O(p)$ \\
\hline 13 & 1 & $O\left(p^{2}\right)$ & $O(p)$ \\
\hline
\end{tabular}

$E=248 \mathrm{a} 1, C_{\mathrm{Sym}^{2} E}=124^{2}, S_{1}=\{2\}, \xi_{\mathrm{Sym}^{2} E}=2$

\begin{tabular}{|c|c|c|c|}
\hline$p$ & $a_{p}(E)$ & $\mathbf{L}_{p}^{\text {imp }}\left(\mathrm{Sym}^{2} E, 1\right)^{\prime}$ & $\mathcal{L}_{p}^{\text {an }}\left(\mathrm{Sym}^{2} E\right)$ \\
\hline 3 & -2 & $p^{2}+O\left(p^{4}\right)$ & $p+O\left(p^{3}\right)$ \\
\hline 5 & 1 & $3 p+O\left(p^{2}\right)$ & $3+O(p)$ \\
\hline 7 & -3 & $2 p+2 p^{2}+O\left(p^{3}\right)$ & $2 p+4 p^{2}+O\left(p^{3}\right)$ \\
\hline 11 & -2 & $7 p^{2}+O\left(p^{3}\right)$ & $p^{2}+O\left(p^{3}\right)$ \\
\hline 13 & -2 & $6 p+O\left(p^{2}\right)$ & $4 p+O\left(p^{2}\right)$ \\
\hline
\end{tabular}


$E=248 \mathrm{~b} 1, C_{\mathrm{Sym}^{2} E}=124^{2}, S_{1}=\{2\}, \xi_{\mathrm{Sym}^{2} E}=2$

\begin{tabular}{|c|c|c|c|}
\hline$p$ & $a_{p}(E)$ & $\mathbf{L}_{p}^{\mathrm{imp}}\left(\mathrm{Sym}^{2} E, 1\right)^{\prime}$ & $\mathcal{L}_{p}^{\mathrm{an}}\left(\mathrm{Sym}^{2} E\right)$ \\
\hline 3 & -2 & $2 p^{3}+O\left(p^{4}\right)$ & $2 p^{2}+O\left(p^{3}\right)$ \\
\hline 5 & 2 & $4 p+O\left(p^{3}\right)$ & $p+2 p^{2}+O\left(p^{3}\right)$ \\
\hline 11 & 2 & $5 p+5 p^{2}+O\left(p^{3}\right)$ & $7 p+5 p^{2}+O\left(p^{3}\right)$ \\
\hline 13 & 4 & $12 p+O\left(p^{2}\right)$ & $9 p+O\left(p^{2}\right)$ \\
\hline
\end{tabular}

$E=248 \mathrm{c} 1, C_{\mathrm{Sym}^{2} E}=124^{2}, S_{1}=\{2\}, \xi_{\mathrm{Sym}^{2} E}=2$

\begin{tabular}{|c|c|c|c|}
\hline$p$ & $a_{p}(E)$ & $\mathbf{L}_{p}^{\text {imp }}\left(\mathrm{Sym}^{2} E, 1\right)^{\prime}$ & $\mathcal{L}_{p}^{\text {an }}\left(\mathrm{Sym}^{2} E\right)$ \\
\hline 5 & -3 & $p^{3}+O\left(p^{4}\right)$ & $4 p^{3}+O\left(p^{4}\right)$ \\
\hline 7 & -3 & $6 p+O\left(p^{3}\right)$ & $6 p+6 p^{2}+O\left(p^{3}\right)$ \\
\hline 11 & 2 & $3 p^{2}+O\left(p^{3}\right)$ & $2 p^{2}+O\left(p^{3}\right)$ \\
\hline 13 & -4 & $7 p+O\left(p^{2}\right)$ & $2 p+O\left(p^{2}\right)$ \\
\hline
\end{tabular}

$E=256 \mathrm{a} 1, C_{\mathrm{Sym}^{2} E}=8^{2}, S_{1}=\{2\}, \xi_{\mathrm{Sym}^{2} E}=\frac{1}{6}$

\begin{tabular}{|c|c|c|c|}
\hline$p$ & $a_{p}(E)$ & $\mathbf{L}_{p}^{\text {imp }}\left(\mathrm{Sym}^{2} E, 1\right)^{\prime}$ & $\mathcal{L}_{p}^{\text {an }}\left(\mathrm{Sym}^{2} E\right)$ \\
\hline 3 & -2 & $p+p^{3}+O\left(p^{4}\right)$ & $p+p^{2}+p^{3}+O\left(p^{4}\right)$ \\
\hline 11 & -6 & $2 p+O\left(p^{2}\right)$ & $7 p+O\left(p^{2}\right)$ \\
\hline
\end{tabular}

$E=256 \mathrm{~b} 1, C_{\mathrm{Sym}^{2} E}=8^{2}, S_{1}=\{2\}, \xi_{\mathrm{Sym}^{2} E}=\frac{1}{6}$

\begin{tabular}{|c|c|c|c|}
\hline$p$ & $a_{p}(E)$ & $\mathbf{L}_{p}^{\text {imp }}\left(\mathrm{Sym}^{2} E, 1\right)^{\prime}$ & $\mathcal{L}_{p}^{\text {an }}\left(\mathrm{Sym}^{2} E\right)$ \\
\hline 5 & -4 & $p^{2}+O\left(p^{3}\right)$ & $p+O\left(p^{2}\right)$ \\
\hline 13 & -4 & $5 p+O\left(p^{2}\right)$ & $6 p+O\left(p^{2}\right)$ \\
\hline
\end{tabular}

$E=260 \mathrm{a} 1, C_{\mathrm{Sym}^{2} E}=130^{2}, S_{1}=\{2\}, \xi_{\mathrm{Sym}^{2} E}=\frac{8}{3}$

\begin{tabular}{|c|c|c|c|}
\hline$p$ & $a_{p}(E)$ & $\mathbf{L}_{p}^{\text {imp }}\left(\mathrm{Sym}^{2} E, 1\right)^{\prime}$ & $\mathcal{L}_{p}^{\text {an }}\left(\mathrm{Sym}^{2} E\right)$ \\
\hline 3 & 2 & $2+p^{2}+O\left(p^{3}\right)$ & $2+p+2 p^{2}+O\left(p^{3}\right)$ \\
\hline 7 & 2 & $p^{2}+O\left(p^{3}\right)$ & $4 p^{2}+O\left(p^{3}\right)$ \\
\hline 11 & 4 & $4 p+O\left(p^{2}\right)$ & $6 p+O\left(p^{2}\right)$ \\
\hline
\end{tabular}

$E=264 \mathrm{a} 1, C_{\mathrm{Sym}^{2} E}=132^{2}, S_{1}=\{2\}, \xi_{\mathrm{Sym}^{2} E}=2$

\begin{tabular}{|c|c|c|c|}
\hline$p$ & $a_{p}(E)$ & $\mathbf{L}_{p}^{\text {imp }}\left(\mathrm{Sym}^{2} E, 1\right)^{\prime}$ & $\mathcal{L}_{p}^{\text {an }}\left(\mathrm{Sym}^{2} E\right)$ \\
\hline 7 & 2 & $3 p+p^{2}+O\left(p^{3}\right)$ & $2 p+5 p^{2}+O\left(p^{3}\right)$ \\
\hline
\end{tabular}

$E=264 \mathrm{~b} 1, C_{\mathrm{Sym}^{2} E}=132^{2}, S_{1}=\{2\}, \xi_{\mathrm{Sym}^{2} E}=2$

\begin{tabular}{|c|c|c|c|}
\hline$p$ & $a_{p}(E)$ & $\mathbf{L}_{p}^{\text {imp }}\left(\mathrm{Sym}^{2} E, 1\right)^{\prime}$ & $\mathcal{L}_{p}^{\mathrm{an}}\left(\mathrm{Sym}^{2} E\right)$ \\
\hline 5 & 2 & $p+2 p^{2}+O\left(p^{3}\right)$ & $4 p+4 p^{2}+O\left(p^{3}\right)$ \\
\hline 13 & 2 & $12 p+O\left(p^{2}\right)$ & $8 p+O\left(p^{2}\right)$ \\
\hline
\end{tabular}


$E=264 \mathrm{c} 1, C_{\mathrm{Sym}^{2} E}=132^{2}, S_{1}=\{2\}, \xi_{\mathrm{Sym}^{2} E}=2$

\begin{tabular}{|c|c|c|c|}
\hline$p$ & $a_{p}(E)$ & $\mathbf{L}_{p}^{\text {imp }}\left(\mathrm{Sym}^{2} E, 1\right)^{\prime}$ & $\mathcal{L}_{p}^{\text {an }}\left(\mathrm{Sym}^{2} E\right)$ \\
\hline 5 & -2 & $p^{2}+O\left(p^{3}\right)$ & $4 p^{2}+O\left(p^{3}\right)$ \\
\hline 7 & 4 & $5 p+6 p^{2}+O\left(p^{3}\right)$ & $5 p+5 p^{2}+O\left(p^{3}\right)$ \\
\hline 13 & 6 & $5 p+O\left(p^{2}\right)$ & $10 p+O\left(p^{2}\right)$ \\
\hline
\end{tabular}

$E=264 \mathrm{~d} 1, C_{\mathrm{Sym}^{2} E}=132^{2}, S_{1}=\{2\}, \xi_{\mathrm{Sym}^{2} E}=2$

\begin{tabular}{|c|c|c|c|}
\hline$p$ & $a_{p}(E)$ & $\mathbf{L}_{p}^{\text {imp }}\left(\mathrm{Sym}^{2} E, 1\right)^{\prime}$ & $\mathcal{L}_{p}^{\text {an }}\left(\mathrm{Sym}^{2} E\right)$ \\
\hline 5 & 4 & $3 p+O\left(p^{2}\right)$ & $4+O(p)$ \\
\hline 7 & -2 & $6 p+O\left(p^{2}\right)$ & $4 p+O\left(p^{2}\right)$ \\
\hline
\end{tabular}

$E=268 \mathrm{a} 1, C_{\mathrm{Sym}^{2} E}=134^{2}, S_{1}=\{2\}, \xi_{\mathrm{Sym}^{2} E}=\frac{8}{3}$

\begin{tabular}{|c|c|c|c|}
\hline$p$ & $a_{p}(E)$ & $\mathbf{L}_{p}^{\mathrm{imp}}\left(\mathrm{Sym}^{2} E, 1\right)^{\prime}$ & $\mathcal{L}_{p}^{\text {an }}\left(\mathrm{Sym}^{2} E\right)$ \\
\hline 3 & 2 & $p^{-1}+2+p+O\left(p^{2}\right)$ & $p^{-1}+1+O\left(p^{2}\right)$ \\
\hline 5 & 2 & $4 p+3 p^{2}+O\left(p^{3}\right)$ & $2 p+4 p^{2}+O\left(p^{3}\right)$ \\
\hline 7 & 2 & $p+6 p^{2}+O\left(p^{3}\right)$ & $4 p+3 p^{2}+O\left(p^{3}\right)$ \\
\hline 11 & -4 & $3 p+O\left(p^{2}\right)$ & $10 p+O\left(p^{2}\right)$ \\
\hline 13 & -6 & $6 p+O\left(p^{2}\right)$ & $9 p+O\left(p^{2}\right)$ \\
\hline
\end{tabular}

$E=272 \mathrm{~d} 1, C_{\mathrm{Sym}^{2} E}=34^{2}, S_{1}=\{\}, \xi_{\mathrm{Sym}^{2} E}=\frac{1}{2}$

\begin{tabular}{|c|c|c|c|}
\hline$p$ & $a_{p}(E)$ & $\mathbf{L}_{p}^{\text {imp }}\left(\mathrm{Sym}^{2} E, 1\right)^{\prime}$ & $\mathcal{L}_{p}^{\text {an }}\left(\mathrm{Sym}^{2} E\right)$ \\
\hline 3 & 2 & $p+p^{2}+p^{3}+O\left(p^{4}\right)$ & $1+2 p+2 p^{2}+O\left(p^{3}\right)$ \\
\hline 7 & 4 & $3 p+2 p^{2}+O\left(p^{3}\right)$ & $5 p+p^{2}+O\left(p^{3}\right)$ \\
\hline 11 & -6 & $p+O\left(p^{2}\right)$ & $3 p+O\left(p^{2}\right)$ \\
\hline 13 & 2 & $7 p+O\left(p^{2}\right)$ & $10 p+O\left(p^{2}\right)$ \\
\hline
\end{tabular}

$E=280 \mathrm{a} 1, C_{\mathrm{Sym}^{2} E}=140^{2}, S_{1}=\{2\}, \xi_{\mathrm{Sym}^{2} E}=2$

\begin{tabular}{|c|c|c|c|}
\hline$p$ & $a_{p}(E)$ & $\mathbf{L}_{p}^{\text {imp }}\left(\mathrm{Sym}^{2} E, 1\right)^{\prime}$ & $\mathcal{L}_{p}^{\text {an }}\left(\mathrm{Sym}^{2} E\right)$ \\
\hline 3 & -1 & $p+2 p^{2}+2 p^{3}+O\left(p^{4}\right)$ & $2+p+p^{2}+O\left(p^{3}\right)$ \\
\hline 11 & -5 & $7 p+O\left(p^{2}\right)$ & $8 p+O\left(p^{2}\right)$ \\
\hline 13 & 1 & $O(p)$ & $O(1)$ \\
\hline
\end{tabular}

$E=280 \mathrm{~b} 1, C_{\mathrm{Sym}^{2} E}=140^{2}, S_{1}=\{2\}, \xi_{\mathrm{Sym}^{2} E}=2$

\begin{tabular}{|c|c|c|c|}
\hline$p$ & $a_{p}(E)$ & $\mathbf{L}_{p}^{\text {imp }}\left(\mathrm{Sym}^{2} E, 1\right)^{\prime}$ & $\mathcal{L}_{p}^{\text {an }}\left(\mathrm{Sym}^{2} E\right)$ \\
\hline 11 & -5 & $4 p+O\left(p^{2}\right)$ & $3 p+O\left(p^{2}\right)$ \\
\hline 13 & -5 & $10 p+O\left(p^{2}\right)$ & $9 p+O\left(p^{2}\right)$ \\
\hline
\end{tabular}

$E=288 \mathrm{a} 1, C_{\mathrm{Sym}^{2} E}=24^{2}, S_{1}=\{2,3\}, \xi_{\mathrm{Sym}^{2} E}=\frac{1}{2}$

\begin{tabular}{|c|c|c|c|}
\hline$p$ & $a_{p}(E)$ & $\mathbf{L}_{p}^{\text {imp }}\left(\mathrm{Sym}^{2} E, 1\right)^{\prime}$ & $\mathcal{L}_{p}^{\text {an }}\left(\mathrm{Sym}^{2} E\right)$ \\
\hline 5 & -4 & $2 p^{2}+O\left(p^{3}\right)$ & $4 p+O\left(p^{2}\right)$ \\
\hline 13 & -6 & $7 p+O\left(p^{2}\right)$ & $4 p+O\left(p^{2}\right)$ \\
\hline
\end{tabular}


$E=296 \mathrm{a} 1, C_{\mathrm{Sym}^{2} E}=148^{2}, S_{1}=\{2\}, \xi_{\mathrm{Sym}^{2} E}=2$

\begin{tabular}{|c|c|c|c|}
\hline$p$ & $a_{p}(E)$ & $\mathbf{L}_{p}^{\text {imp }}\left(\mathrm{Sym}^{2} E, 1\right)^{\prime}$ & $\mathcal{L}_{p}^{\mathrm{an}}\left(\mathrm{Sym}^{2} E\right)$ \\
\hline 3 & -1 & $p^{2}+O\left(p^{4}\right)$ & $2 p+O\left(p^{3}\right)$ \\
\hline 5 & -2 & $4 p+p^{2}+O\left(p^{3}\right)$ & $p+p^{2}+O\left(p^{3}\right)$ \\
\hline 7 & 1 & $4 p+O\left(p^{2}\right)$ & $6+O(p)$ \\
\hline 11 & 1 & $O(p)$ & $O(1)$ \\
\hline 13 & -6 & $2 p+O\left(p^{2}\right)$ & $4 p+O\left(p^{2}\right)$ \\
\hline
\end{tabular}

$E=296 \mathrm{~b} 1, C_{\mathrm{Sym}^{2} E}=148^{2}, S_{1}=\{2\}, \xi_{\mathrm{Sym}^{2} E}=2$

\begin{tabular}{|c|c|c|c|}
\hline$p$ & $a_{p}(E)$ & $\mathbf{L}_{p}^{\text {imp }}\left(\mathrm{Sym}^{2} E, 1\right)^{\prime}$ & $\mathcal{L}_{p}^{\text {an }}\left(\mathrm{Sym}^{2} E\right)$ \\
\hline 3 & -1 & $2 p+2 p^{2}+p^{3}+O\left(p^{4}\right)$ & $1+2 p+p^{2}+O\left(p^{3}\right)$ \\
\hline 7 & -3 & $2 p+3 p^{2}+O\left(p^{3}\right)$ & $2 p+5 p^{2}+O\left(p^{3}\right)$ \\
\hline 11 & -3 & $8 p+O\left(p^{2}\right)$ & $10 p+O\left(p^{2}\right)$ \\
\hline
\end{tabular}

$E=300 \mathrm{a} 1, C_{\mathrm{Sym}^{2} E}=30^{2}, S_{1}=\{2,5\}, \xi_{\mathrm{Sym}^{2} E}=\frac{8}{9}$

\begin{tabular}{|c|c|c|c|}
\hline$p$ & $a_{p}(E)$ & $\mathbf{L}_{p}^{\text {imp }}\left(\mathrm{Sym}^{2} E, 1\right)^{\prime}$ & $\mathcal{L}_{p}^{\text {an }}\left(\mathrm{Sym}^{2} E\right)$ \\
\hline 7 & 1 & $p+O\left(p^{2}\right)$ & $6+O(p)$ \\
\hline 11 & 6 & $5 p^{2}+O\left(p^{3}\right)$ & $5 p^{2}+O\left(p^{3}\right)$ \\
\hline 13 & -5 & $3 p+O\left(p^{2}\right)$ & $9 p+O\left(p^{2}\right)$ \\
\hline
\end{tabular}

\section{B.2 Tables of $\mathcal{L}$-invariants for elliptic curves $E$ with $D(E, 1)=0$}

Included below are the values we computed for both the derivative of $\mathbf{L}_{p}^{\text {aut }}\left(\operatorname{Sym}^{2} E, s\right)$ at $s=1$ and the corresponding $\mathcal{L}$-invariant term, for the six exceptional elliptic curves with $D(E, 1)=0$ (we omitted these specimens from $\S B .1$ as $\mathbf{L}_{p}^{\mathrm{imp}}\left(\mathrm{Sym}^{2} E, 1\right)^{\prime}=0$ for each of these six curves). To calculate these $p$-adic numbers, we used the generalised congruences given in Theorem 3.2 .

$E=176 \mathrm{~b} 1, C_{\mathrm{Sym}^{2} E}=11^{2}, S_{1}=\{2\}, \xi_{\mathrm{Sym}^{2} E}=\frac{2}{5}$
\begin{tabular}{|c|c|c|c|}
\hline$p$ & $a_{p}(E)$ & $\mathbf{L}_{p}^{\text {imp }}\left(\mathrm{Sym}^{2} E, 1\right)^{\prime}$ & $\mathcal{L}_{p}^{\text {an }}\left(\mathrm{Sym}^{2} E\right)$ \\
\hline 3 & 1 & $p+O\left(p^{4}\right)$ & $1+2 p^{2}+O\left(p^{3}\right)$ \\
\hline 5 & 1 & $p+O\left(p^{2}\right)$ & $p+O\left(p^{2}\right)$ \\
\hline 7 & 2 & $2 p+O\left(p^{2}\right)$ & $2 p+O\left(p^{2}\right)$ \\
\hline 13 & 4 & $4 p+O\left(p^{2}\right)$ & $2 p+O\left(p^{2}\right)$ \\
\hline
\end{tabular}

$E=196 \mathrm{a} 1, C_{\mathrm{Sym}^{2} E}=14^{2}, S_{1}=\{2,7\}, \xi_{\mathrm{Sym}^{2} E}=\frac{2}{9}$

\begin{tabular}{|c|c|c|c|}
\hline$p$ & $a_{p}(E)$ & $\mathbf{L}_{p}^{\mathrm{imp}}\left(\mathrm{Sym}^{2} E, 1\right)^{\prime}$ & $\mathcal{L}_{p}^{\mathrm{an}}\left(\mathrm{Sym}^{2} E\right)$ \\
\hline 3 & -1 & $1+2 p+p^{2}+O\left(p^{3}\right)$ & $2 p+p^{2}+2 p^{3}+O\left(p^{4}\right)$ \\
\hline 5 & -3 & $3 p+O\left(p^{2}\right)$ & $3 p+O\left(p^{2}\right)$ \\
\hline 11 & -3 & $p+O\left(p^{2}\right)$ & $3 p+O\left(p^{2}\right)$ \\
\hline 13 & -2 & $10 p+O\left(p^{2}\right)$ & $8 p+O\left(p^{2}\right)$ \\
\hline
\end{tabular}




$E=200 \mathrm{~b} 1, C_{\mathrm{Sym}^{2} E}=20^{2}, S_{1}=\{2,5\}, \xi_{\mathrm{Sym}^{2} E}=\frac{1}{2}$
\begin{tabular}{|c|c|c|c|}
\hline$p$ & $a_{p}(E)$ & $\mathbf{L}_{p}^{\text {imp }}\left(\mathrm{Sym}^{2} E, 1\right)^{\prime}$ & $\mathcal{L}_{p}^{\text {an }}\left(\mathrm{Sym}^{2} E\right)$ \\
\hline 3 & -2 & $p+p^{3}+O\left(p^{4}\right)$ & $1+p+p^{2}+O\left(p^{3}\right)$ \\
\hline 7 & -2 & $4 p+O\left(p^{2}\right)$ & $6 p+O\left(p^{2}\right)$ \\
\hline 11 & -4 & $7 p+O\left(p^{2}\right)$ & $p+O\left(p^{2}\right)$ \\
\hline 13 & -4 & $O\left(p^{2}\right)$ & $O\left(p^{2}\right)$ \\
\hline
\end{tabular}

$E=240 \mathrm{~d} 1, C_{\mathrm{Sym}^{2} E}=15^{2}, S_{1}=\{2\}, \xi_{\mathrm{Sym}^{2} E}=\frac{1}{4}$

\begin{tabular}{|c|c|c|c|}
\hline$p$ & $a_{p}(E)$ & $\mathbf{L}_{p}^{\text {imp }}\left(\mathrm{Sym}^{2} E, 1\right)^{\prime}$ & $\mathcal{L}_{p}^{\text {an }}\left(\mathrm{Sym}^{2} E\right)$ \\
\hline 11 & 4 & $10 p+O\left(p^{2}\right)$ & $6 p+O\left(p^{2}\right)$ \\
\hline 13 & -2 & $3 p+O\left(p^{2}\right)$ & $3 p+O\left(p^{2}\right)$ \\
\hline
\end{tabular}

$E=272 \mathrm{~b} 1, C_{\mathrm{Sym}^{2} E}=17^{2}, S_{1}=\{2\}, \xi_{\mathrm{Sym}^{2} E}=\frac{1}{4}$

\begin{tabular}{|c|c|c|c|}
\hline$p$ & $a_{p}(E)$ & $\mathbf{L}_{p}^{\text {imp }}\left(\mathrm{Sym}^{2} E, 1\right)^{\prime}$ & $\mathcal{L}_{p}^{\mathrm{an}}\left(\mathrm{Sym}^{2} E\right)$ \\
\hline 5 & -2 & $p+4 p^{2}+O\left(p^{3}\right)$ & $2 p+2 p^{2}+O\left(p^{3}\right)$ \\
\hline 7 & -4 & $2 p+O\left(p^{2}\right)$ & $2 p+O\left(p^{2}\right)$ \\
\hline 13 & -2 & $O(p)$ & $O(p)$ \\
\hline
\end{tabular}

$E=300 \mathrm{c} 1, C_{\mathrm{Sym}^{2} E}=30^{2}, S_{1}=\{2,5\}, \xi_{\mathrm{Sym}^{2} E}=\frac{1}{3}$

\begin{tabular}{|c|c|c|c|}
\hline$p$ & $a_{p}(E)$ & $\mathbf{L}_{p}^{\text {imp }}\left(\mathrm{Sym}^{2} E, 1\right)^{\prime}$ & $\mathcal{L}_{p}^{\text {an }}\left(\mathrm{Sym}^{2} E\right)$ \\
\hline 7 & 4 & $2 p+O\left(p^{2}\right)$ & $5 p+O\left(p^{2}\right)$ \\
\hline 11 & -4 & $4 p+O\left(p^{2}\right)$ & $4 p+O\left(p^{2}\right)$ \\
\hline
\end{tabular}

\section{References}

[1] K. Barré-Sireix, G. Diaz, F. Gramain and G. Philibert, Une preuve de la conjecture MahlerManin, Invent. Math. 124 (1996), 1-9.

[2] D. Benois, A generalization of Greenberg's $\mathcal{L}$-invariant, American Journal of Mathematics 133 (2011), 1573-1632.

[3] S. Bloch and K. Kato, L-functions and Tamagawa numbers of motives, in the Grothendieck Festschrift I, Progress in Math., Birkäuser, Boston (1990), 333-400.

[4] C. Breuil, B. Conrad, F. Diamond and R. Taylor, On the modularity of elliptic curves over $\mathbb{Q}$ : wild 3-adic exercises, Journal of the American Math. Soc. 14 (2001), 843-939.

[5] C. Citro, $\mathcal{L}$-invariants of adjoint square Galois representations coming from modular forms, Int. Math. Res. Notices no. 14 (2008), Art. ID rnn048.

[6] J. Coates and B. Perrin-Riou, On p-adic L-functions attached to motives over $\mathbb{Q}$, Advanced Studies in Pure Mathematics 17, Academic Press, Boston (1989), 23-54.

[7] J. Coates and C.-G. Schmidt, Iwasawa theory for the symmetric square of an elliptic curve, Journal Reine Angew. Math. 375/376 (1987), 104-156. 
[8] J. Cremona, Elliptic curve data, available at http://johncremona.github.io/ecdata/

[9] A. Dabrowski and D. Delbourgo, S-adic L-functions attached to the symmetric square of a newform, Proc. of the London Math. Soc. 74 (1997), 559-611.

[10] S. Dasgupta, Factorization of p-adic Rankin series, Invent. Math. 205 (2016), 221-268.

[11] D. Delbourgo, $\mathcal{L}$-invariants arising from p-adic measures of $S^{2} m^{2} E$, Glasgow Mathematical Journal 44 (2001), 45-64.

[12] F. Diamond, On deformation rings and Hecke rings, Annals of Mathematics 144 (1996), $137-166$.

[13] E. Dummit, M. Hablicsek, R. Harron, L. Jain, R. Pollack and D. Ross, Explicit computations of Hida families via overconvergent modular symbols, Research in Number Theory 2:25 (2016), DOI: 10.1007/s40993-016-0052-8

[14] B. Ferrero and R. Greenberg, On the behaviour of p-adic L-functions at $s=0$, Invent. Math. 50 (1978), 91-102.

[15] M. Flach, On the degree of modular parametrizations, Séminaire de Théorie des Nombres, Paris 1991/92, Progr. Math. 116, Birkhuser Boston, 23-36.

[16] M. Flach, A finiteness theorem for the symmetric square of an elliptic curve, Invent. Math. 109 (1992), 307-327.

[17] S. Gelbart and H. Jacquet, A relation between automorphic L-functions of $G L(2)$ and GL(3), Ann. Sci. École Norm. Sup. 11 (1978), 471-542.

[18] B. Gorsse and G. Robert, Appendix to Mesures p-adiques associées aux carrés symétriques, Thèse de Doctorat, Université de Grenoble I (2006).

[19] R. Greenberg, Trivial zeros of p-adic L-functions, in " $p$-adic monodromy and the Birch and Swinnerton-Dyer conjecture", Contemp. Math. 165 (1991), 149-181.

[20] B. Gross and D. Zagier, Heegner points and derivatives of L-series, Invent. Math. 84 (1984), $225-320$.

[21] P. Guerzhoy, Jacobi-Eisenstein series and p-adic interpolation of symmetric squares of cusp forms, Annales Inst. Fourier 45 (1995), 605-625.

[22] R. Harron and A. Lei, Iwasawa theory for symmetric powers of CM modular forms at non-ordinary primes, Journal de Théorie des Nombres de Bordeaux 26 (2014), 673-707.

[23] H. Hida, A p-adic measure attached to the zeta functions associated with two elliptic modular forms II, Ann. Inst. Fourier 38 (1988), 1-83.

[24] H. Hida, p-adic L-functions for base change lifts of $G L_{2}$ to $G L_{3}$, in Automorphic Forms, Shimura Varieties and L-functions, Academic Press, San Diego (1990), 93-142.

[25] H. Hida, Adjoint Selmer groups as Iwasawa modules, Israel Journal of Math. 120 (2000), $361-427$.

[26] H. Hida, $\mathcal{L}$-invariants of adjoint square Galois representations, Int. Math. Res. Notices 59 (2004), 3177-3189. 
[27] D. Loeffler and S. Zerbes, Iwasawa theory for the symmetric square of a modular form, to appear in Journal Reine Angew. Math.

[28] T. Miyake, Modular forms, Springer Monographs in Mathematics, Springer-Verlag, Berlin (1989), 338 pages.

[29] M. Nastasescu, Determination of elliptic curves by their adjoint p-adic L-functions, Journal of Number Theory 152 (2015), 156-181.

[30] G. Rosso, A formula for the derivative of the p-adic L-function of the symmetric square of a finite slope modular form, Amer. J. Math. 138 (2016), 821-878.

[31] C.-G. Schmidt, p-adic measures attached to automorphic representations of $G L_{3}$, Invent. Math. 92 (1988), 597-631.

[32] J.-P. Serre, Propriétés galoisiennes des points d'ordre finis des courbes elliptiques, Invent. Math. 15 (1972), 259-331.

[33] G. Shimura, The special values of the zeta functions associated with cusp forms, Com. Pure and Appl. Math. 29 (1976), 783-804.

[34] W. Stein, Modular forms, a computational approach, Graduate Studies in Mathematics, 79 (2007), 268 pages.

[35] A. Wiles, Modular elliptic curves and Fermat's last theorem, Annals of Mathematics 141 (1995), 443-551. 